\title{
WestVirginiaUniversity
}

THE RESEARCH REPOSITORY @ WVU

Graduate Theses, Dissertations, and Problem Reports

2005

\section{Optical detection of multiple faults of a MEMS based linear comb resonator}

Lakshmi Deepasree Konduparthi

West Virginia University

Follow this and additional works at: https://researchrepository.wvu.edu/etd

\section{Recommended Citation}

Konduparthi, Lakshmi Deepasree, "Optical detection of multiple faults of a MEMS based linear comb resonator" (2005). Graduate Theses, Dissertations, and Problem Reports. 1671.

https://researchrepository.wvu.edu/etd/1671

This Thesis is protected by copyright and/or related rights. It has been brought to you by the The Research Repository @ WVU with permission from the rights-holder(s). You are free to use this Thesis in any way that is permitted by the copyright and related rights legislation that applies to your use. For other uses you must obtain permission from the rights-holder(s) directly, unless additional rights are indicated by a Creative Commons license in the record and/ or on the work itself. This Thesis has been accepted for inclusion in WVU Graduate Theses, Dissertations, and Problem Reports collection by an authorized administrator of The Research Repository @ WVU. For more information, please contact researchrepository@mail.wvu.edu. 


\title{
Optical Detection of Multiple Faults of a MEMS Based Linear Comb Resonator
}

\author{
Lakshmi Deepasree Konduparthi \\ Thesis submitted to the \\ College of Engineering and Mineral Resources at \\ West Virginia University \\ in partial fulfillment of the requirements \\ for the degree of
}

MASTER OF SCIENCE

in

ELECTRICAL ENGINEERING

Dr. Lawrence Hornak, Ph.D., Chair

Dr.Parviz Famouri, Ph.D.

Dr. Dimitris Korakakis, Ph.D.

Lane Department of Computer Science and Electrical Engineering

West Virginia University

Morgantown, West Virginia

2005

Keywords: Fault Detection,MEMS, Reliability, Optical Monitoring 


\section{ABSTRACT \\ OPTICAL DETECTION OF MULTIPLE FAULTS ON A MEMS BASED LINEAR COMB RESONATOR}

\section{LAKSHMI DEEPASREE KONDUPARTHI}

Investigating the dynamic behavior of any Micro Electro Mechanical system is critical for better control and fault prediction. Linear Comb Resonator is one such MEMS generic device that exhibits motion parallel to the plane of the chip. The dynamic motion of the Linear Comb Resonator is characterized by various parameters such as mass, force applied and also the stiffness constant of the flexure that holds the device. The aim of the project is to determine the changes caused by the asymmetry in the flexure design and the fluctuations in the behavior of the resonator due to this. The changes in the stiffness constant, resonant frequency and the motion were simulated based on the equations in a previous study. But this theoretical understanding was not sufficient to detect the asymmetries in the device. To understand the effects due to asymmetry, a finite element solid model was developed and analyzed using ANSYS software. Successful validation of the model was done and the same was used to determine the behavior of the model under various defects. In a real time system, more than one defect occur at the same time, and therefore, the effect of multiple faults on the flexures of the device was analyzed and the same changes were incorporated in the die design to verify the behavior experimentally. The difference in the displacements at the ends of the flexures was found to be a good parameter to distinguish between the defective and defect free devices. Optical signal obtained at the two ends of the flexures, is shown to be sufficient to determine defective devices. Such a thorough analysis of the anomalies due to this asymmetry of the flexure structure gives us a better understanding about the reliability of the system and also the ability to anticipate and detect faults. 
To My Father 


\section{Acknowledgments}

I would like to thank my committee members, Dr. Larry Hornak, Dr. Parviz Famouri, and Dr. Dimitris Korakakis. I would like to specially thank Dr.Hornak, my research advisor, for his guidance, patience and support throughout the project. I would also like to thank Dr. Nilay Mukherjee for guiding me through the validation process. I thank Dr.Kolin Brown for his training and constant inputs. I also thank NASA Epscor for funding this project

Additionally, I thank members of MEMS research group Afshin Izadian, Limin Wang, William McCormick and Scott Rittenhouse for their help and training in understanding the project. I also would like to thank my colleagues in MSRC for their encouragement.

Above all, I thank my parents for motivating and inspiring me to pursue an advanced degree. I also thank my sister for her love and encouragement. In addition I thank my friends and roomates. 


\section{Contents}

$\begin{array}{ll}\text { Dedication } & \text { iii }\end{array}$

$\begin{array}{ll}\text { Abstract } & \text { ii }\end{array}$

Acknowledgements $\quad$ iv

$\begin{array}{lll}\text { Contents } & \mathbf{v}\end{array}$

List of Figures $\quad$ viii

List of Tables $\quad$ xi

1 Introduction 1

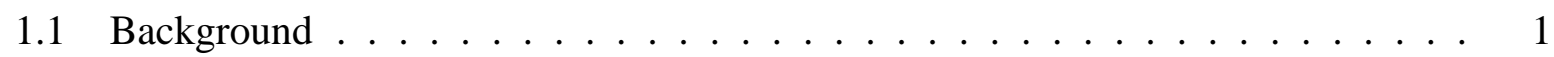

1.2 Measurement Techniques . . . . . . . . . . . . . . . . . . 2

1.3 Reliability .......................... 5

1.3.1 Fault detection and prediction in MEMS . . . . . . . . . . . 5

1.4 Organization of Thesis $\ldots \ldots \ldots \ldots \ldots \ldots$

2 Theory and validation $\quad 8$

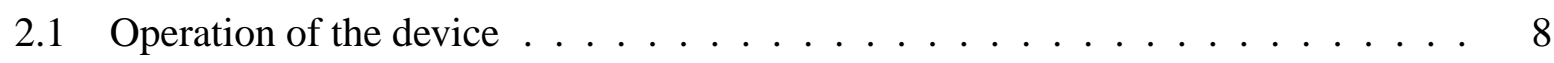

2.2 Structural Mechanics . . . . . . . . . . . . . . . . . 10

2.2.1 Elasticity Theory . . . . . . . . . . . . . . . . 11

2.2.2 Finite Element Analysis . . . . . . . . . . . . . . . . 12 


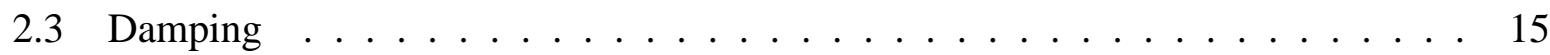

3 MEMS sensitivity analysis and validation $\quad 18$

3.1 Development of solid model . . . . . . . . . . . . . . . 18

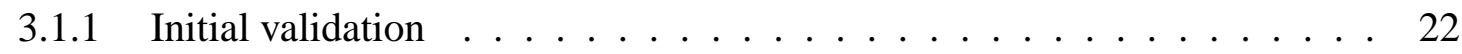

3.1 .2 Residual Stress . . . . . . . . . . . . . . 23

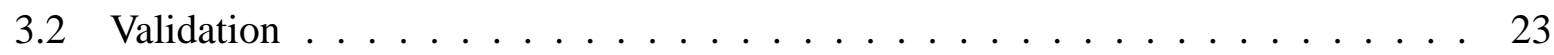

3.3 Fabrication Tolerance . . . . . . . . . . . . . . . 25

3.3 .1 Sensitivity analysis . . . . . . . . . . . . 26

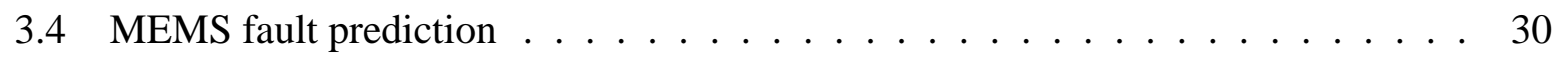

4 MEMS Device design and testing $\quad 40$

4.1 MEMS Layout Design . . . . . . . . . . . . . . . . . . . . . 40

4.2 Processing of the MEMS Die . . . . . . . . . . . . . . . 48

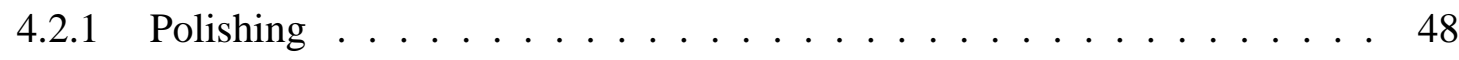

4.2 .2 Release ............................ 48

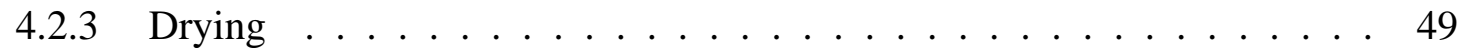

4.2 .4 Fabrication of glass packages . . . . . . . . . . . . . . 49

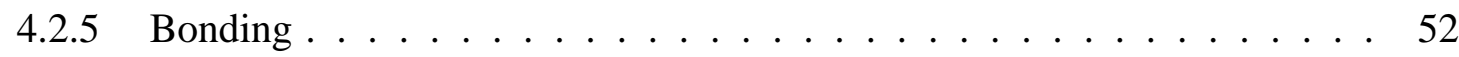

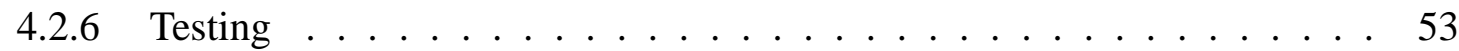

5 Experimental Results and Discussion $\quad 54$

5.1 Optical Testing and Setup . . . . . . . . . . . . . . 55

5.2 Assumptions and recovery of optical data . . . . . . . . . . . . 56

5.3 Initial Results . . . . . . . . . . . . . . . . . . . . . 60

5.4 Fault Detection $\ldots \ldots \ldots \ldots \ldots \ldots \ldots \ldots$

6 Conclusions and Future Work $\quad 71$

6.1 Future Work . . . . . . . . . . . . . . . . . 72 
A ANSYS Codes

B MATLAB Codes 


\section{List of Figures}

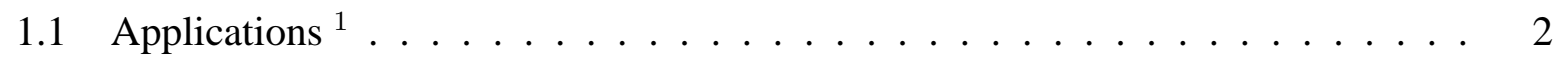

1.2 Cross section of layers used in the MUMPS process . . . . . . . . . . . . 3

1.3 Applications ........................... 4

2.1 Features of LCR . . . . . . . . . . . . . . . . . . 9

2.2 Lumped model of the LCR . . . . . . . . . . . . . . . . . 11

2.3 Stresses on a Solid Body . . . . . . . . . . . . . . . . . 12

3.1 Contours . . . . . . . . . . . . . . . . . . . . . . 19

3.2 Contours . . . . . . . . . . . . . . . . . . 20

3.3 Meshed Model . . . . . . . . . . . . . . . . . . . . . 21

3.4 Theory and Ansys comparison . . . . . . . . . . . . . . 22

3.5 Residual Stress: Displacement Plots . . . . . . . . . . . . . . . . . 23

3.6 Frequency response when damping was ignored . . . . . . . . . . . . . . 24

3.7 ANSYS and Experimental comparison . . . . . . . . . . . . . . 25

3.8 Fabrication Tolerance . . . . . . . . . . . . . . . . . . . 27

3.9 Parameters analyzed . . . . . . . . . . . . . . . 27

3.10 Variation in resonant frequency with Length of beam and Width of the beam . . . 28

3.11 Variation in resonant frequency with Length of beam and Width of the truss . . . 29

3.12 Beam Classification . . . . . . . . . . . . . . . . . . . . . 30

3.13 Resonant frequency variation with the change in width of a single beam . . . . 31

3.14 Peak amplitude variation with the change in width of a single beam . . . . . . . . 32 
3.15 Resonant frequency variation due to multiple faults on anchor beam . . . . . . . 34

3.16 Resonant frequency variation due to multiple faults on corner beam . . . . . . 35

3.17 Difference in displacements at the ends of the flexure with variation of anchor beam width $\ldots \ldots \ldots \ldots \ldots \ldots$

3.18 Difference in displacements at the ends of the flexure with variation of corner beam width . . . . . . . . . . . . . . . . . . . 37

3.19 Difference in displacements at the ends of the flexure under multiple faults:Anchor

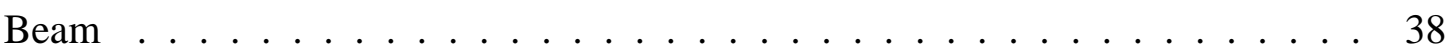

3.20 Difference in displacements at the ends of the flexure under multiple faults:Corner

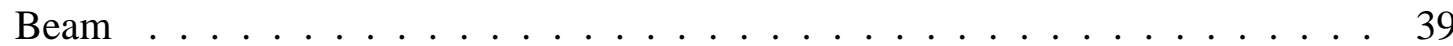

4.1 Final Die Layout $\ldots \ldots \ldots \ldots$. . . . . . . . . . . . . . . . 43

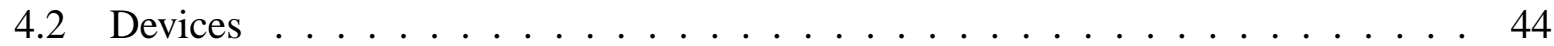

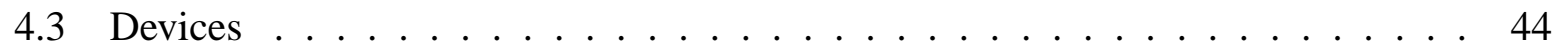

4.4 Multiple Faults . . . . . . . . . . . . . . . . . . . . . . . 45

4.5 Multiple Faults . . . . . . . . . . . . . . . . . . . . . 45

4.6 Resonant frequency variation due to multiple faults on anchor beam of a $3 \mu \mathrm{m}$ beam width device . . . . . . . . . . . . . . . . . . . . . 46

4.7 Resonant frequency variation due to multiple faults on corner beam of a $3 \mu \mathrm{m}$ beam width device . . . . . . . . . . . . . . . . . . . . . 47

4.8 Difference in displacements at the ends of the flexure under multiple faults of a $3 \mu m$ beam width device:Anchor Beam . . . . . . . . . . . . . . . 47

4.9 Difference in displacements at the ends of the flexure under multiple faults of a $3 \mu m$ beam width device:Corner Beam . . . . . . . . . . . . . . . . 48

4.10 SEM Pictures $\ldots \ldots \ldots \ldots$. . . . . . . . . . . . . . . . . 49

4.11 SEM Pictures $\ldots \ldots \ldots \ldots \ldots$

4.12 SEM Pictures $\ldots \ldots \ldots \ldots \ldots \ldots$

4.13 SEM Pictures . . . . . . . . . . . . . . . . . . . . 51

4.14 SEM Pictures $\ldots \ldots \ldots \ldots \ldots \ldots \ldots \ldots \ldots$ 
4.15 Fabricated Gold Package . . . . . . . . . . . . . . . . . 52

4.16 Bonded Die . . . . . . . . . . . . . . . . . . . . 53

5.1 Basic Operation of the vibrometer ${ }^{1} \ldots \ldots \ldots \ldots$

5.2 Optical signal from the vibrometer . . . . . . . . . . . . . 55

5.3 The placement of measurement and reference beams on the device . . . . . . . 56

5.4 Experimental Setup . . . . . . . . . . . . . . . . . . . . 57

5.5 Simulation Validation $\ldots \ldots \ldots \ldots \ldots \ldots$

5.6 Optical signal comparison at the ends of a defect free device . . . . . . . . . . 59

5.7 Optical signal comparison at the ends of a defective device . . . . . . . . . 60

5.8 Frequency response of the T450 Device . . . . . . . . . . . . . 61

5.9 Resonant frequencies of defective devices . . . . . . . . . . . . . . . 62

5.10 Optical signal values at the stage of a defect free and defective device . . . . . 62

5.11 Recovered frequency response comparison of defective and defect free devices . 63

5.12 Frequency response envelope . . . . . . . . . . . . . . . . . 63

5.13 Anchor beam Multiple fault detection . . . . . . . . . . . . . . . 64

5.14 Recovered displacement of a $20 \%$ point of adhesion device . . . . . . . . . 65

5.15 Recovered displacement of a 50\% point of adhesion device . . . . . . . . . 66

5.16 Recovered displacement of an over-etched 50\% point of adhesion device . . . . . 67

5.17 Optical detection of Faults:Anchor Beam $\ldots \ldots \ldots$. . . . . . . . . 67

5.18 Corner beam multi fault detection $\ldots \ldots \ldots$. . . . . . . . . 68

5.19 Recovered displacement of an over-etched $10 \%$ point of adhesion device:Corner

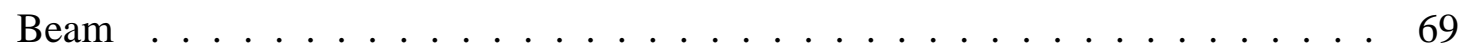

5.20 Optical detection of Faults:Corner Beam ～. . . . . . . . . . . . . . 69

5.21 Recovered displacement of a $10 \%$ point of adhesion device:Corner Beam . . . 70 


\section{List of Tables}

1.1 Thickness of the MUMPS layers . . . . . . . . . . . . . . 3

3.1 Summary of elements used . . . . . . . . . . . . . . . . 21

3.2 Theory and Ansys comparison . . . . . . . . . . . . . . . 22

3.3 ANSYS and Experimental data comparison . . . . . . . . . . . . 26

3.4 Range of resonant frequency within the fabrication tolerances . . . . . . . . . 26

3.5 Percentage change in resonant frequency . . . . . . . . . . . . . . 29

3.6 Resonant frequency variation with change of single beam width . . . . . . . . 31

3.7 Amplitude variation with change of single beam width . . . . . . . . . 33

3.8 Particles under various parts of the device $\ldots \ldots \ldots \ldots$

3.9 Difference in displacement at ends of flexures with anchor beam width change . 37

3.10 Difference in displacement at ends of flexures with Corner beam width change . 38

4.1 Devices in the present layout . . . . . . . . . . . . . 41

4.2 Range of parameters due to fabrication tolerance: Current Die . . . . . . . . 42

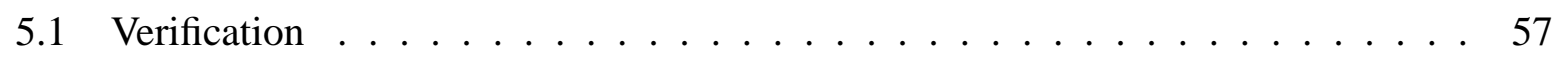

5.2 Standard Deviation . . . . . . . . . . . . . . . . 58 


\section{Chapter 1}

\section{Introduction}

\subsection{Background}

Micro Electro Mechanical Systems(MEMS) are composed of micro mechanical structures which can be electrically actuated. These micro structures can be used as sensors or actuators, with broad applications. They are used in safety critical systems like Air-Bags, Drug Delivery Systems, and Ice Sensors [41, 24]. Sensors are used in accelerometers, gyroscopes, pressure gauges, capacitance sensors, ink-jet printers etc. As an actuator they are used in applications including drive gears (Sandia Labs and Fig.1.1a), Drug delivery systems [24], and lens actuators for imaging purposes [16]. Fig.1.1a shows a micro gear designed at the Sandia Laboratories.

MEMS fabrication techniques mirror semiconductor technology and use similar growth techniques. MEMS are also fabricated using various techniques such as LIGA (which utilizes photolithography electroplating [9]), bulk micro machining and surface micro machining. LIGA is promising but requires further research before use in commercial applications [9]. Bulk micromachining involves etching of the substrate, generally silicon, to achieve the desired pattern, however poor sidewall definition and aggressive etching of large amounts of the substrate [2] hinder its integration with circuitry. Surface micromachining is one of the commonly used techniques for the growth of MEMS structures. This growth is done by thin film technology and patterned with photolithographic and dry etch techniques [2]. 


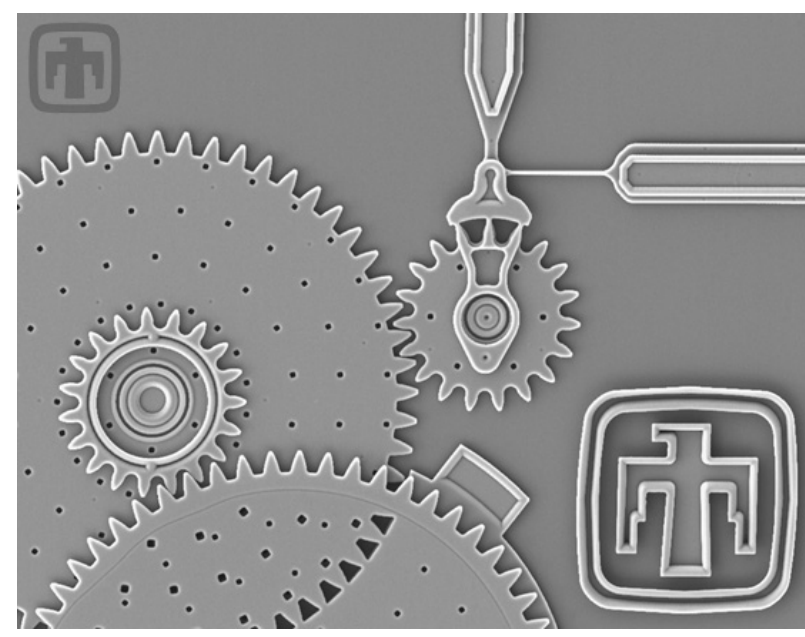

(a) Gear Transmission

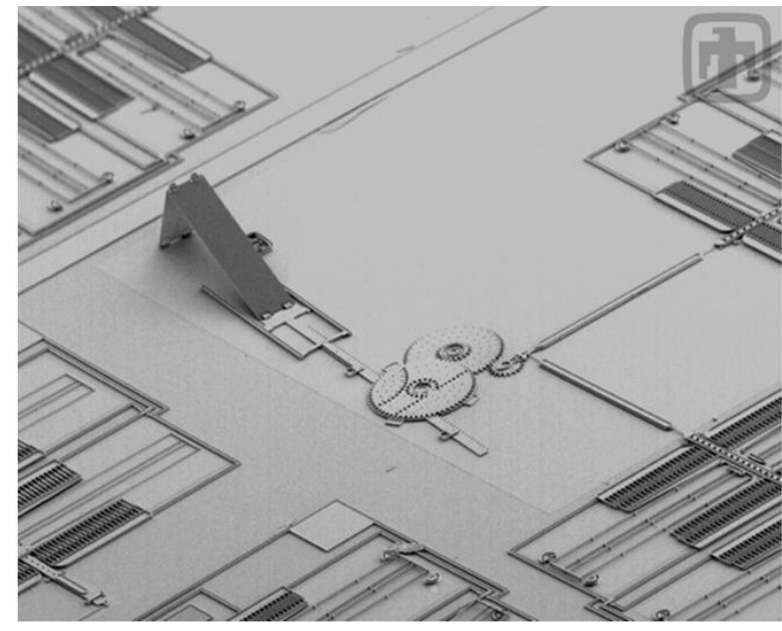

(b) Optical Mirror

Figure 1.1: Applications ${ }^{1}$

MEMS are manufactured using mainly, Silicon On Insulator (SOI), Metal MEMS technology or the poly MUMPs (Multi-User-MEMS-Processes) technology. For this work, Poly MUMPS fabricated at the MEMSCAP Corporation is used. These MEMS structures are made of 3 layers of polycrystalline silicon, otherwise known as poly silicon or just poly.

The principle of poly MUMPs is based on the mechanical properties of poly, which is stronger than steel and weighs only one third the weight [8]. This gives the ability to design very light and strong structures. The poly mumps process has silicon as the base substrate. Fig.1.2 shows the different layers used in the MUMPS fabrication process. Table 1.1 gives the typical thin film thicknesses. Basic structures are pattered in the poly1 layer; mass changes are made on the poly2 layer; poly0 is used as a foundation (and electrical ground plane) layer.

\subsection{Measurement Techniques}

Our current research at the West Virginia University involves the study of Linear Comb Resonator (LCR), a generic MEMS device for actuation and sensing. The MEMS LCR contains

\footnotetext{
${ }^{1}$ Sandia.gov Image Gallery
} 


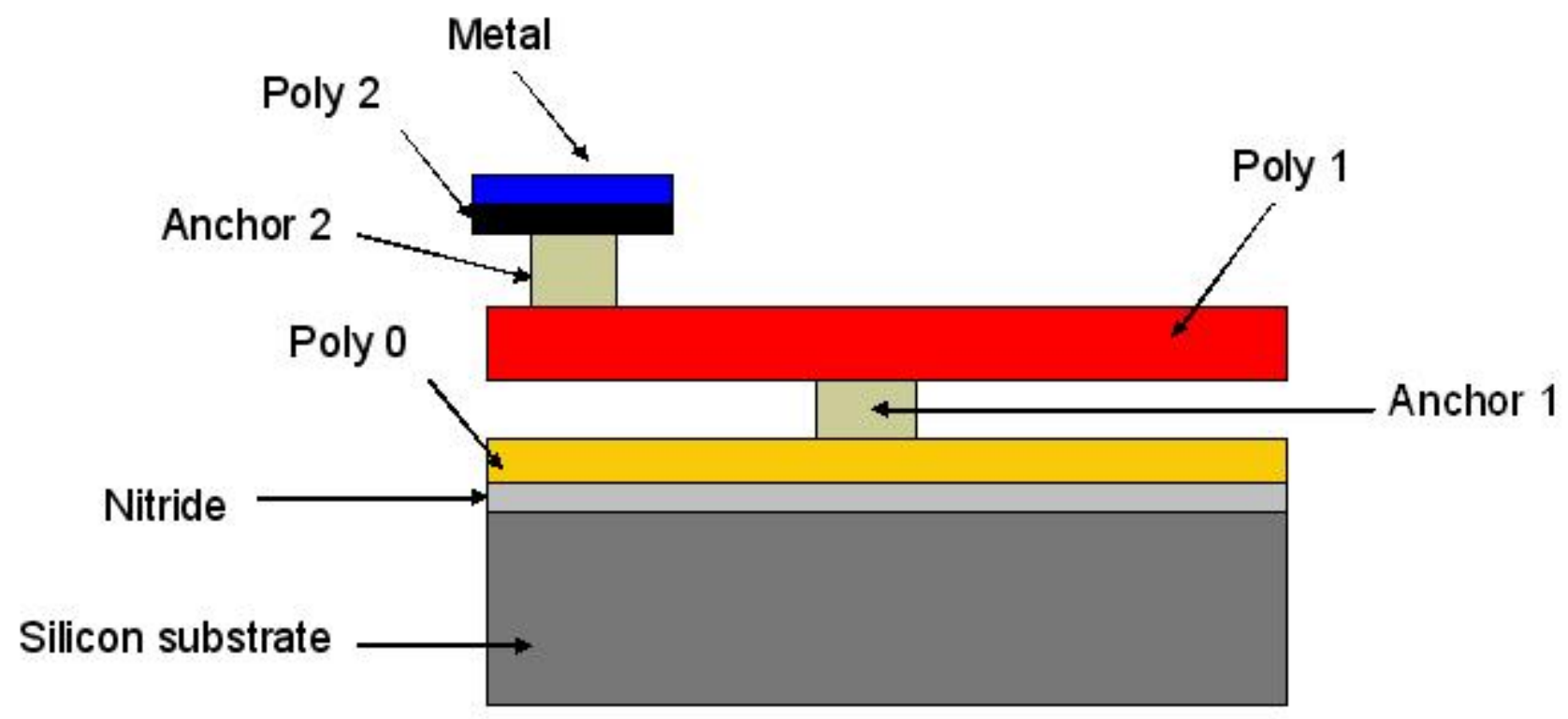

Figure 1.2: Cross section of layers used in the MUMPS process

\begin{tabular}{|c|c|c|}
\hline Layer Name & Material & Thickness \\
\hline Nitride & Silicon Nitride & $600 \mathrm{~nm}$ \\
Poly 0 & Polysilicon & $500 \mathrm{~nm}$ \\
Oxide 1 & Phosphosilicate glass & $2 \mu \mathrm{m}$ \\
Poly 1 & Polysilicon & $2 \mu \mathrm{m}$ \\
Oxide 2 & Phosphosilicate glass & $750 \mathrm{~nm}$ \\
Poly 2 & Polysilicon & $1.5 \mu \mathrm{m}$ \\
Metal & Gold & $500 \mathrm{~nm}$ \\
\hline
\end{tabular}

Table 1.1: Thickness of the MUMPS layers 


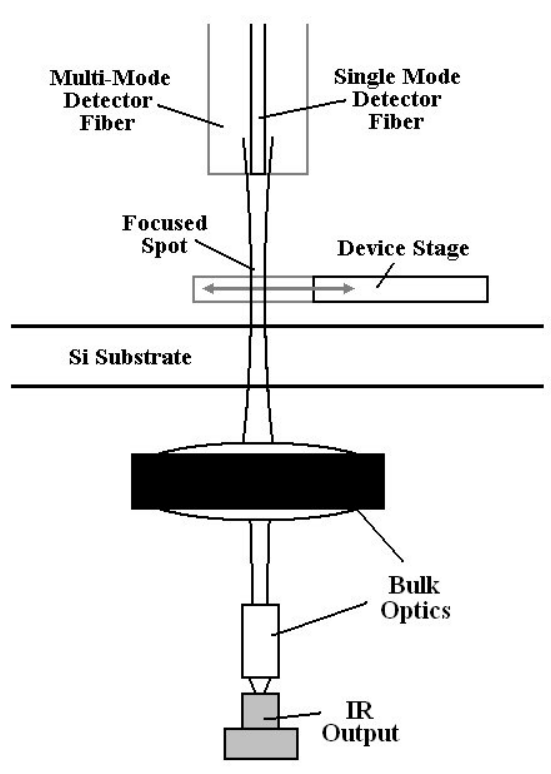

(a) Through wafer system

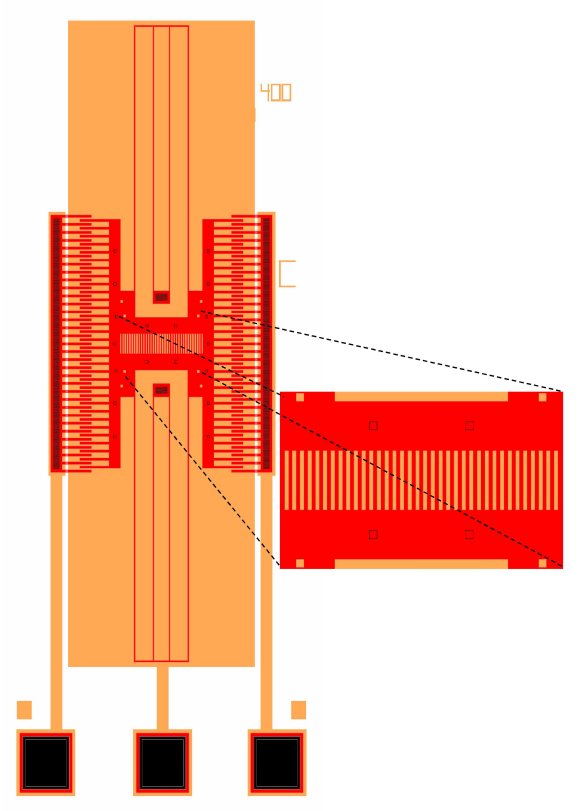

(b) Comb resonator and the gratings

Figure 1.3: Applications

all the basic components of an electrostatically driven MEMS device, including spring beam flexures, interdigitated moving and fixed fingers with designed electrostatic air gap and moving shuttle mass. Measurement of the mass position in this work is done optically using a near infrared through wafer setup as explained in [12]. A 1310nm wavelength laser is used, as silicon is transparent to this wavelength. The light is passed through the wafer as shown in Fig.1.3(a) and the transmitted beam, modulated by the motion of the moving shuttle mass, is detected by the optical fiber.

The MEMS LCR test device is designed to have $2 \mu \mathrm{m}$ grating structure as shown in Fig.1.3 (b). The light intensity gives a peak and valley when passing through an opening and space of the grating, thus giving information about the total displacement of the system. An autorecovery control block [38], is used to recover the displacement of the shuttle from this optical data. An optical system enables a completely decoupled measurement from the electrostatic drive [19], giving accurate results. 


\subsection{Reliability}

MEMS are increasingly being used in safety critical systems. The reliability of these devices is hence of vital importance. The more robust and reliable a device is, the better its commercial value. A defect is anything that affects its normal expected operation. Defects occur during the fabrication and operation of the MEMS devices due to factors like humidity, temperature, improper etching and other environmental conditions. The most common defects that MEMS devices face during their manufacturing and life time of operation are:

- Stiction: When the MEMS devices are "released", i.e. the oxide layer lying in between the poly 1 and poly 0 is etched away; the stiction forces due to the liquids used in the process pull down the structures.

- Particulate faults: This is the most common fault [22]. These occur due to dust, humidity, patterning of the structures during the manufacture of the devices and also during the operation. Particulates can occur on top of the poly 1 layer or in between poly 1 and poly 0 . They could also occur due to improperly released structures.

- Etch variations: The MEMSCAP surface micromaching has an etch tolerance limit of \pm $0.3 \mu \mathrm{m}$. These etch variations could be local or global. They can lead to unexpected fracture or premature fatigue.

- Package tilt: After packaging, due to various surface forces, the MEMS devices may tilt out of plane [15]. Due to their mechanical nature they also experience stresses, fracture etc. as a result of the same. Research efforts to minimize the residual stresses during the fabrication, in order to increase their life are ongoing [15].

\subsubsection{Fault detection and prediction in MEMS}

Fault detection or defect detection has been under study for a long time. As the reliability of a MEMS device is affected by various defects and faults, fault detection is of great importance. This can be done using many methods such as mathematical modeling, built in tests, external 
stimuli etc. Testing is done by checking a parameter or a change in the parameter of the device. A parameter could be any measurable or computable quantity such as mass, spring constant, damping coefficient, resonant frequency or the displacement of the device.

Fault detection is a challenge because of defect masking. Due to fabrication limitations and contaminations, the devices behave differently from the expected ideal response. For example, a defect free device, having a global variation due to a fabrication error and a defective device having a change in mass due to particulate contamination might have a parameter of interest in the same range. Hence parameter changes cannot be used to predict these faults. If a fault doesn't reflect in the parameters used in the mathematical modeling, then a much more comprehensive model needs to be developed.

Finite element analysis is being increasingly used in the characterization of MEMS. It is used for design, stress analysis, fracture and fatigue analysis etc [26, 36, 29, 35]. It is also now being used for fault analysis $[3,10,30,31]$. It gives an advantage because any non ideal behavior can be modeled easily once the development of the initial model is done successfully. Thus a finite element model gives a thorough understanding of the system's behavior under various faults.

One of the major challenges in fault detection is the determination of faults on the system. Built in self tests have been used by many researchers $[28,6]$. Most of the built in self test systems require that the system be taken off-line, before performing the test. This is not an ideal situation, especially if a system is used in a safety critical application. Non-obstructive fault detection system enables greater stability and reliability [30]. Also, in a real time situation a device is effected by more than single defect. In [13], Blanton et.al, have analyzed this situation for an accelerometer. They individually excite each of the stators electrically and compare the difference in two sides to detect any asymmetries. This kind of electrical excitation is not possible in the case of LCR. Therefore we need to look at a fault detection and testing scheme that would enable in-situ life time monitoring of the system through non-obstructive methods.

In this thesis, it is planned to show that finite element modeling can be used to determine the effects of multiple faults and that it is possible to detect such faults by monitoring the displacements at the ends of the flexures. The asymmetrical motion of the flexures would only be 
present when there is a local fault. It is further shown to distinguish the defect free device with a global variation and a defective device with a local variation. Also these defects can be monitored by optical probing of the ends of the flexures. Due to asymmetrical motion these are shown to give a different modulated signal on both ends, thus enabling decoupled optical fault detection. Here, devices which have multiple faults are designed and it is proved that these defects can be experimentally detected through optical monitoring.

\subsection{Organization of Thesis}

The theory and the various concepts used in the modeling of the design of the MEMS devices is given in Chapter 2. MEMS model development, simulation results and fault prediction results are described in Chapter 3. Design of MEMS die layout and the experimental procedure followed, is explained in Chapter 4. Experimental Results are presented in Chapter 5. This thesis is concluded with suggestions for future work in Chapter 6. 


\section{Chapter 2}

\section{Theory and validation}

In this chapter, the background theory required to understand various aspects of this thesis is presented. Section 2.1 talks about the basic operation of the device. Section 2.2 deals with the principles of elasticity theory and finite element theory, which are used to model the MEMS device under consideration. Section 2.3 describes the damping that the devices experience when they are subjected to movement.

\subsection{Operation of the device}

LCR is actuated due to the electrostatic attraction between stators and the stage[shuttle]. The Fig.2.1 shows the various parts of the resonator. The device behaves as a damped oscillator whose motion is governed by the equation 2.1

$$
F_{e}=m \ddot{x}+\beta \dot{x}+k x+F_{l}
$$

where $F_{e}$ is the electrostatic force, $\mathrm{m}$ is the effective mass, $\beta$ is the damping due to the surrounding medium, $\mathrm{k}$ is the effective spring constant. All these parameters are direction dependent and ' $x$ ' is taken as the direction of movement of the device. In the present case, we only have an electrostatic force and the load force is zero. 


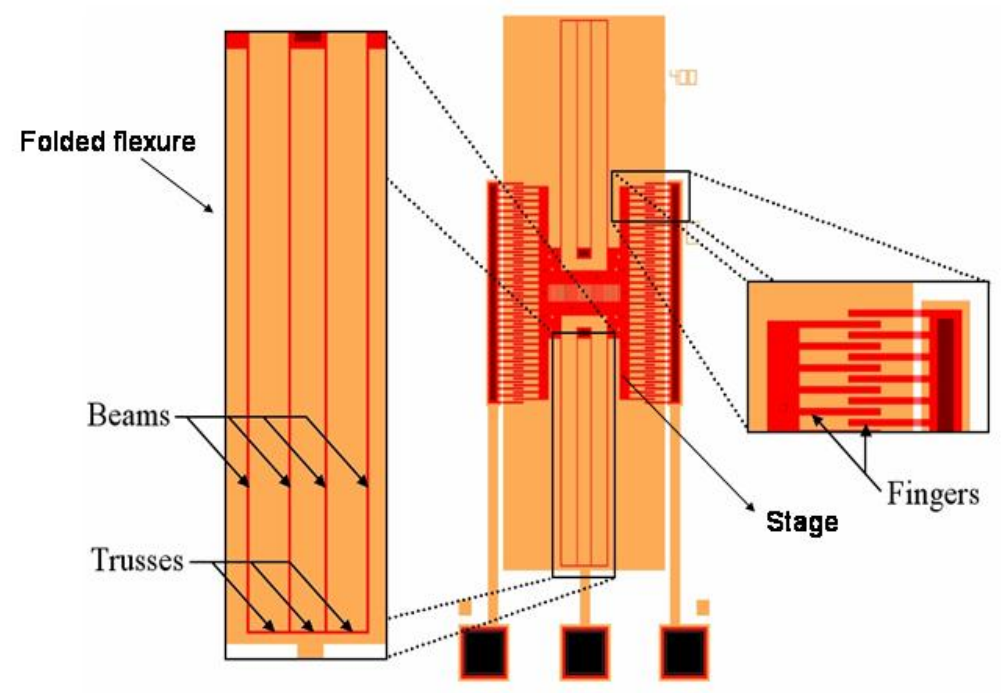

Figure 2.1: Features of LCR

Effective mass of the resonator is given by the equation 2.2 as given in [22]

$$
m=m_{s}+(1 / 4) m_{t}+(12 / 35) m_{b}
$$

where $m_{s}$ is the mass of the stage, and $m_{t}$ is the mass of truss, $m_{b}$ is the mass of the beam. The density of poly is $2330 \mathrm{~kg} / \mathrm{m}^{3}$ [8].

The beam and trusses which make the folded flexure, provide the restoring force for the suspended stage. The stiffness of the folded flexure is given by [14]

$$
k=\frac{2 E t w_{t}^{3}}{L_{b}^{3}} \frac{\bar{L}_{t}^{2}+14 \alpha \bar{L}_{t} L_{b}+36 \alpha^{2} L_{b}^{2}}{4 \bar{L}_{t}^{2}+41 \alpha \bar{L}_{t} L_{b}+36 \alpha^{2} L_{b}^{2}}
$$

For the current MEMS structure under consideration, $\mathrm{E}=$ Elasticity of the material $=160 \mathrm{E} 9$ [8, 39] for poly, $\bar{L}_{t}=L_{t} / L_{b}, L_{t}$ is the length of truss, $L_{b}$ is the length of beam, $w_{t}$ is the width of truss, $\mathrm{t}$ is the thickness of the substrate which is $2 \mu \mathrm{m}, w_{b}$ is the width of the beam equal to $2 \mu \mathrm{m}$, and $\alpha=w_{t}^{3} / w_{b}^{3}$

One of the most important parameters of a damped oscillator is the resonant frequency. This 
is given by the equation

$$
\omega=\sqrt{\frac{k}{m}}
$$

Therefore the resonant frequency in Hertz is given by

$$
f=\frac{1}{2 \Pi} \sqrt{\frac{k}{m}}
$$

The electrostatic force of attraction between the fixed stators and moving stage fingers is given by [37]

$$
F=-2.24 n \frac{\epsilon t}{g} V^{2}
$$

where $\mathrm{V}$ is the DC voltage applied to the stators, and also equal to the amplitude of the voltage applied to the stage which is of the form $\mathrm{V} \sin \omega t, \mathrm{n}$ is the number of fingers, and $\epsilon$ is the permittivity of free space which is equal to $8.856 \mathrm{e}-12$.

For the purposes of this work, it is important to determine the effects of the asymmetries in the flexure of the LCR. The asymmetries were expected to give the stage a nonideal motion which could lead to catastrophic faults. A model was developed previously [21], using lumped parameters. Fig.2.2 shows the simulink lumped parametric model used initially to characterize the devices. This could not be used to fully see and analyze the effects of the asymmetries in the flexure. Hence to have a better understanding of these effects, a solid model was required.

\subsection{Structural Mechanics}

The solid model was developed using finite element analysis. Finite element method has been used for the design [26, 25, 20, 13], stress analysis [18, 29, 35, 33], failure and fatigue [23, 40, 32] analysis etc. of various MEMS devices. Commercially available ANSYS software [17] was used to develop the model. Before we get into the details of the model, an overview of elasticity theory and finite element method is explained in the following subsections. 


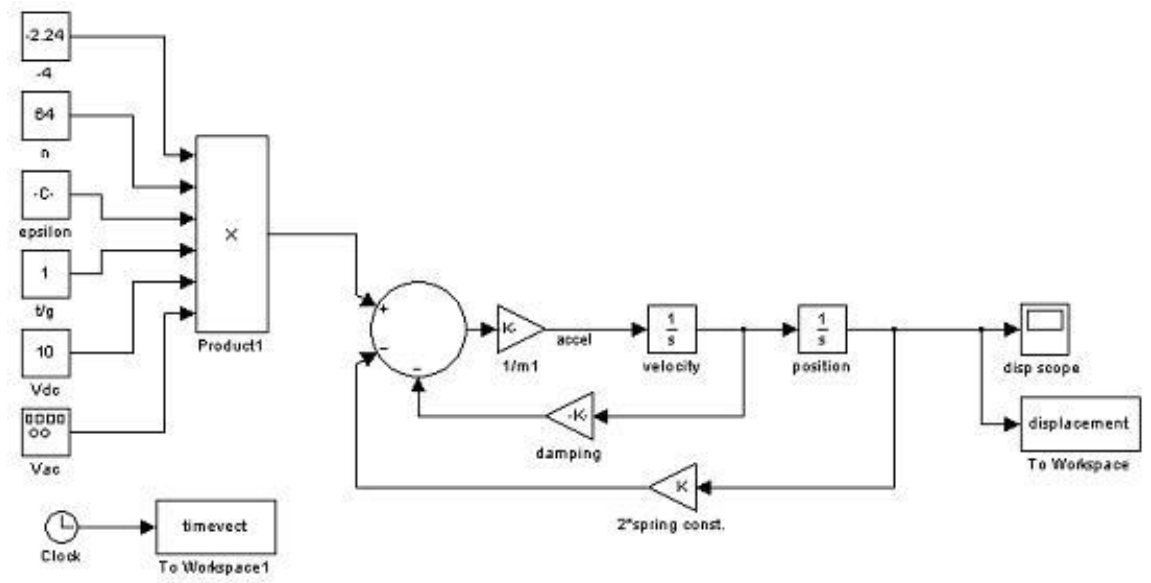

Figure 2.2: Lumped model of the LCR

\subsubsection{Elasticity Theory}

A mechanical structure's behavior is completely understood when its resonant modes, its displacement, stresses and strains are known. The structure's behavior is governed by Equilibrium, Kinematic and Constitutive equations [7]. Using these we determine displacements, stresses and strains of the system. The Fig.2.3 shows the body subjected to various stresses in the coordinate system shown.

The equilibrium equations are given by 2.7 [7], the index after the coma, indicates the rate of change of the stress in that direction.

$$
\begin{aligned}
& \sigma_{11,1}+\sigma_{21,2}+\sigma_{31,3}+f_{1}=0 \\
& \sigma_{12,1}+\sigma_{22,2}+\sigma_{32,3}+f_{2}=0 \\
& \sigma_{13,1}+\sigma_{23,2}+\sigma_{33,3}+f_{3}=0
\end{aligned}
$$

The kinematic equations which relate the displacements to strains are given by 2.8 [7]. Here $\mathrm{i}$ and $\mathrm{j}$ vary from 1 to 3 , giving a total of six equations for six strains.

$$
\epsilon_{i j}=1 / 2\left(u_{i, j}+u_{j, i}\right)
$$

The constitutive equations give the relation between strain and stress based on the type of material. Poly is a linearly isotropic material with Elastic modulus E $=160$ E9 and Poisson's 

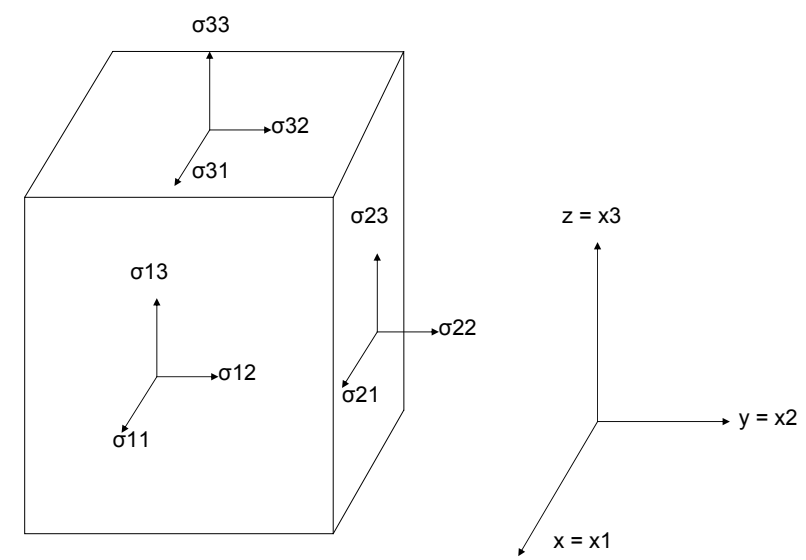

Figure 2.3: Stresses on a Solid Body

ratio $=0.22[8,39,34]$. The constitutive equations for a linearly isotropic element are given by the equations 2.9 [7].

$$
\left[\begin{array}{c}
\epsilon_{11} \\
\epsilon_{22} \\
\epsilon_{33} \\
\epsilon_{12} \\
\epsilon_{23} \\
\epsilon_{31}
\end{array}\right]=\left[\begin{array}{cccccc}
1 / E & -\nu / E & -\nu / E & 0 & 0 & 0 \\
-\nu / E & 1 / E & -\nu / E & 0 & 0 & 0 \\
-\nu / E & -\nu / E & 1 / E & 0 & 0 & 0 \\
0 & 0 & 0 & 1 / 2 G & 0 & 0 \\
0 & 0 & 0 & 0 & 1 / 2 G & 0 \\
0 & 0 & 0 & 0 & 0 & 1 / 2 G
\end{array}\right]\left[\begin{array}{c}
\sigma_{11} \\
\sigma_{22} \\
\sigma_{33} \\
\sigma_{12} \\
\sigma_{23} \\
\sigma_{31}
\end{array}\right]
$$

Solving a structure with the elasticity theory is often tedious and not very accurate. Finite element method was developed to overcome this problem.

\subsubsection{Finite Element Analysis}

Finite element analysis is the method of obtaining the solution of a body by dividing the body into small "elements". These elements are made of nodes which are interconnected to the 
nodes of other elements to form a mesh. Each element is solved independently, with boundary conditions and common node values taken from the neighboring elements. In the following paragraphs, finite element methods to solve various structural analysis is explained.

\subsubsection{Static Analysis}

Any static analysis on a body is governed by the stiffness of the body and the total load on it. The displacement of the body varies linearly with the force, and the general equation is given by $2.10[5]$.

$$
F=K x
$$

where $\mathrm{K}$ is the stiffness, $\mathrm{x}$ is the displacement and $\mathrm{F}$ is the total load acting on the body.

In finite element analysis the stiffness matrix determines the motion of the body. Hence the formulation of the global stiffness matrix is critical. The stiffness matrix depends on the type of element, number of nodes per element and the mesh that is used to solve the structure.

Every element is described by certain set of assumptions, depending on the dimensions and the general expected behavior. Since each element is solved only at the nodes, the behavior of the element anywhere else within the element is given by an interpolation function $\mathrm{H}$. $\mathrm{H}$ depends on the number of nodes in the element, as $\mathrm{H}$ is calculated as a function of values at the nodes [5].

The element strain in terms of the global displacements, is given by the equation 2.11

$$
\epsilon^{m}=[B]^{m} U
$$

where, $\epsilon$ is the element strain, $\mathrm{U}$ is the global displacement, $\mathrm{B}$ is determined using the kinematic relations given by 2.8 for every element. Using the principle that the internal stresses balance the external forces of all types, we can arrive at the equation 2.12. This equation is similar to the equation given by 2.10 . Therefore by comparing the two equations, the global stiffness matrix $\mathrm{K}$ is given by the equation $2.13[17,5]$.

$$
\begin{gathered}
{\left[\sum_{m} \int_{v^{m}}[B]^{T}[C][B] d V^{m}\right]\{U\}=\{R\}^{c}} \\
K=\left[\sum_{m} \int_{v^{m}}[B]^{T}[C][B] d V^{m}\right]
\end{gathered}
$$




\subsubsection{Modal Analysis}

This analysis is used for determining the resonant modes of the system. As mentioned earlier resonant frequency is one of the most important parameters of concern. The eigenvalues of a structure are solved by using the equation 2.14 [5]

$$
K \phi=\lambda M \phi
$$

where $\mathbf{M}$ is the mass matrix, $\phi$ and $\lambda$ are eigenvalues and eigenvectors respectively. The eigenvalues correspond to the squares of the resonant frequency modes [5] and the eigenvectors give the deformed shape of the structure when it is subjected to the corresponding resonant frequency(given by the eigenvalue).

\subsubsection{Harmonic Analysis}

The dynamic response of any structure is solved by the harmonic analysis. This analysis is done using the equation 2.1. In the commercially available ANSYS, $\beta$ can be given by describing the surrounding medium, or it can be approximated using the theory and giving it a fixed value. As described in the next chapter, the model that needs to be developed was not valid when the damping was ignored. The next section describes the evaluation of damping.

\subsubsection{Plane stress}

The element that is used in the model is the plane stress element. Plane stress assumes that the stress in one direction is negligible. This is usually used when one dimension of a body, is much smaller than the other two. The stress in the direction of the smallest dimension is ignored and a 3-D problem reduces to a 2-D problem. This makes the computation much faster. The kinematic and constitutive equations in 2.8 and 2.9 reduce to 2.15 and 2.16 respectively $[17,7]$.

$$
\begin{gathered}
\sigma_{33}=\tau_{23}=\tau_{13}=0 \\
{\left[\begin{array}{c}
\epsilon_{11} \\
\epsilon_{22} \\
\epsilon_{12}
\end{array}\right]=\left[\begin{array}{cc}
\frac{\partial}{\partial x} & 0 \\
0 & \frac{\partial}{\partial y} \\
\frac{\partial}{\partial y} & \frac{\partial}{\partial x}
\end{array}\right]\left[\begin{array}{l}
u \\
v
\end{array}\right]}
\end{gathered}
$$




$$
\left[\begin{array}{c}
\epsilon_{11} \\
\epsilon_{22} \\
\epsilon_{12}
\end{array}\right]=\left[\begin{array}{ccc}
1 / E & -\nu / E & 0 \\
-\nu / E & 1 / E & 0 \\
0 & 0 & 2(\nu+1) / E
\end{array}\right]\left[\begin{array}{c}
\sigma_{11} \\
\sigma_{22} \\
\sigma_{12}
\end{array}\right]
$$

\subsection{Damping}

Damping $\beta$ used in equation 2.1 is replaced by $\mathrm{C}$ in the rest of the section, and $\beta$ is used for stiffness damping multiplier. Also $\mathrm{u}$ is used instead of $\mathrm{x}$ to represent the direction of motion. To avoid any confusion the equation 2.1 is rewritten with the current notation in equation 2.17.

$$
\left\{F^{a}\right\}=[M]\{\ddot{u}\}+[C]\{\dot{u}\}+[K]\{u\}
$$

From [17], we get the equation for damping as 2.18

$$
[C]=\alpha[M]+\left(\beta+\beta_{c}\right)[K]+\sum_{j=1}^{N M A T} \beta_{j}\left[K_{j}\right]+\sum_{N=1}^{N E L}\left[C_{k}\right]+\left[C_{\xi}\right]
$$

where $[\mathrm{C}]$ is the structure damping matrix, $\alpha$ is the constant mass matrix multiplier, $[\mathrm{M}]$ is the structure mass matrix, $\beta$ is the constant stiffness matrix multiplier, $\beta_{c}$ is the variable stiffness matrix multiplier, $[\mathrm{K}]$ is the structure stiffness matrix, NMAT is the number of materials with DAMP input, $\beta_{j}$ is the constant stiffness matrix multiplier for material $\mathrm{j}, K_{j}$ is the portion of structure stiffness matrix based on material $\mathrm{j}$, NEL is the number of elements with specified damping, $C_{k}$ is the element damping matrix, $C_{\xi}$ is the frequency-dependent damping matrix.

The material under consideration here doesn't have any built-in material damping. Therefore the term $\sum_{j=1}^{N M A T} \beta_{j}\left[K_{j}\right]$ in the equation 2.18 may be eliminated. The damping for every element is uniform, due to uniform air density around the structure; therefore any extra damping need not be specified for a particular element. Hence the term $\sum_{N=1}^{N E L}\left[C_{k}\right]$ can be ignored. From [1] it is clear that the value of $\alpha$ is very small and the mass matrix multiplier term can also be dropped. Therefore the equation 2.18 simplifies to equation given by 2.19

$$
[C]=\left(\beta+\beta_{c}\right)[K]+\left[C_{\xi}\right]
$$


Combining the variable and constant stiffness matrix gives a single parameter, variable matrix multiplier, $\beta_{c}$. This is given in [17] as equation 2.20.

$$
\beta_{c}=\frac{\zeta}{\pi f}
$$

where $\zeta$ is the Damping ratio, $\mathrm{f}$ is the frequency in between the beginning and ending frequency entered in the harmonic analysis.

$C_{\xi}$ is a function of $\xi_{d}$ given in [17] by 2.21

$$
\left\{U_{i}\right\}^{T}\left[C_{\xi}\right]\left\{U_{i}\right\}=4 \pi f_{i} \zeta_{i}^{d}
$$

where $\left\{U_{i}\right\}$ is the shape of mode $\mathrm{i}, \zeta_{i}^{d}=\zeta+\zeta_{m i}$. The term $\zeta_{m i}$ is the modal damping ratio, a material dependent property. This is ignored as there is no material dependent damping for polysilicon and hence $\zeta_{m i}=0$.

Hence it is clear that the damping value can be calculated by giving $\zeta$ as the input. $\zeta$ is given by the equation 2.22; where $C_{c}$ is the critical damping. Critical damping is given by the equation 2.23. Here $\mathrm{k}$ and $\mathrm{m}$ are the lumped parameters given by 2.3 and 2.2 respectively. Therefore by calculating $\mathrm{C}$, the value of the damping ratio $\zeta$ can be computed and given as an input in ANSYS.

$$
\begin{gathered}
\zeta=\frac{C}{C_{c}} \\
C_{c}=2 \sqrt{\mathrm{km}}
\end{gathered}
$$

Therefore

$$
\zeta=C /(2 \sqrt{k m})
$$

To calculate this value, the equation for the quality factor as given in [27], derived for the comb resonator given by 2.25 is used

$$
Q=\sqrt{(k m) / C}
$$

From 2.24 and 2.25 it can be deduced that

$$
\zeta=\frac{1}{2 Q}
$$


The quality factor of the resonator can be calculated by using the equation in [42],

$$
Q=\frac{\mu}{\sqrt{(k m)}}\left[\frac{A_{c}}{d_{c}}+\frac{\alpha A q}{d\left(1+\frac{d}{\delta}\right)}\right]
$$

where $\mu$ is the viscosity of air $=1.88 \mathrm{e}-5, A_{c}$ is the Area of comb overlap, $d_{c}$ is the comb gap $A_{q}=A_{s}+0.5\left(A_{t}+A_{b}\right), A_{s}$ is the Area of Stage, $A_{t}$ is the Area of Truss, $A_{b}$ is the Area of beam, $\mathrm{d}$ is the distance of the structure above the substrate, $\delta$ is the penetration depth given by $\delta(\omega)=\sqrt{(} 2 \nu / \omega)$, where $\omega$ is the resonant frequency, $\nu$ is the kinetic viscosity of air given by $\nu=\mu / \rho, \rho$ is the density of air $=1.22, \alpha$ is the non-ideality factor, which depends on the plate geometry, that can be determined using experimental values as shown in the next chapter. 


\section{Chapter 3}

\section{MEMS sensitivity analysis and validation}

This chapter deals with the key simulation results and fault predictions. Section 3.1 deals with the development of the solid model, various elements and the type of meshing used. Section 3.2 describes the validation of the model with the already known theory. Section 3.3 talks about the fabrication tolerances and the variations caused due to the same. Section 3.4 deals about utilizing the current solid model for fault detection.

\subsection{Development of solid model}

Polysilicon was modeled as a linearly isotropic material as mentioned in [34]. The modulus of elasticity of the material is taken to be $160 \mathrm{E} 9$ and poisson's ratio to be 0.22 .

The precision of a model depends on the choice of the element and the mesh used. In all the models explored, a smart mesh tool is used. This detects the edges of a surface and automatically refines the mesh at the boundaries. The model was meshed using various elements and as a preliminary validation, static displacement and resonant frequency values predicted from the theory were used. The expected values are $2.25 \mu \mathrm{m}$ and $2591 \mathrm{~Hz}$, for a $450 \mu \mathrm{m}$ flexure length device.

A two noded beam element was used to mesh the beams and an eight noded plane stress element was used to mesh the rest of the model. Plane stress elements can be given a thickness 


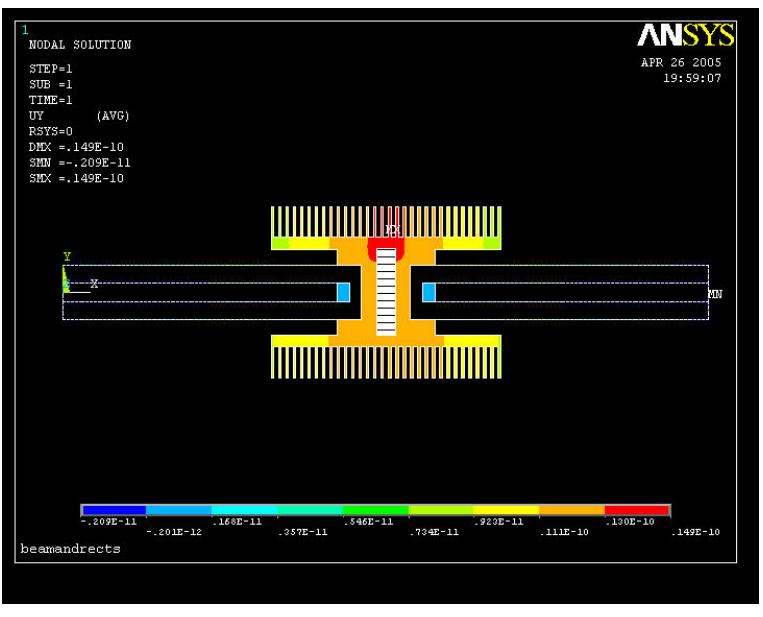

(a) Beam and Plane stress elements

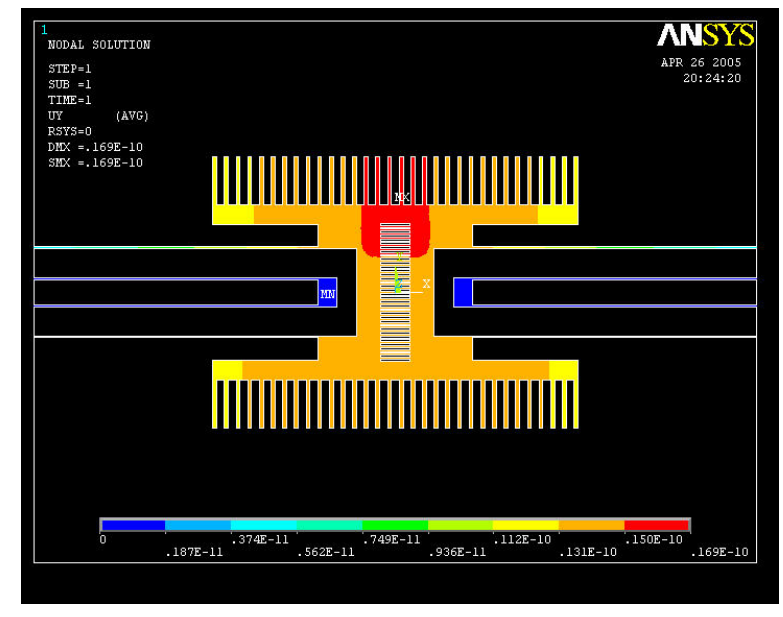

(b) Plane strain and Plane stress elements

Figure 3.1: Contours

with the current model. A thickness of $4 \mu \mathrm{m}$ was used for meshing the anchors, as they are $4 \mu \mathrm{m}$ deeper than the top surface of poly 1 . As everything else is suspended and the thickness of poly 1 is $2 \mu m$, they were modeled with plane stress elements with a thickness of $2 \mu m$ with this model. The static displacement and resonant frequency with this model, were predicted to be $0.149 \mathrm{e}-$ $10 \mathrm{~m}$ and $576 \mathrm{kHz}$ respectively. Fig.3.1(a), shows the contour plot of the static displacement. The beam element is a two noded element, and is not very accurate when coupled with higher noded elements. Therefore this model could not accurately predict the parameters.

Since the length of the beam is much larger when compared with the other two dimensions, it can be approximated to have a plane strain condition. Hence a plane strain element was used to mesh the beams. The static displacement was found to be $1.69 \mathrm{e}-10 \mathrm{~m}$ and the resonant frequency was $8395.5 \mathrm{~Hz}$ with this model. The thickness for the plane strain element cannot be defined in ANSYS and in the absence of an explicit value for the thickness, it assumes a value of $1 \mathrm{~m}$ (whereas the actual thickness is only $2 \mu \mathrm{m}$ ) and hence the model was not good to predict the behavior. Fig.3.1(b) shows the displacement contour when the plane strain elements are used.

The entire model was then meshed using plane stress elements. The model could then predict the resonant frequency, but the static displacement $(0.447 \mathrm{e}-11 \mathrm{~m})$ was not accurate. Later, a 


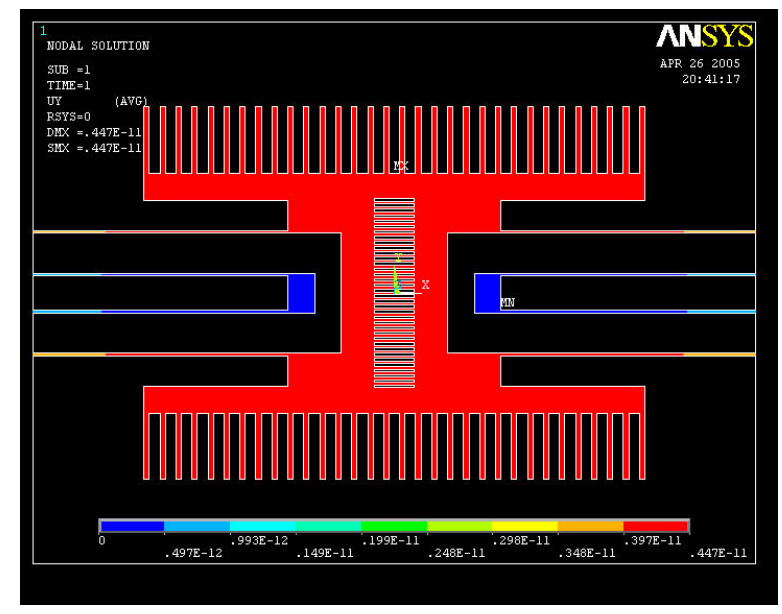

(a) Plane stress elements with uniform thickness

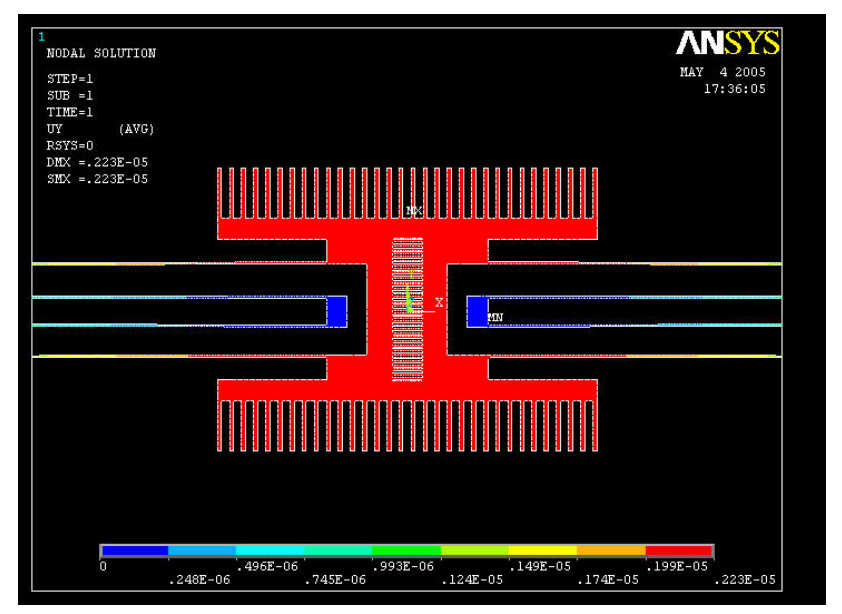

(b) Plane stress elements with $4 \mu m$ thickness for anchors

Figure 3.2: Contours

thickness of $4 \mu \mathrm{m}$ was used to mesh the anchors and $2 \mu \mathrm{m}$ was used for the rest of the model. It was found to match both the resonant frequency and static displacement. The Fig.3.2 shows the contours of the two cases. The table 3.1 summarizes the various models and elements used and the model that gives accurate results.

Fig.3.3 shows the meshed final solid model. The model takes about 30 seconds to run a static analysis and about 2 minutes to run a modal analysis when used to extract 5 modes of vibration. 


\begin{tabular}{|c|c|c|}
\hline Type of device & Static displacement & Resonant Frequency \\
\hline $\begin{array}{c}\text { Beam and plane stress elements, } \\
\text { with anchor thickness } 4 \mu m\end{array}$ & $0.149 \mathrm{e}-10$ & $576 \mathrm{kHz}$ \\
\hline $\begin{array}{c}\text { Beams with plane strain, } \\
\text { anchor with } 4 \mu m \text { plane stress element }\end{array}$ & $1.69 \mathrm{e}-10$ & 8395.5 \\
\hline $\begin{array}{c}\text { All the areas with plane stress element } \\
\text { with no thickness specified }\end{array}$ & $.447 \mathrm{e}-11$ & $2591 \mathrm{~Hz}$ \\
\hline $\begin{array}{c}\text { All areas with plane stress element of } 2 \mu m \text { and } \\
\text { anchor with } 4 \mu m \text { thickness }\end{array}$ & $2.25 \mu m$ & $2591 \mathrm{~Hz}$ \\
\hline
\end{tabular}

Table 3.1: Summary of elements used

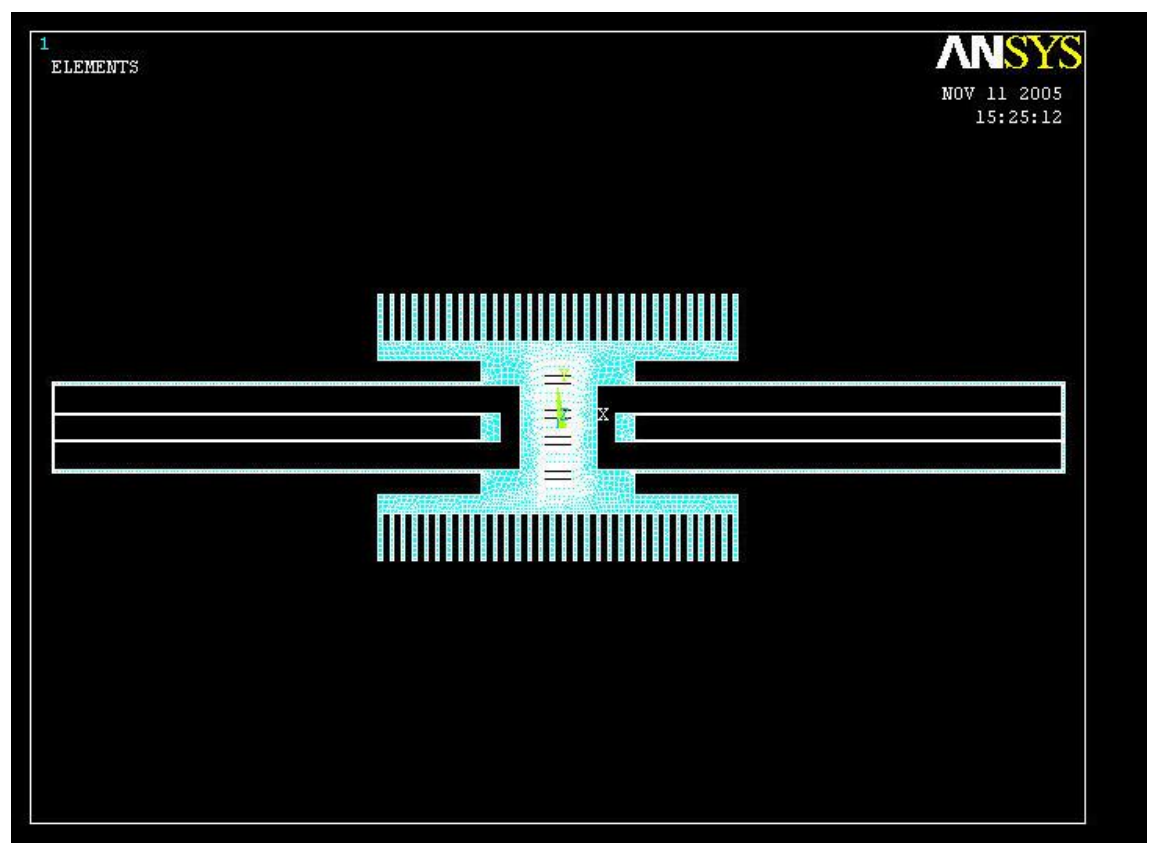

Figure 3.3: Meshed Model 


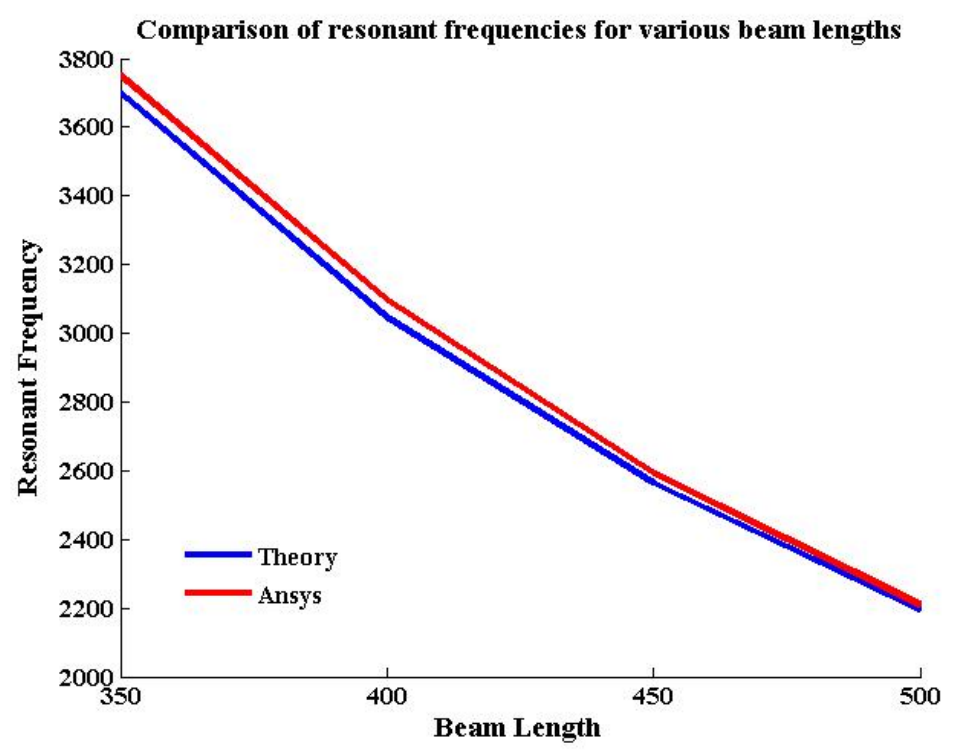

Figure 3.4: Theory and Ansys comparison

\subsubsection{Initial validation}

The resonant frequencies at various beam lengths were calculated using equations 2.2, 2.3, 2.5 and these were compared with the resonant frequencies obtained from the model. The table 3.2 shows the values of the resonant frequency. The Fig.3.4 shows the comparison between the two and it can be observed that the values predicted from the model closely match those predicted by the theory.

\begin{tabular}{|c|c|c|c|}
\hline $\begin{array}{c}\text { Length of the Beam } \\
\text { in } \mu \mathrm{m}\end{array}$ & $\begin{array}{c}\text { Expected resonant } \\
\text { frequency }(\mathrm{Hz})\end{array}$ & $\begin{array}{c}\text { Resonant frequency } \\
\text { from the model }(\mathrm{Hz})\end{array}$ & Percentage error \\
\hline 350 & 3698 & 3754 & 1.5 \\
\hline 400 & 3048 & 3095 & 1.5 \\
\hline 450 & 2592 & 2591 & 0.2 \\
\hline 500 & 2194 & 2210 & 0.73 \\
\hline
\end{tabular}

Table 3.2: Theory and Ansys comparison 


\subsubsection{Residual Stress}

Every MUMPS run has a residual stress in each of the layers which occur due to fabrication processes. According to the run-data posted on the MEMSCap portal, the average residual stress is about $7 \mathrm{MPa}$ (Compression) for the poly1 layer. This was given as a initial stress condition for the model and the model was re-run. It was found that the neither the displacement nor the resonant frequency varied more than $0.44 \%$, which is negligible. Hence in further analysis, residual stress is ignored. Fig.3.5(a) and Fig.3.5(b), show the displacement contour plots, when residual stress is ignored and considered respectively.

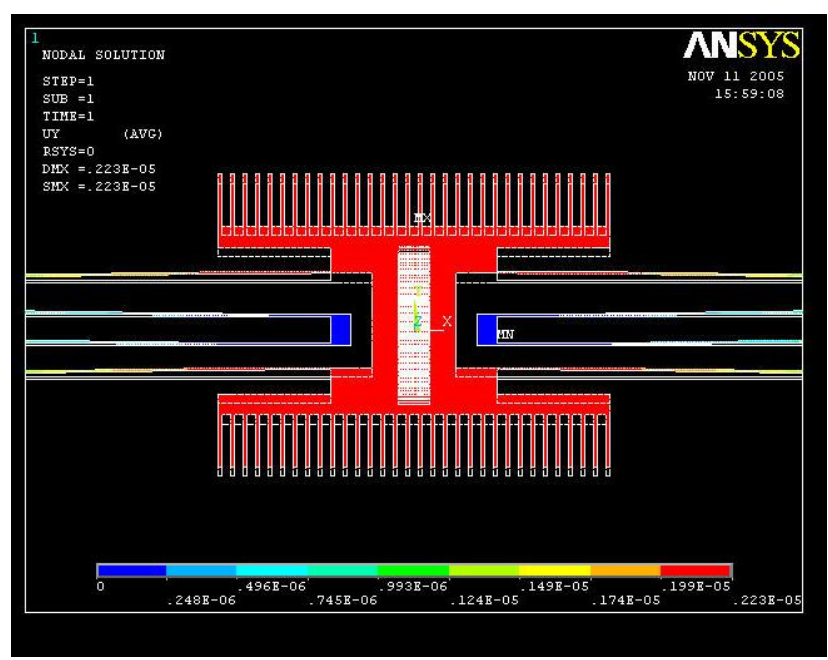

(a)Displacement contour when residual stress is ignored

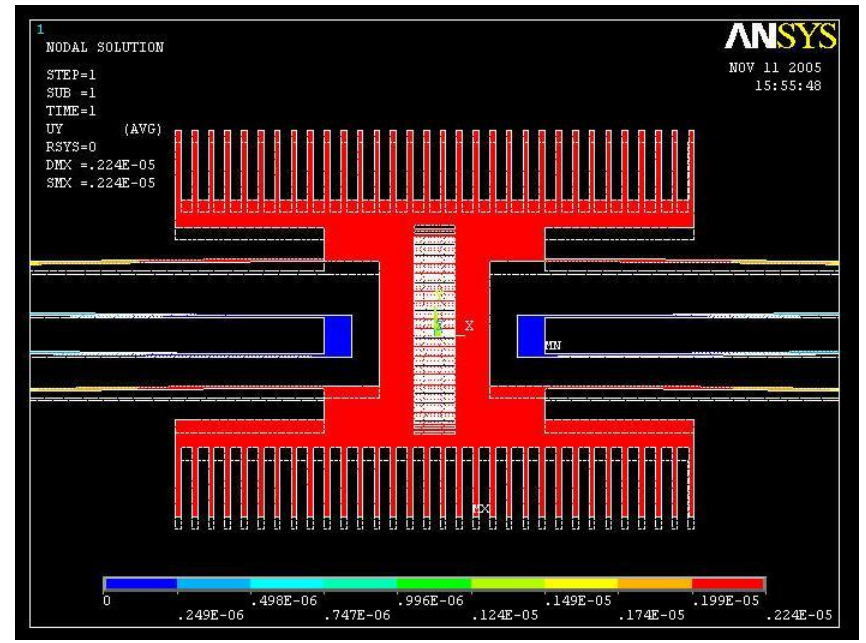

(b) Displacement when residual stress is considered

Figure 3.5: Residual Stress: Displacement Plots

\subsection{Validation}

After the initial validation was done, a further validation was required to authenticate the model. Experimentally determined frequency response of a $450 \mu \mathrm{m}$ flexure length device was used to evaluate the results obtained from ANSYS simulation.

The solid model was run through various frequencies and the experimental data was 


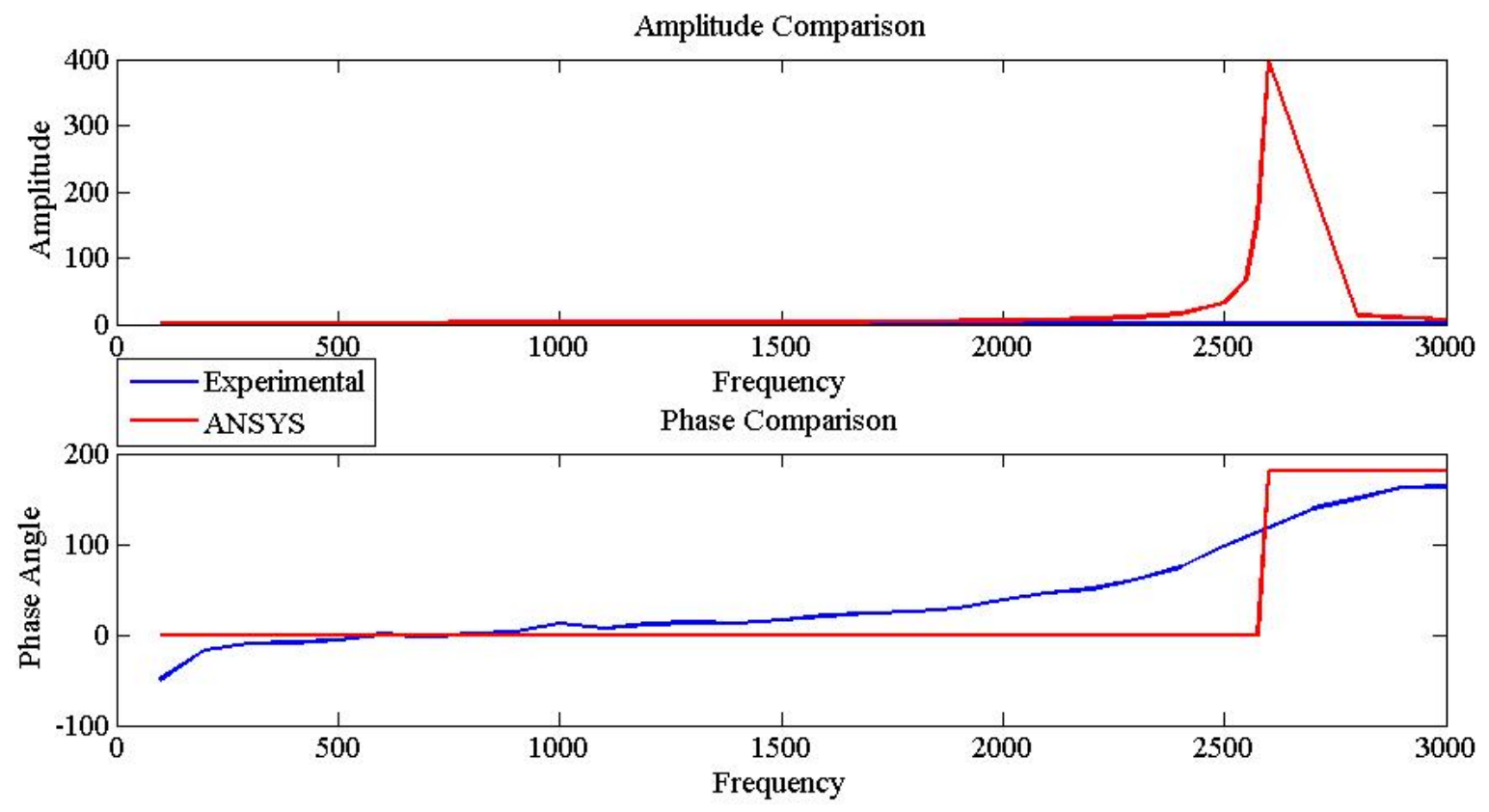

Figure 3.6: Frequency response when damping was ignored

compared. Fig.3.6, shows the response obtained when the damping was ignored. As can be observed from this figure, damping needs to be taken into consideration.

Therefore damping was calculated using the equations 2.22, 2.25 and 2.27. As mentioned earlier the non-ideality factor, $\alpha$ in the equation 2.27 depends on the geometry of the stage. The damping ratio obtained from the experimental data was taken to estimate the non-ideality factor. The non-ideality factor was found to be 1.67 for this geometry. This value was used to further calculate the damping factors of other devices and these were compared with the values obtained from experiments. The damping factors were found to be in good agreement with the calculated values. With the calculation of the correct damping value based on the geometry of the device, the frequency response of the model was simulated again and compared with the experimental data. Fig.3.7 and Table 3.3 show the comparison between ANSYS and the experimental data. As can be observed from the Fig.3.7 the experimental data and the Ansys data match very closely. 


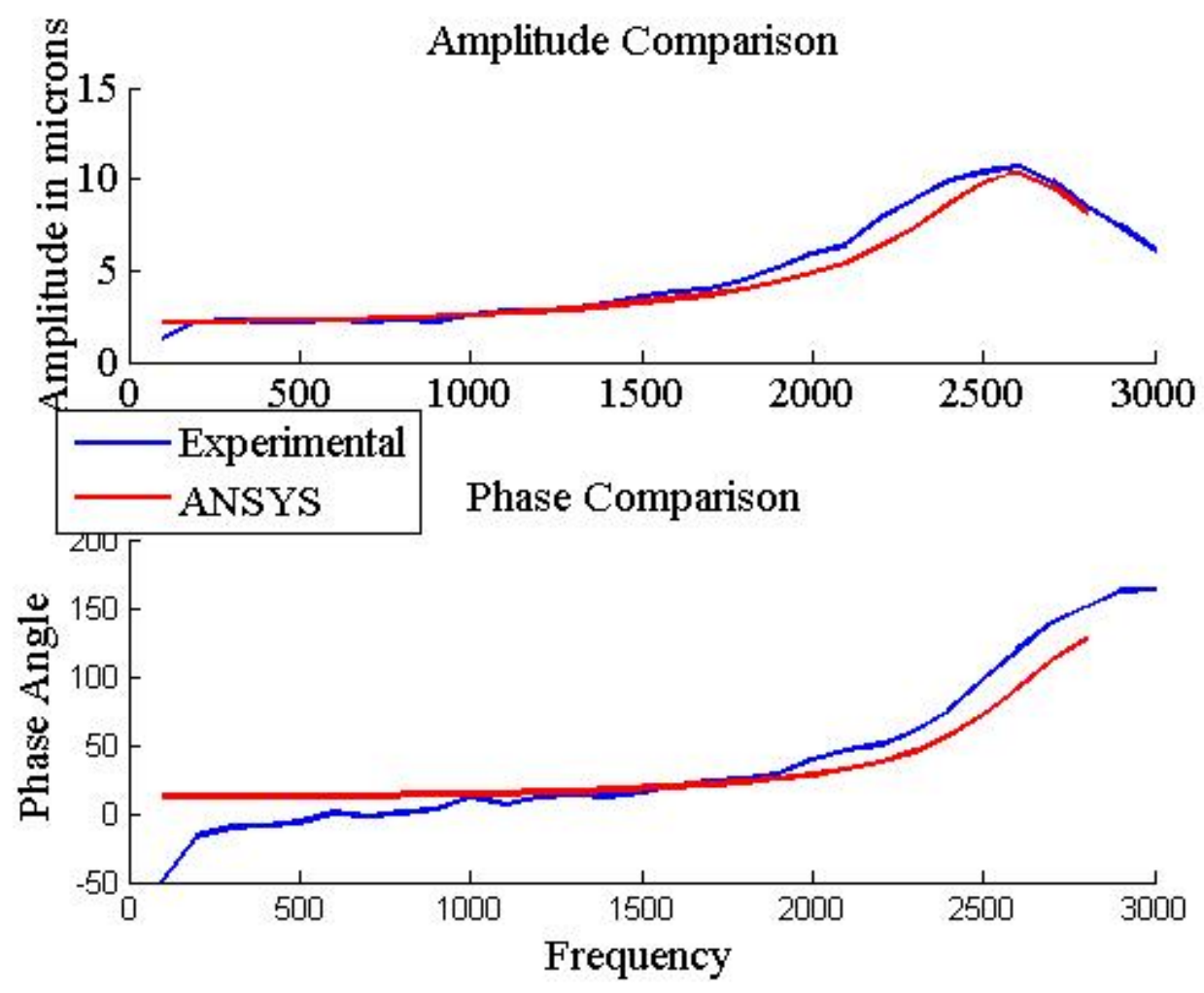

Figure 3.7: ANSYS and Experimental comparison

\subsection{Fabrication Tolerance}

A Fault or defect is something that effects any device's normal operation or any parameter of the device that may be of interest, depending on the application. Detection of these defects increases the reliability of the device. As mentioned earlier, our device designs are fabricated at the MEMSCap Foundry, and at present the best tolerance acheivable from this process is $\pm 0.3 \mu \mathrm{m}$. This gives wide variations in parameters such as maximum displacement, resonant frequency etc. Any device with other defects might also have its parameters within this range. This is called behavior-masking [13], which makes the detection of these defects, a challenge. Fig.3.8 shows the experimental frequency response, and it also gives the maximum change in the frequency response due to the fabrication tolerance. Table 3.4 gives the range of resonant frequency for different flexure lengths. 


\begin{tabular}{|c|c|c|c|c|}
\hline $\begin{array}{c}\text { Frequency } \\
\mathrm{Hz}\end{array}$ & $\begin{array}{c}\text { Experimental } \\
\text { Amplitude }(\mu \mathrm{m})\end{array}$ & $\begin{array}{c}\text { Ansys } \\
\text { Amplitude }(\mu \mathrm{m})\end{array}$ & $\begin{array}{c}\text { Experimental } \\
\text { Phase }\end{array}$ & $\begin{array}{c}\text { Ansys } \\
\text { Phase }\end{array}$ \\
\hline 1700 & 3.96 & 3.66 & 18.12 & 20.89 \\
1800 & 4.46 & 3.96 & 23.37 & 22.69 \\
1900 & 5.18 & 4.36 & 25.09 & 25.07 \\
2000 & 5.91 & 4.8 & 29.10 & 28.14 \\
2100 & 6.38 & 5.49 & 38.84 & 32.14 \\
2200 & 7.86 & 6.30 & 46.29 & 37.72 \\
2300 & 8.86 & 7.34 & 50.30 & 48.47 \\
2400 & 9.95 & 8.59 & 60.61 & 56.54 \\
2500 & 10.4 & 10.30 & 74.91 & 72.02 \\
2600 & 10.68 & 10.40 & 97.86 & 91.47 \\
2700 & 9.87 & 9.6 & 119.73 & 111.28 \\
2800 & 8.47 & 8.17 & 139.34 & 127.49 \\
\hline
\end{tabular}

Table 3.3: ANSYS and Experimental data comparison

\begin{tabular}{|c|c|c|c|}
\hline Length of the flexure & Minimum & Standard & Maximum \\
\hline 350 & 2875 & 3750 & 4764 \\
400 & 2353 & 3095 & 3898 \\
450 & 1970 & 2591 & 3260 \\
500 & 1886 & 2210 & 2677 \\
\hline
\end{tabular}

Table 3.4: Range of resonant frequency within the fabrication tolerances

\subsubsection{Sensitivity analysis}

Any fault or defect can only be determined and analyzed when the parameter, whose slight variation causes huge changes in any measurement of interest, can be found. In the present case the measurements of interest are resonant frequency, static displacement and frequency response. 

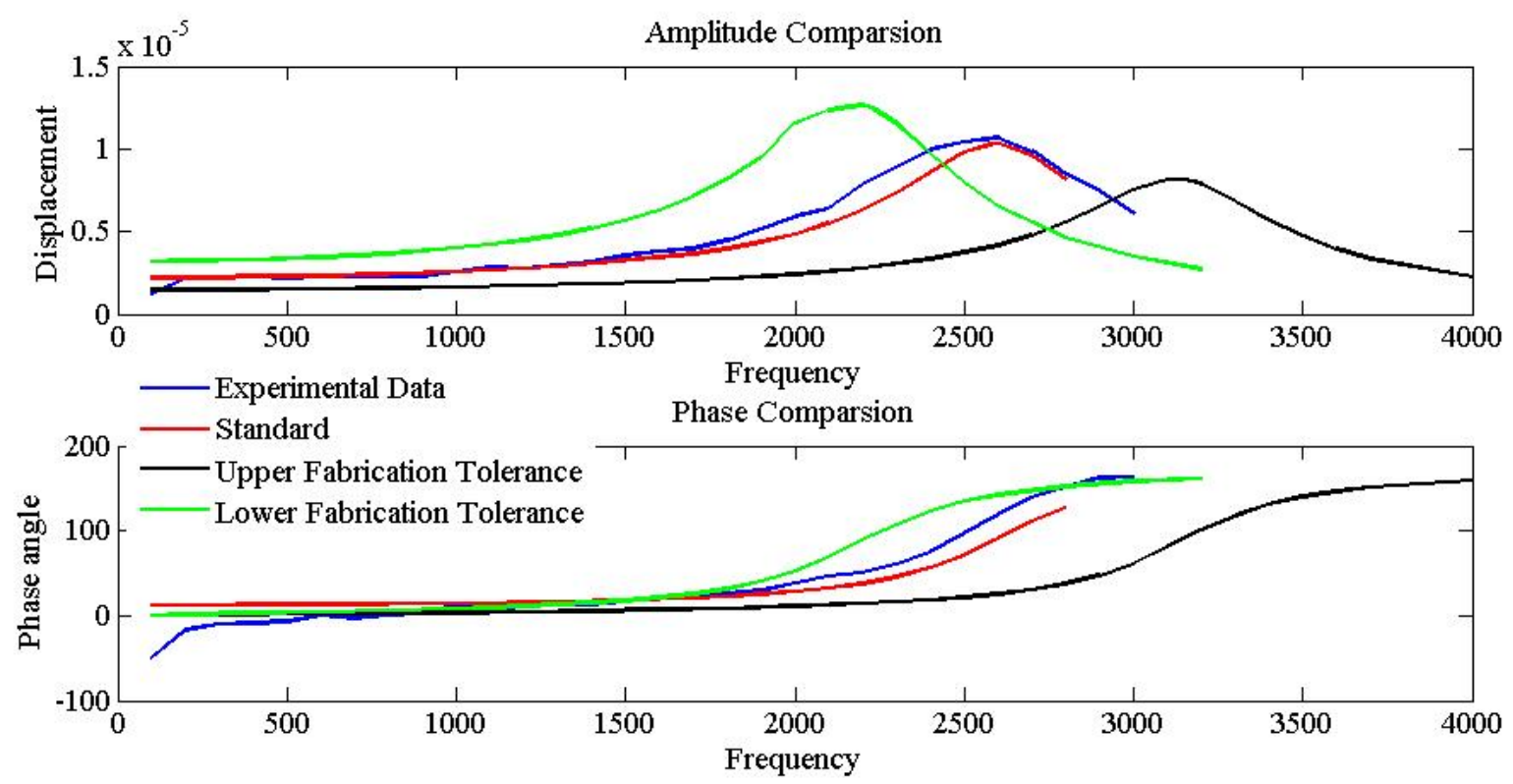

Figure 3.8: Fabrication Tolerance

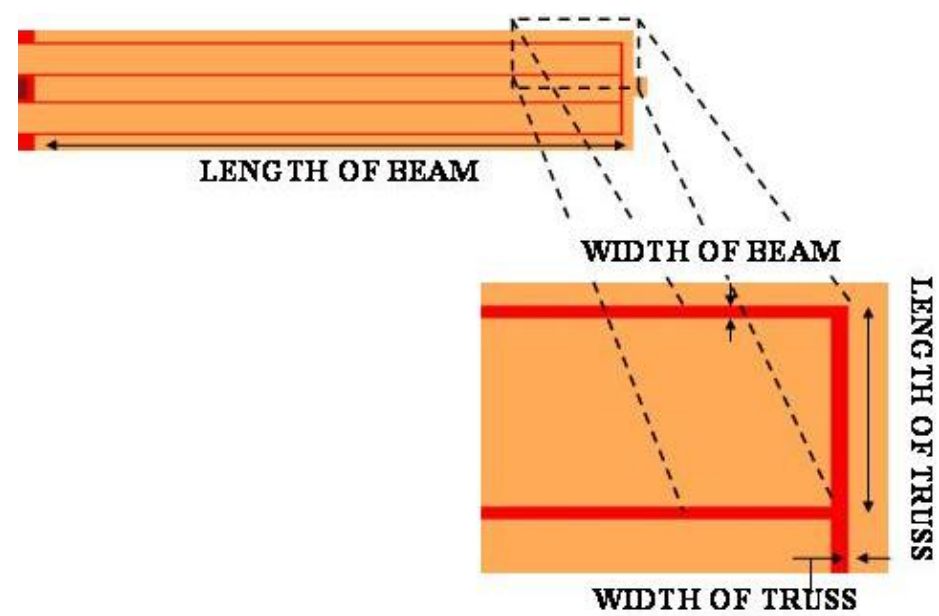

Figure 3.9: Parameters analyzed

The validation of the model was hence done and this was used to determine the most sensitive parameter. The parameters under consideration are length and width of the beam as well as length 


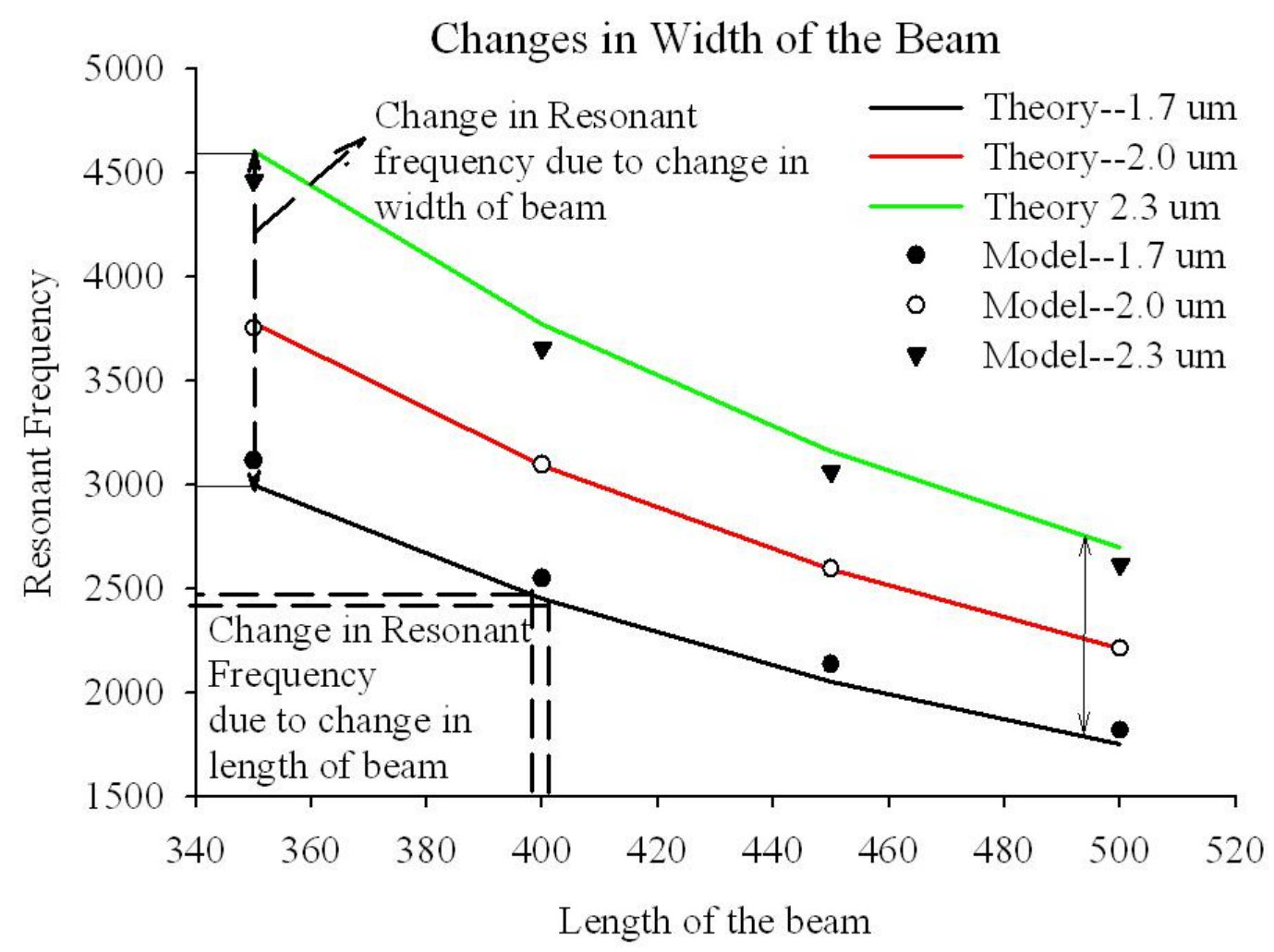

Figure 3.10: Variation in resonant frequency with Length of beam and Width of the beam

and width of the truss as shown in Fig.3.9. Resonant frequency was taken as the measure to determine the parameter sensitivity. The sensitivity analysis was done using both the theory and ANSYS model, as additional verification for the model. From the Fig.3.10 it can be observed that about $15 \%$ (caused due to the fabrication tolerance) change in width of the beam causes about an equal percentage change in resonant frequency, where as the change in resonant frequency due the changes in the length of the beam is negligible. From the Fig.3.11 it can be observed that for $15 \%$ change in the width of the truss, the change in resonant frequency is about $1.4 \%$. From these observations, it can be concluded that the width of the beam is the most sensitive parameter. Table 3.5 summarizes the effect of each of the parameter considered. 


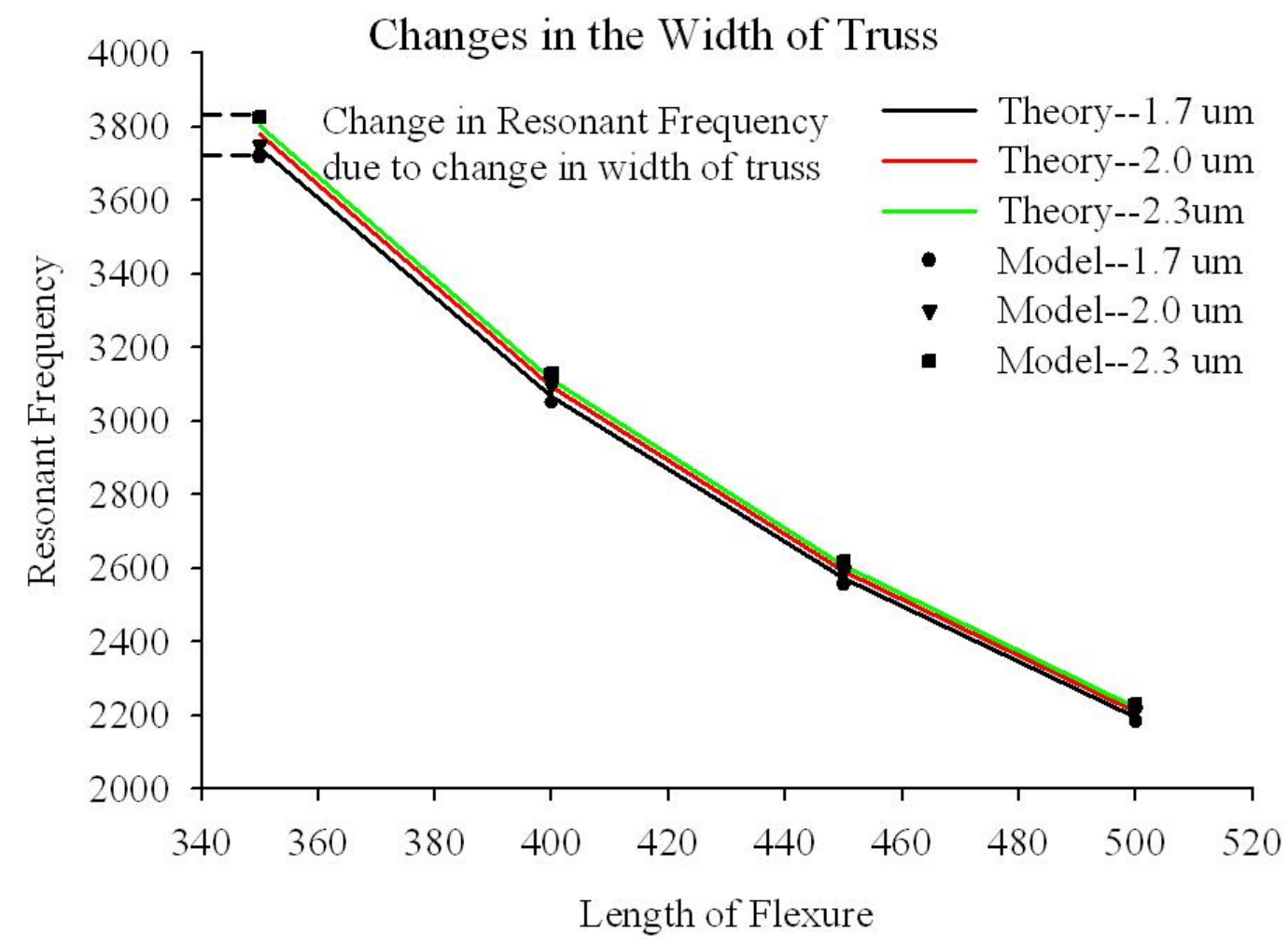

Figure 3.11: Variation in resonant frequency with Length of beam and Width of the truss

\begin{tabular}{|c|c|}
\hline Parameter & $\begin{array}{c}\text { \% Change in resonant frequency } \\
\text { when parameter is changed } \\
\text { within the fabrication error }\end{array}$ \\
\hline Width of truss & 2.87 \\
Length of truss & -0.03 \\
Width of beam & 37.76 \\
Length of beam & -0.2 \\
\hline
\end{tabular}

Table 3.5: Percentage change in resonant frequency 


\subsection{MEMS fault prediction}

As the most sensitive parameter was determined, the effects of asymmetry due to changes in the width of the beam were examined. The beams of the flexure were classified into two types. The one connected to the anchor is referred to as the anchor beam, and the other beam which is connected to the stage as corner beam. Fig.3.12 shows the classification of the beams. In this

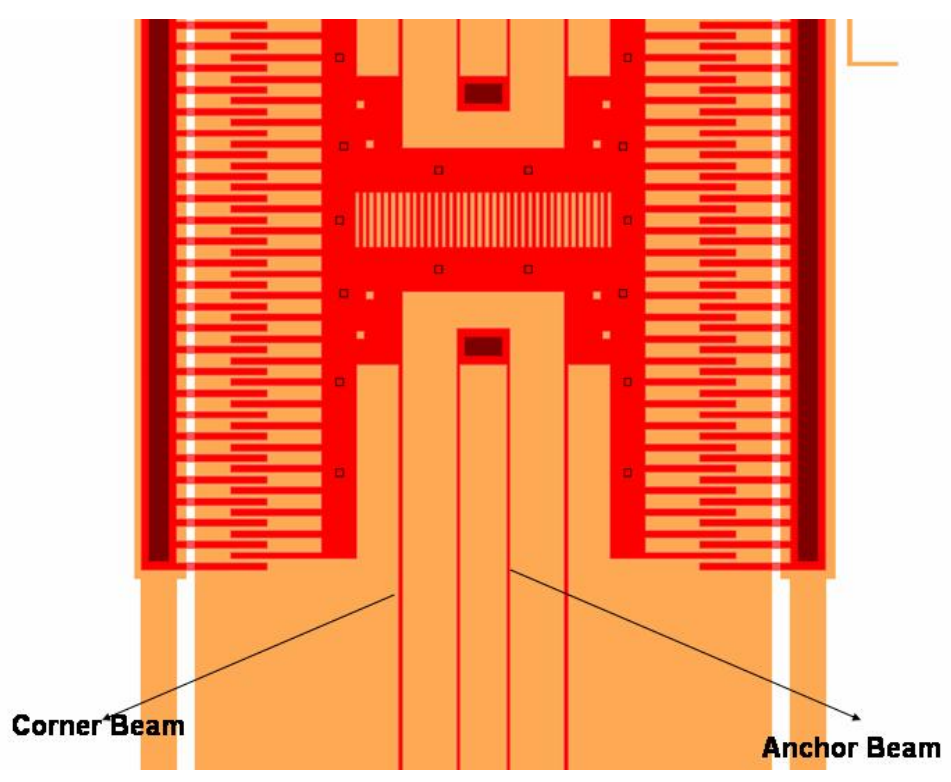

Figure 3.12: Beam Classification

section we will see how two classes of defects effect device performance and how they can be detected. These are

1.) Defects due to process etch variations and

2.) Defects due to point adhesion of flexures arising from particulate or stiction.

To simulate defects due to etch variation, the width of one of the beams was varied, with all the other widths kept constant, and the variation in resonant frequency and peak amplitude [Peak Amplitude is the maximum displacement of the device, with its current mass, spring constant and damping], were analyzed. It was observed that both resonant frequency and peak amplitude of the defective asymmetric devices lie within the resonance frequency envelope of the defect free devices arising from fabrication tolerances. Even a gross change up to $50 \%$ of the beam width 


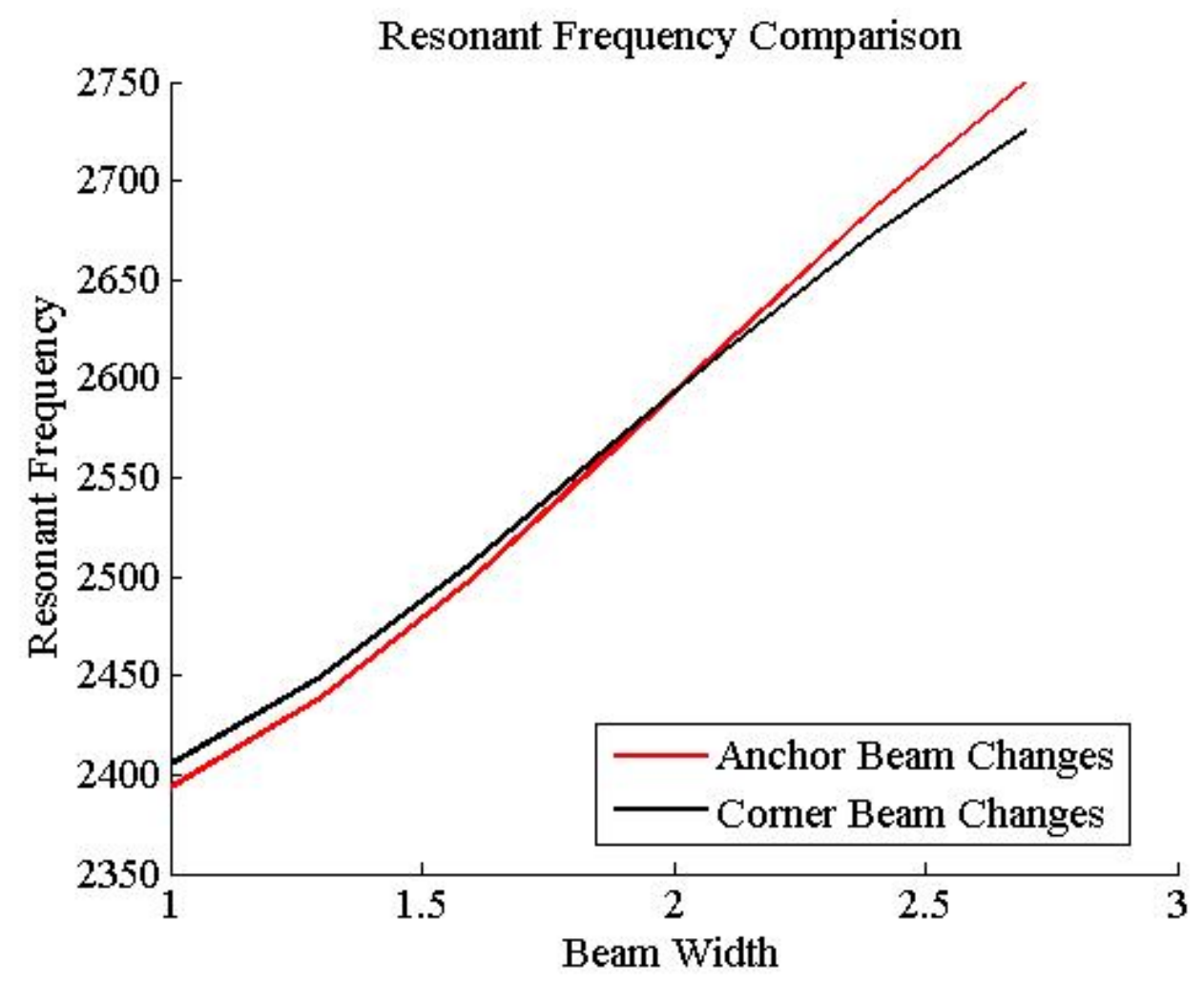

Figure 3.13: Resonant frequency variation with the change in width of a single beam

\begin{tabular}{|c|c|c|}
\hline Width of Beam & $\begin{array}{c}\text { Anchor beam } \\
\text { Resonant frequency }\end{array}$ & $\begin{array}{c}\text { Corner Beam } \\
\text { Resonant Frequency }\end{array}$ \\
\hline 1 & 2393.1 & 2404.7 \\
1.3 & 2438.6 & 2449.3 \\
1.6 & 2499.1 & 2507.1 \\
1.9 & 2568.6 & 2571.3 \\
2.1 & 2616.7 & 2613.9 \\
2.4 & 2686.4 & 2673.5 \\
2.7 & 2749.8 & 2725.5 \\
\hline
\end{tabular}

Table 3.6: Resonant frequency variation with change of single beam width 


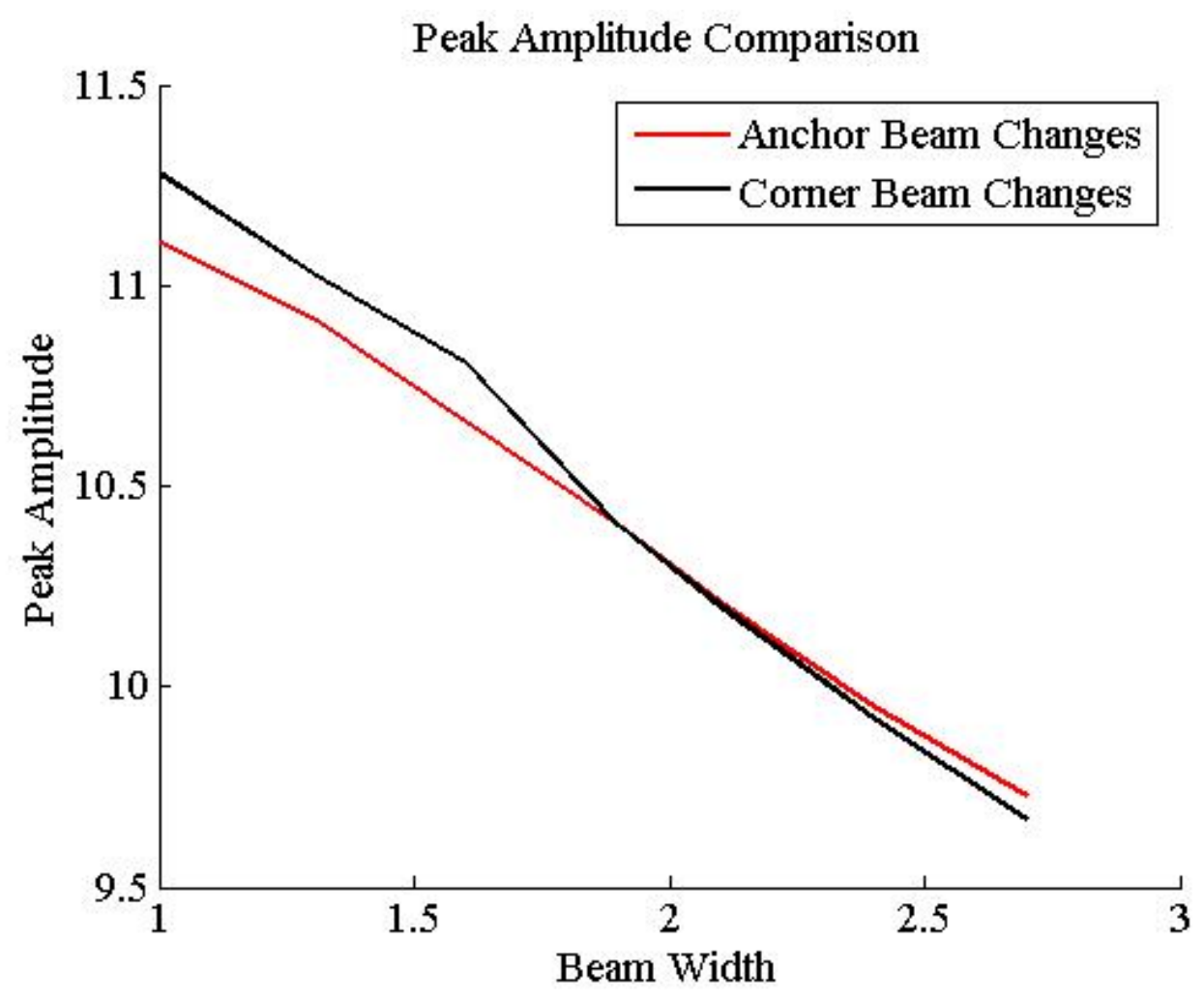

Figure 3.14: Peak amplitude variation with the change in width of a single beam

could not be detected by analyzing these parameters. Fig.3.13 and Fig.3.14 illustrate the fact that resonant frequency and peak amplitude are not good measures for the detection of asymmetries. Table 3.6 and 3.7, also illustrate the same fact.

Point adhesion of the flexure structure can be modeled as an extra anchor to the device at that point. The range of static displacement and resonant frequency due to the fabrication error is $1.47 \mu \mathrm{m}$ to $3.2 \mu \mathrm{m}$ and $1970 \mathrm{~Hz}$ to $3560 \mathrm{~Hz}$ respectively for a $450 \mu \mathrm{m}$ beam length device. Any kind of variations outside this range are easily detectable, but when a faulty device's parameters lie within this range neither resonant frequency nor static displacement can be used for the detection. Table 3.8, shows the values of resonant frequency and static displacement when the particle is located under: Stage Comb and Beams

As can be observed point adhesion under the beam cannot be detected with a simple resonant frequency or static displacement test. 


\begin{tabular}{|c|c|c|}
\hline Width of Beam & $\begin{array}{c}\text { Anchor Beam } \\
\text { Peak Amplitude in } \mu m\end{array}$ & $\begin{array}{c}\text { Corner Beam } \\
\text { Peak Amplitude in } \mu m\end{array}$ \\
\hline 1 & 11.11 & 11.28 \\
1.3 & 10.92 & 11.03 \\
1.6 & 10.66 & 10.81 \\
1.9 & 10.4 & 10.4 \\
2.1 & 10.21 & 10.2 \\
2.4 & 9.92 & 9.95 \\
2.7 & 9.73 & 9.67 \\
\hline
\end{tabular}

Table 3.7: Amplitude variation with change of single beam width

\begin{tabular}{|c|c|c|c|}
\hline Position of particle & Resonant frequency & Static displacement & Detectable \\
\hline Under the Stage & 18598 & $4.79 \mathrm{e}-3$ & Yes \\
Under the Comb & 19065 & $2.037 \mathrm{e}-2$ & Yes \\
Under the Beam & 2715.3 & 2.05 & No \\
\hline
\end{tabular}

Table 3.8: Particles under various parts of the device 
In a real time situation, more than one defect can occur at a time. For example, a beam which is over-etched, can have a particle under it, or it can be improperly released with portions still stuck to the substrate below. These kinds of combinations of defects can sometimes make the detection more difficult or sometimes easier. In this thesis the combined effects of etch variations and particles under the beam is considered.

As mentioned earlier, a point adhesion of the beam can be analyzed as an extra anchor to the beam [13]. Resonant frequency variation with the changes in both etch variation and the position of the particle under the beam are analyzed. The position of the adhesion point under the beam is referred to as the percentage of the beam length. For the anchor beam, $0 \%$ is the anchor end

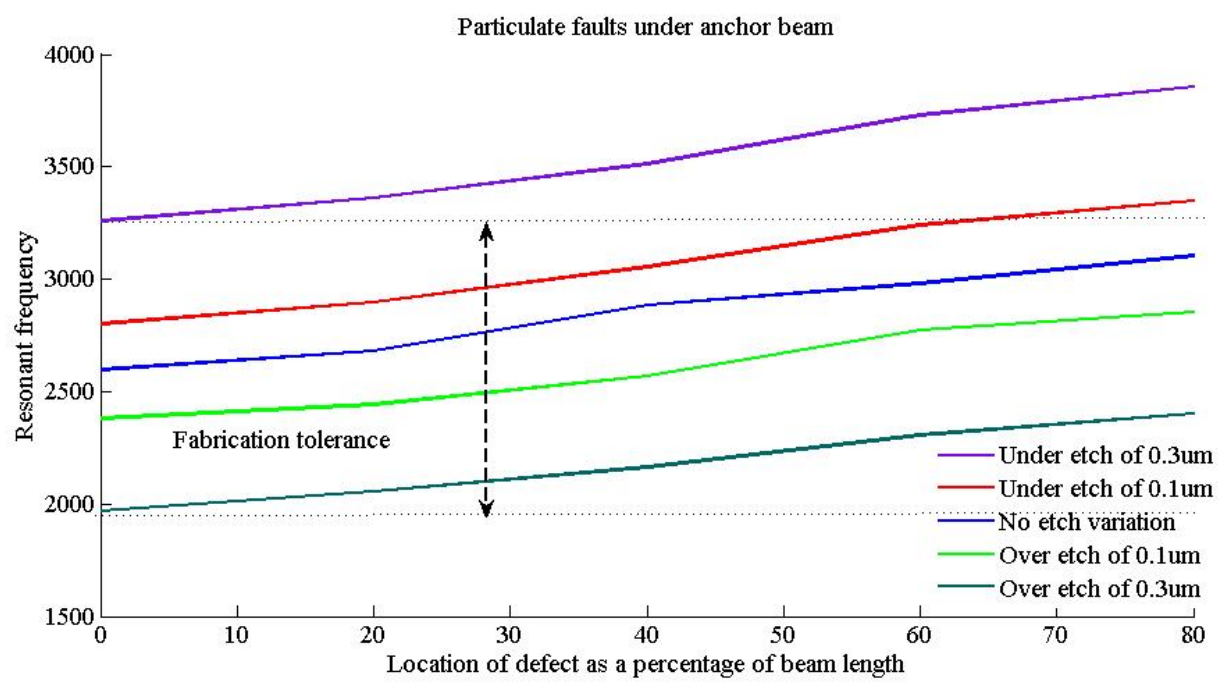

Figure 3.15: Resonant frequency variation due to multiple faults on anchor beam

and $100 \%$ is the truss end, for the corner beam, $0 \%$ is the truss end and $100 \%$ is the stage end. As concluded in $[13,27,28]$, the following figures show that resonant frequency alone cannot be used for fault detection. As can be observed from these figures, any amount of over-etch within the fabrication tolerances goes undetected by the resonant frequency test. From Fig.3.16 it can be observed that under-etch of the corner beam can be easily detected with the resonant frequency, but from the Fig.3.15 it can be noted that, for $0.1 \mu m$ under-etch (5\%), irrespective 
of the position, the defect is not detected. It can also be observed from the same figure that any amount of under-etch over $0.3 \mu \mathrm{m}(15 \%)$ is detectable with the resonant frequency test.

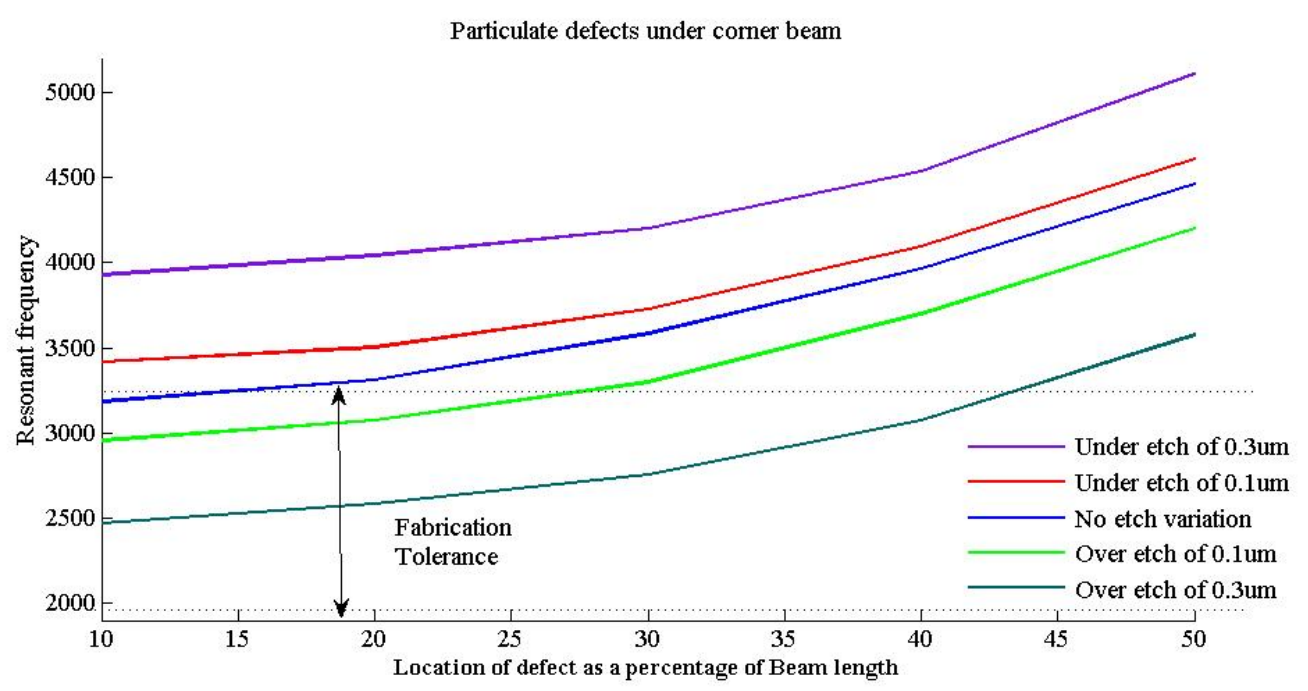

Figure 3.16: Resonant frequency variation due to multiple faults on corner beam

Though some defects are detectable, most go undetected when only resonant frequency is used as a detection parameter. Therefore these defects need to be detected by other means. In [28], an electrical method to detect these defects is presented. A differential amplifier is used to detect asymmetries of the device [an accelerometer], but this requires individual excitation of the fingers and also an electrical detection system introduces another level of complexity to the system. Also the device needs to be taken offline before conducting the testing. A high reliability device ultimately requires continuous in situ lifetime monitoring. A decoupled measurement system gives this flexibility. The detection method proposed here obtains in situ device positional state measurements through a decoupled optical system.

The ends of the flexures will be optically probed as a means to determine the presence of defects. The difference in the displacements at the ends of the flexures, is believed to give an indication of these defects. To verify this concept, initially the difference in the displacements of the flexures was analyzed using ANSYS at three frequencies:

1. At the resonant frequency of the defect free device 
2. At the Lower $3 \mathrm{~dB}$ frequency of the defect free device

3. At the Upper $3 \mathrm{~dB}$ frequency of the defect free device.

This was analyzed for both the anchor and corner beams. From Fig.3.17 it can observed that

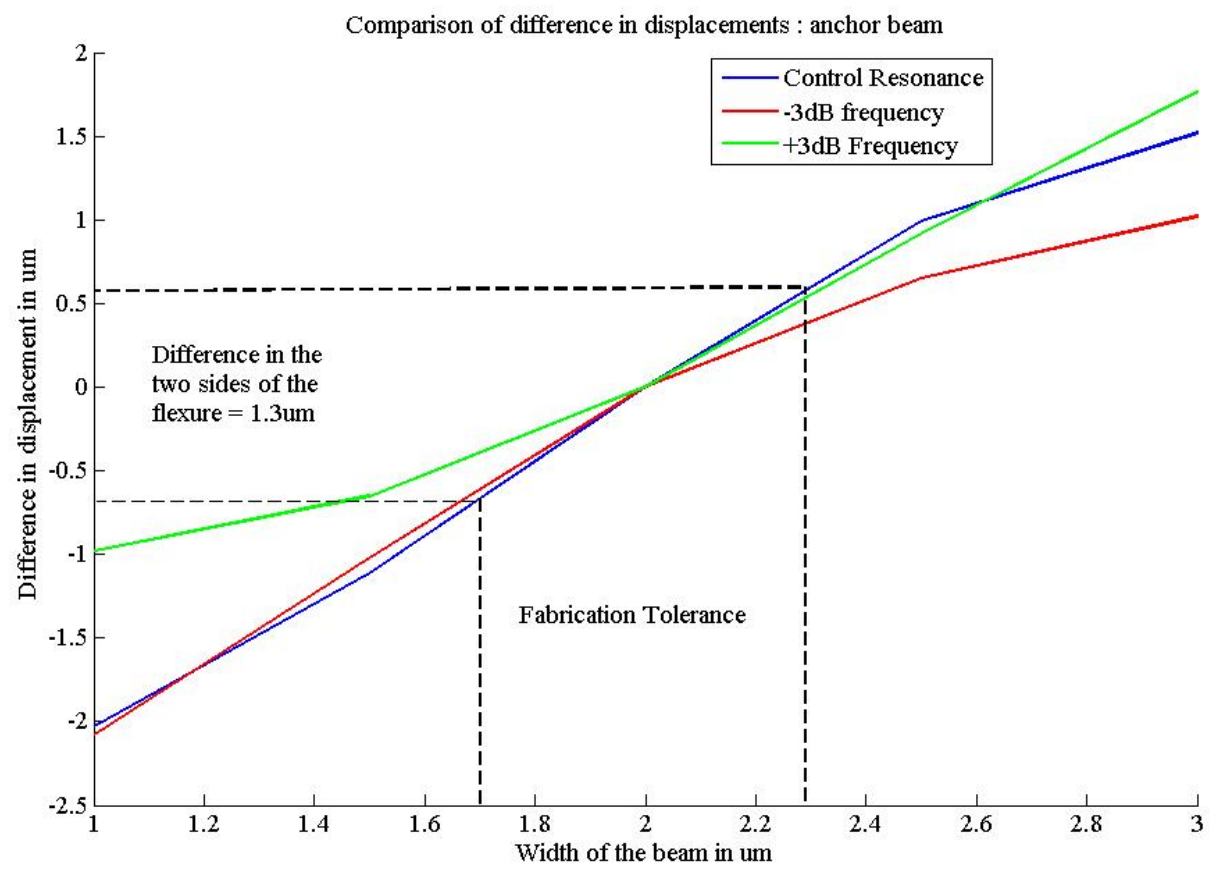

Figure 3.17: Difference in displacements at the ends of the flexure with variation of anchor beam width

there is a total difference in displacement of $0.8 \mu \mathrm{m}$, when the width of a single anchor beam is varied within the fabrication tolerance. From Fig.3.18 it can be observed that the difference in the displacements is about $1.3 \mu \mathrm{m}$, in the case of a corner beam. From both the graphs it can also be noted that this variation in difference is nearly maximum at the defect free device's resonant frequency.

The case of multiple faults was also analyzed using the displacements at the ends of the flexures. The under-etched cases which were undetected with a resonant frequency as shown in Fig.3.15 and Fig.3.16 can be detected using displacements at the ends of the flexures. This is clearly illustrated in the following figures. 


\begin{tabular}{|c|c|c|c|}
\hline Width of Anchor Beam & -3dB Frequency & Control Resonance & $+3 \mathrm{~dB}$ Frequency \\
\hline 1.3 & 1.42 & 1.38 & 0.8 \\
1.7 & 0.5 & 0.69 & 0.42 \\
1.9 & 0.18 & 0.24 & 0.16 \\
2.0 & 0 & 0 & 0 \\
2.1 & -0.16 & -0.23 & -0.16 \\
2.3 & -0.44 & -0.68 & -0.54 \\
2.7 & -0.84 & -1.35 & -1.36 \\
\hline
\end{tabular}

Table 3.9: Difference in displacement at ends of flexures with anchor beam width change

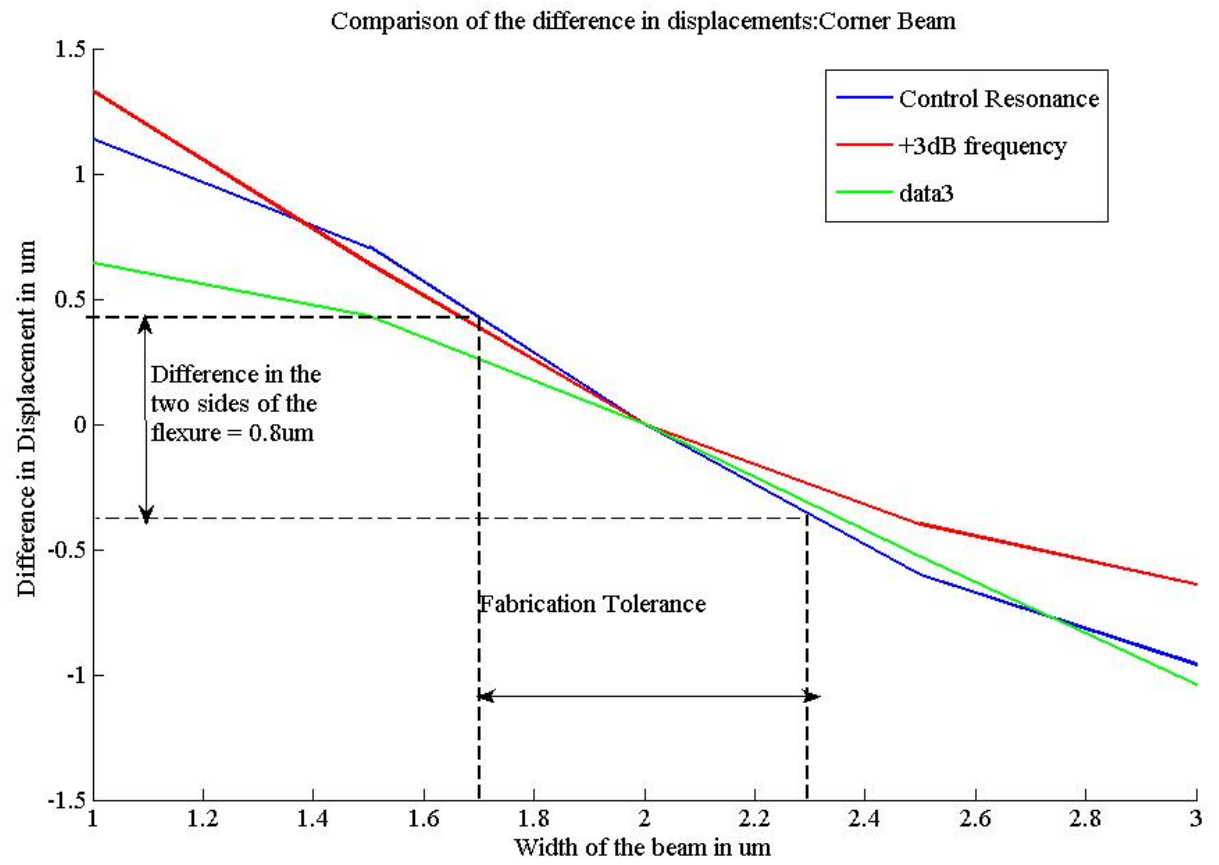

Figure 3.18: Difference in displacements at the ends of the flexure with variation of corner beam width

As seen in the figures 3.19 and 3.20 the difference in the displacement is maximum when the point of adhesion is near the beam and truss junction. As the point moves towards the stage, 


\begin{tabular}{|c|c|c|c|}
\hline Width of Corner Beam & $-3 \mathrm{~dB}$ Frequency & Control Resonance & +3dB Frequency \\
\hline 1.3 & -0.92 & -0.88 & -0.51 \\
1.7 & -0.34 & -0.43 & -0.26 \\
1.9 & -0.11 & -0.43 & -0.09 \\
2.0 & 0 & 0 & 0 \\
2.1 & 0.1 & 0.14 & 0.11 \\
2.3 & 0.32 & 0.41 & 0.28 \\
2.7 & 0.51 & 0.81 & 0.79 \\
\hline
\end{tabular}

Table 3.10: Difference in displacement at ends of flexures with Corner beam width change

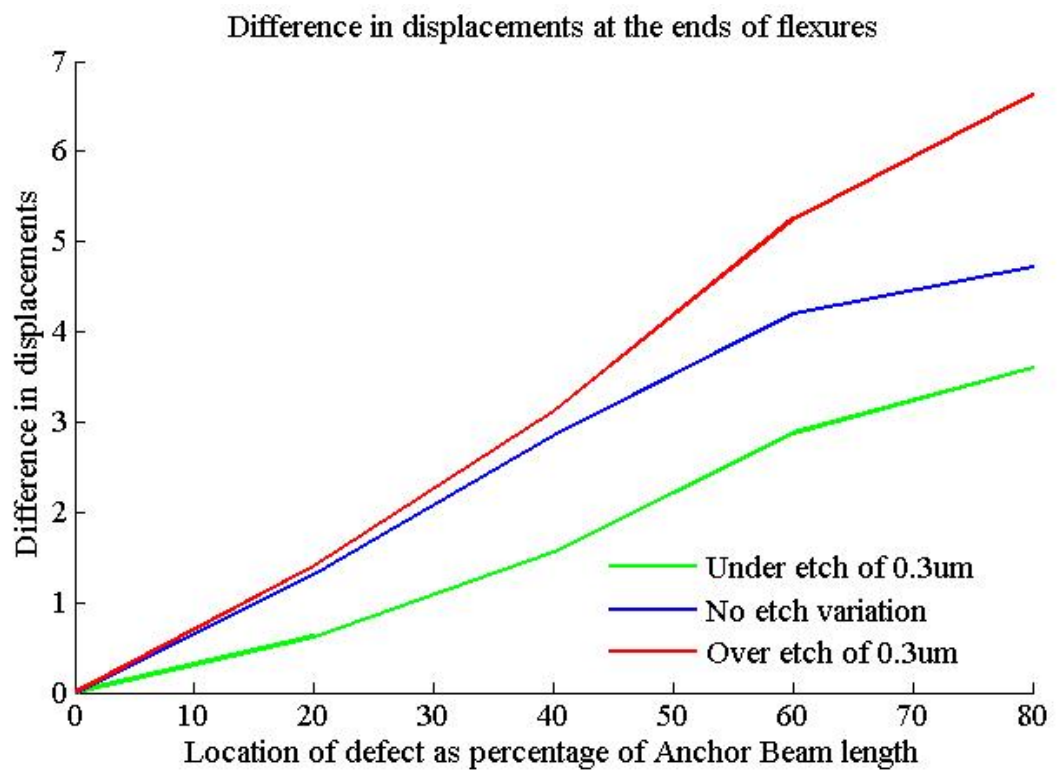

Figure 3.19: Difference in displacements at the ends of the flexure under multiple faults:Anchor Beam

the difference in the displacements decreases, but these faults can be detected by a simple static displacement or resonant frequency test. Hence with the appropriate optical characterization determination the displacements at the ends of the flexures can be done and thus this gives the ability to detect these defects with a decoupled optical measurement system. 


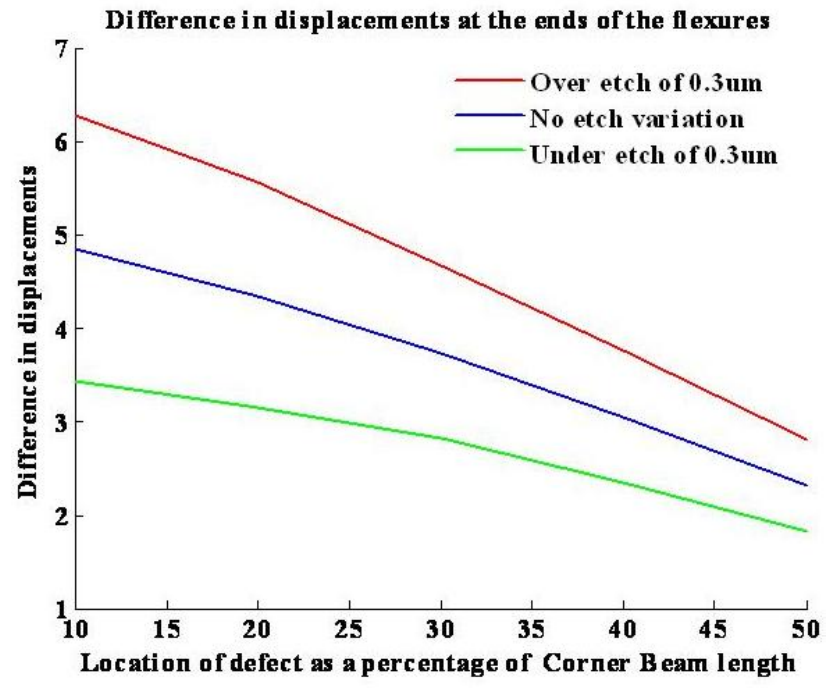

Figure 3.20: Difference in displacements at the ends of the flexure under multiple faults:Corner Beam 


\section{Chapter 4}

\section{MEMS Device design and testing}

This chapter deals with the design of the MEMS die in section 4.1 and processing of the same, before it can be used for testing, is described in section 4.2

\subsection{MEMS Layout Design}

Here experimental verification of the key simulation results is provided. Reliably generating these faults for these experiments poses problems as very small particles such as dust, vibrations due to the movement of the released die, testing equipment, fabrication tolerances etc. and the underlying fact that occurrence of these defects are statistical in nature. Therefore design features to emulate these faults are purposefully included.

The chip layout that was to be sent for MUMPS fabrication, was designed to have devices which emulate the following:

1. Control devices

2. Point adhesion of flexure beams at different locations.

3. Over and under etched beams.

4. Over and under etched beams with point adhesion of flexure beams at different locations.

5. Mass change devices for control purposes.

6. Four stator devices for asymmetrical electrical actuation. 


\begin{tabular}{|c|c|c|c|}
\hline $\begin{array}{c}\text { Name of the } \\
\text { device }\end{array}$ & Description & $\begin{array}{c}\text { Name of the } \\
\text { device }\end{array}$ & Description \\
\hline $400 \mathrm{C}$ & $\begin{array}{l}\text { Control Device with } \\
\text { flexure length } 400 \mu m\end{array}$ & $10 \mathrm{~A}$ & $\begin{array}{c}\text { Anchor beam stuck at } \\
10 \% \text { of its length }\end{array}$ \\
\hline $450 \mathrm{C}$ & $\begin{array}{l}\text { Control Device with } \\
\text { flexure length } 450 \mu \mathrm{m}\end{array}$ & $10 \mathrm{~A} 3$ & $\begin{array}{l}\text { Anchor beam stuck at } 10 \% \text { of its } \\
\text { length, with one beam width } 3 \mu \mathrm{m}\end{array}$ \\
\hline $\mathrm{T} 450$ & $\begin{array}{l}\text { Control Device with flexure length } \\
450 \mu \mathrm{m} \text { and beam width } 3 \mu \mathrm{m}\end{array}$ & A3 & One anchor beam width $3 \mu m$ \\
\hline $\mathrm{T} 10 \mathrm{C}$ & $\begin{array}{c}\text { Corner beam stuck at } 10 \% \\
\text { of its length }\end{array}$ & $\mathrm{C} 3$ & One corner beam width $3 \mu \mathrm{m}$ \\
\hline $\mathrm{T} 10 \mathrm{C} 2$ & $\begin{array}{l}\text { Corner beam stuck at } 10 \% \text { of its } \\
\text { length, with one beam width } 2 \mu \mathrm{m}\end{array}$ & M10 & $10 \%$ mass change \\
\hline $\mathrm{T} 20 \mathrm{~A}$ & $\begin{array}{l}\text { Anchor beam stuck at } 20 \% \\
\text { of its length }\end{array}$ & M5 & $5 \%$ mass change \\
\hline $\mathrm{T} 50 \mathrm{~A}$ & $\begin{array}{l}\text { Anchor beam stuck at } 50 \% \\
\text { of its length }\end{array}$ & 4 Stators & $\begin{array}{l}\text { Stator split in the middle } \\
\text { to give } 4 \text { stators }\end{array}$ \\
\hline T50A2 & $\begin{array}{l}\text { Anchor beam stuck at } 50 \% \text { of its } \\
\text { length, with one beam width } 2 \mu \mathrm{m}\end{array}$ & & \\
\hline
\end{tabular}

Table 4.1: Devices in the present layout

The control devices have a beam width of $2 \mu \mathrm{m}$, which is the minimum feature size achievable with MUMPS [11]. Therefore to design the over-etching of the device, a control device with all the beam widths of $3 \mu \mathrm{m}$, and another device with one of the beams having a width of $2 \mu \mathrm{m}$ was designed. Anchor and corner beams stuck at different locations and over-etched devices stuck at similar locations were also designed. Table 4.1 gives the devices that were designed for the present layout. The MUMPS chip layout is on a $1 \mathrm{cmX} 1 \mathrm{~cm}$ die. The effective area in which the devices are laid out is $0.8 \mathrm{cmX} 0.8 \mathrm{~cm}$. A small amount of added featureless area is included around the die perimeter to accommodate loading the die in the cage of the critical release dryer. 


\begin{tabular}{|c|c|c|c|c|c|c|c|c|c|}
\hline \multirow[t]{2}{*}{ Device } & \multicolumn{3}{|c|}{$\begin{array}{c}\text { Mass } \\
\text { e-10 Kg }\end{array}$} & \multicolumn{3}{|c|}{$\begin{array}{c}\text { Spring } \\
\text { Constant(N/m) }\end{array}$} & \multicolumn{3}{|c|}{$\begin{array}{c}\text { Resonant } \\
\text { Frequency }(\mathrm{Hz})\end{array}$} \\
\hline & Min & Std & Max & Min & Std & Max & Min & Std & Max \\
\hline $\mathrm{T} 450$ & 2.065 & 2.083 & 2.101 & .126 & .181 & .249 & 3936.3 & 4692.5 & 5485.5 \\
\hline $\mathrm{T} 20 \mathrm{~A}$ & 2.071 & 2.089 & 2.107 & .142 & .202 & .276 & 4174.7 & 4948.1 & 5758.9 \\
\hline T50A & 2.071 & 2.089 & 2.107 & .171 & .239 & .323 & 4569 & 5384.6 & 6238.3 \\
\hline $\mathrm{T} 10 \mathrm{C}$ & 2.071 & 2.089 & 2.107 & .227 & .317 & .428 & 5000 & 6203.6 & 7174.3 \\
\hline $10 \mathrm{~A}$ & 2.012 & 2.029 & 2.047 & .033 & .056 & .090 & 2034.3 & 2662.9 & 3340.4 \\
\hline T50A2 & 2.064 & 2.082 & 2.099 & .144 & .209 & .290 & 4214.7 & 5049.5 & 5916.9 \\
\hline $\mathrm{T} 10 \mathrm{C} 2$ & 2.064 & 2.082 & 2.099 & .154 & .222 & ..308 & 4346 & 5199.8 & 6102.3 \\
\hline $10 \mathrm{~A} 3$ & 2.018 & 2.036 & 2.054 & .039 & .066 & .102 & 2238.6 & 2872.8 & 3554.1 \\
\hline $\mathrm{A} 3$ & 2.013 & 2.031 & 2.049 & .037 & .063 & .097 & 2182.9 & 2803.8 & 3472.9 \\
\hline C3 & 2.013 & 2.031 & 2.049 & .035 & .061 & .097 & 2110.7 & 2769.5 & 3473 \\
\hline 4Stators & 2.065 & 2.083 & 2.101 & .053 & .053 & .054 & 1942 & 2555.4 & 3216.6 \\
\hline
\end{tabular}

Table 4.2: Range of parameters due to fabrication tolerance: Current Die

The final layout of the die is shown in Fig.4.1 and the layout of the defects described above is shown in Figs.4.2,4.3,4.4,4.5. The mass, spring constant and resonant frequency ranges due to fabrication tolerances, for each of the devices under consideration in the current die layout, are given in table 4.2 


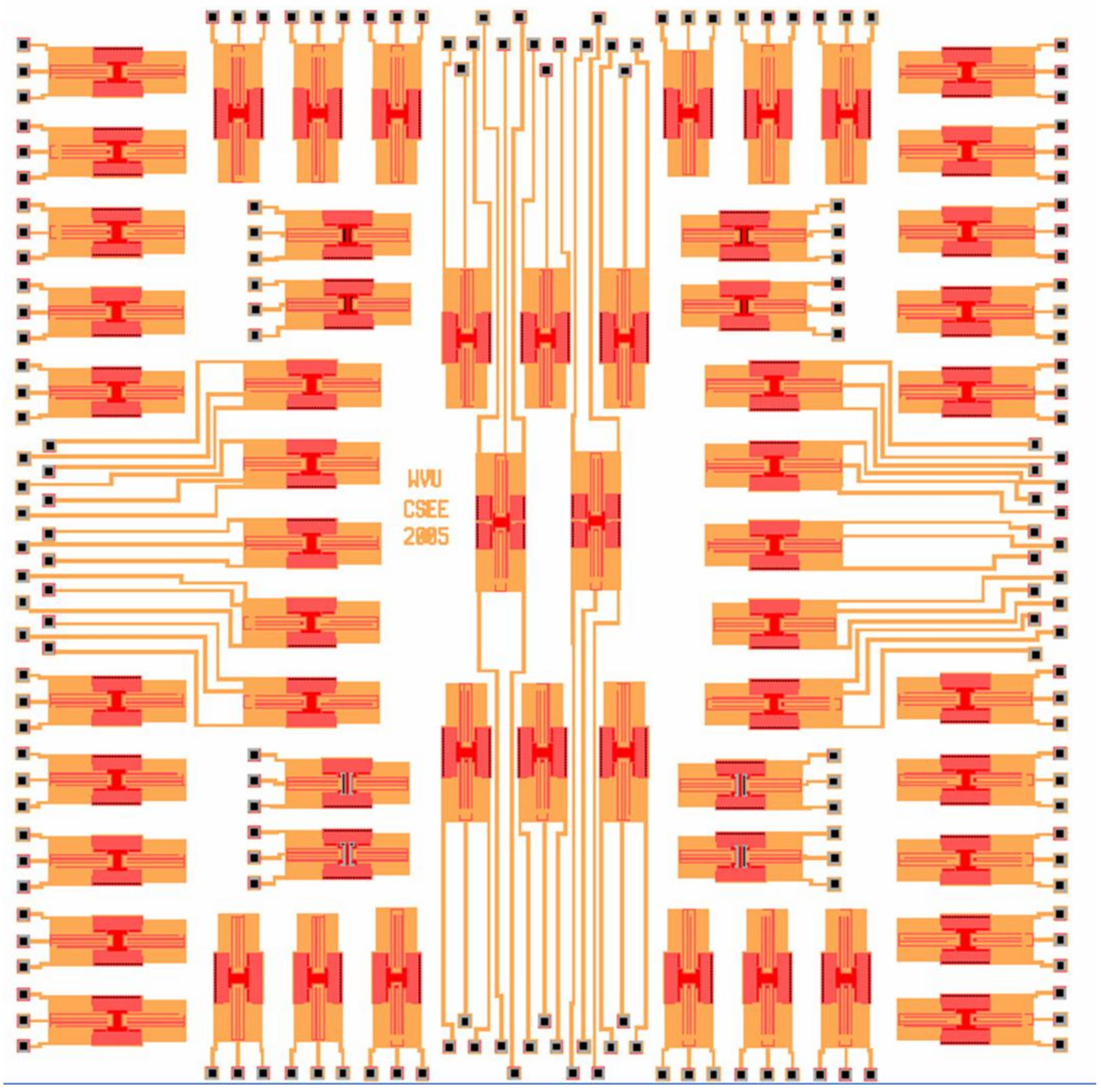

Figure 4.1: Final Die Layout 


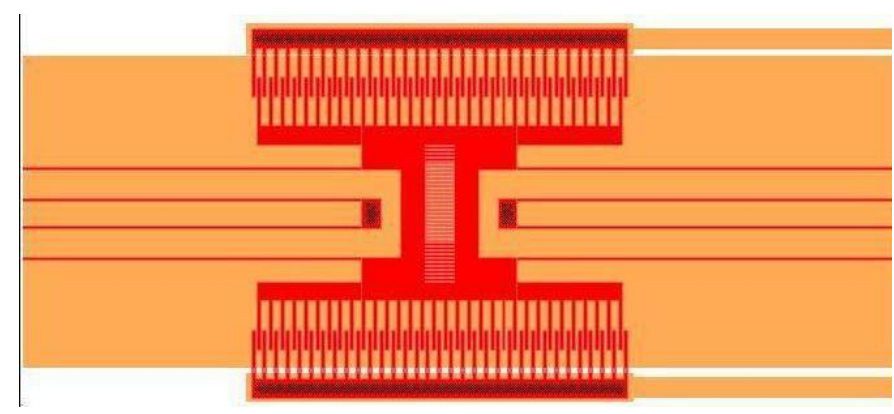

(a) Control Device

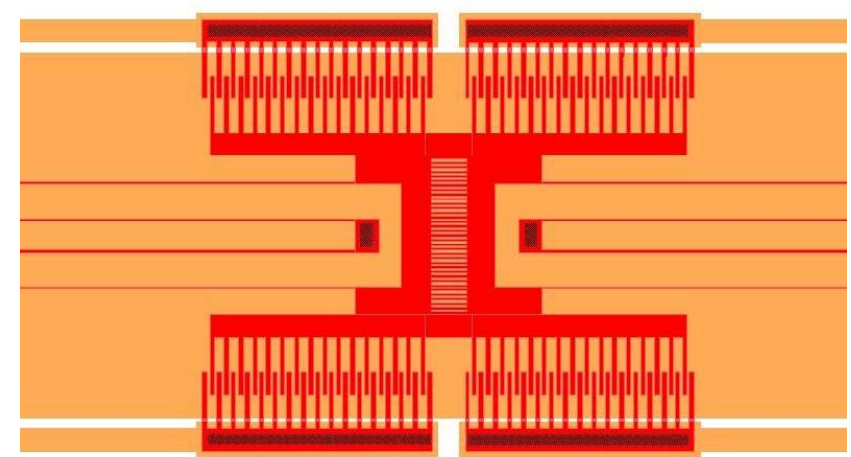

(b) Four Stator Device

Figure 4.2: Devices

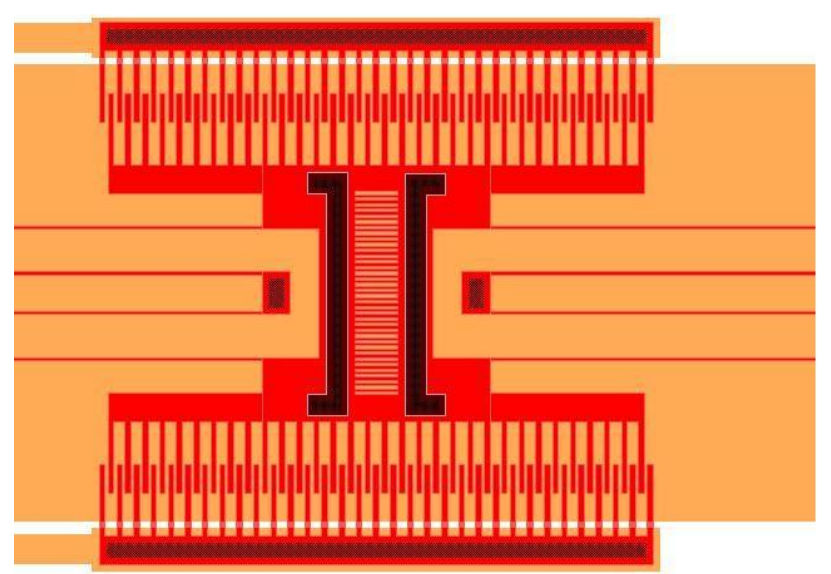

(a) Mass Change Device

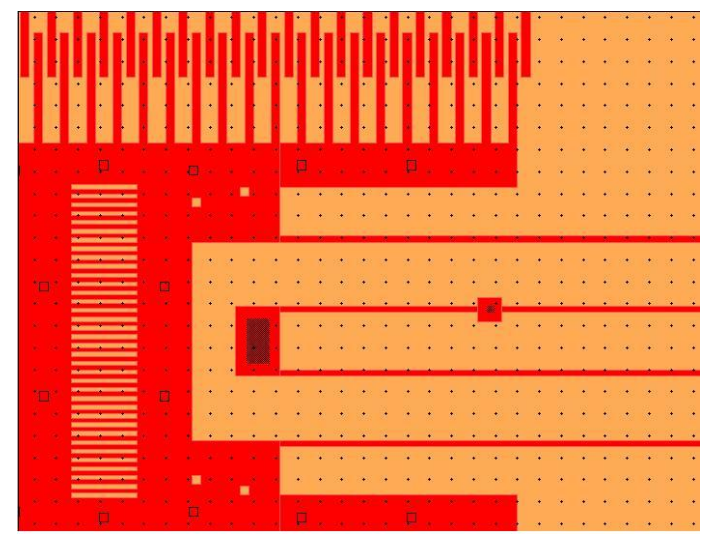

(b) Point of adhesion under Anchor Beam

Figure 4.3: Devices 


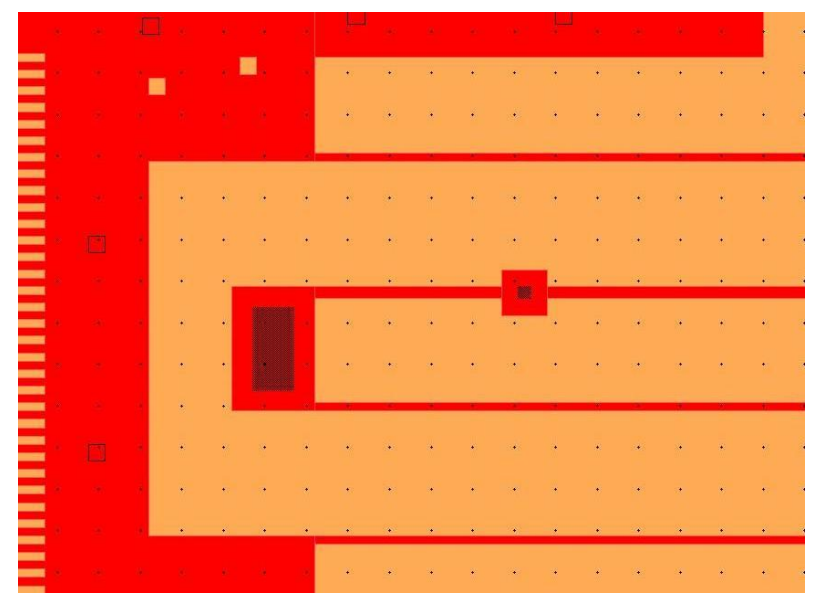

(a) Anchor beam with point of adhesion and under-etch

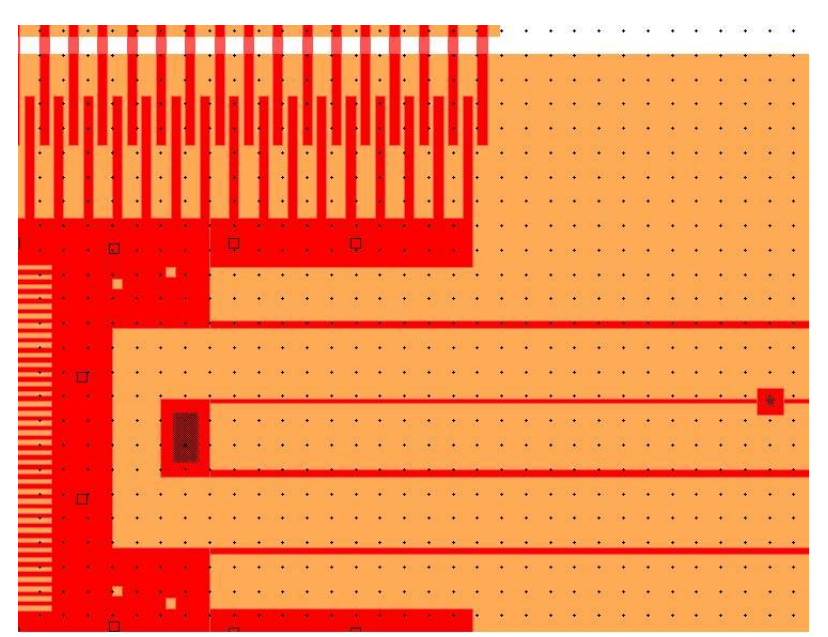

(b) Anchor Beam with point of adhesion and over-etc

Figure 4.4: Multiple Faults

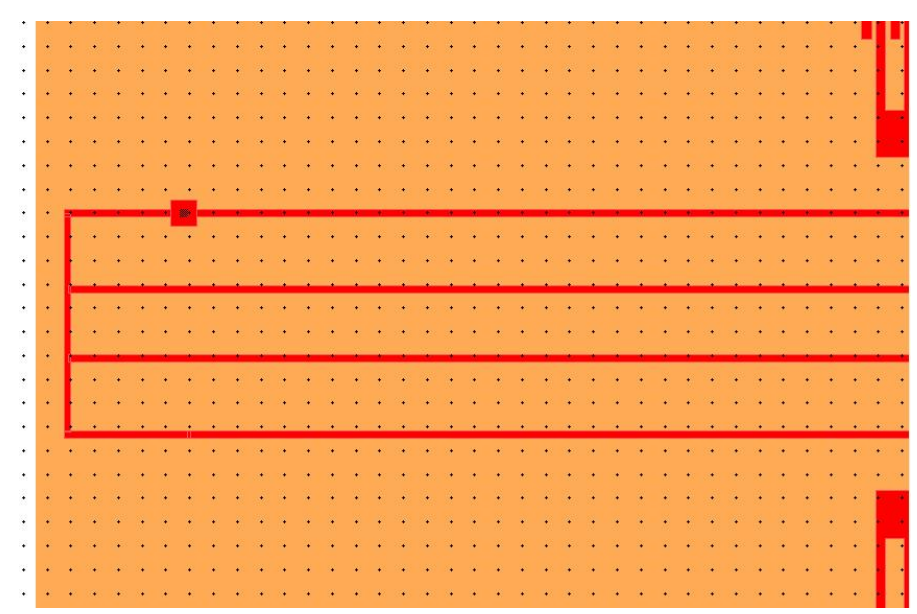

(a)Point of adhesion under Corner Beam

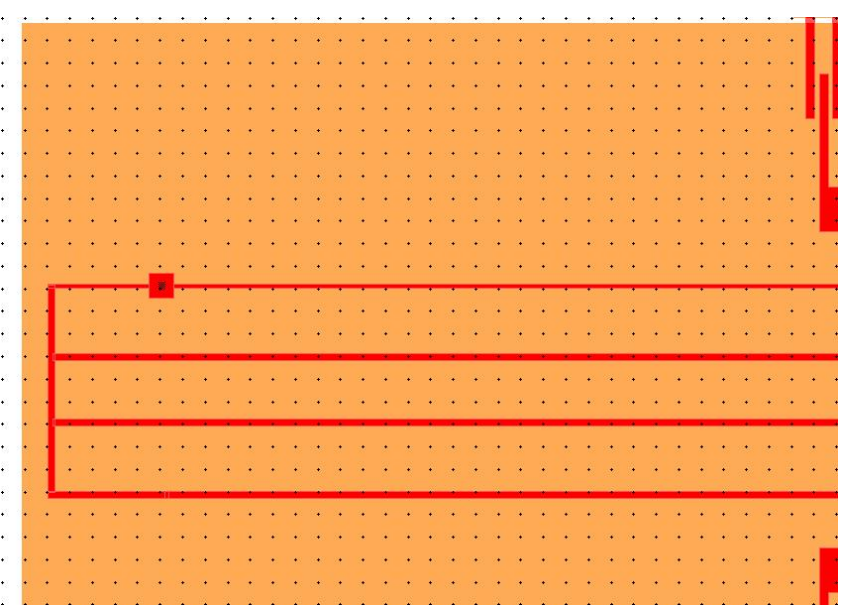

(b) Corner Beam with point of adhesion and over-etch

Figure 4.5: Multiple Faults 
As mentioned earlier, to emulate the effect of over-etching of devices, a device with the width of the beams $3 \mu \mathrm{m}$ was designed. The plots $3.15,3.16,3.19$ and 3.20 which were simulated for

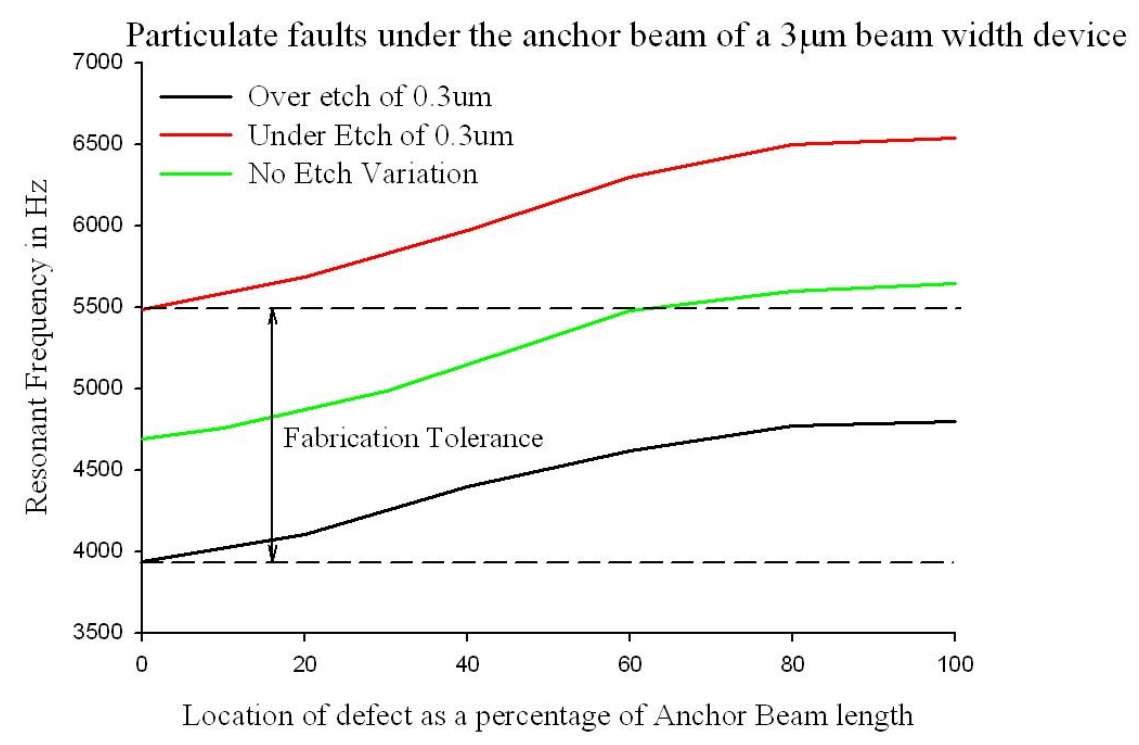

Figure 4.6: Resonant frequency variation due to multiple faults on anchor beam of a $3 \mu \mathrm{m}$ beam width device

a $2 \mu \mathrm{m}$ width beams are re-simulated to understand the behavior of the $3 \mu \mathrm{m}$ beam width device under the conditions of over and under etch, and also to estimate the expected difference in the displacement at the ends of the flexures. Figures 4.6, 4.7, 4.8 and 4.9 show the simulation results for the detection of multiple faults of the control device with beam width of $3 \mu \mathrm{m}$. As most of the devices are designed with $3 \mu \mathrm{m}$ beam width, these plots give an estimate of the difference in the displacement when conducting experiments. From Fig.4.6 it can be noted that the case of over-etch combined with point of adhesion defects, causes defect masking. Fig.4.7, it can be observed that most of the cases can be detected by the resonant frequency test, but some of the over-etch cases come within the envelope of the fabrication tolerance. Also from Figs.4.8 and 4.9, it can be seen that the maximum difference in displacement for the anchor beam defective devices is about $2 \mu \mathrm{m}$ and for the corner beam it is about $3 \mu \mathrm{m}$. 


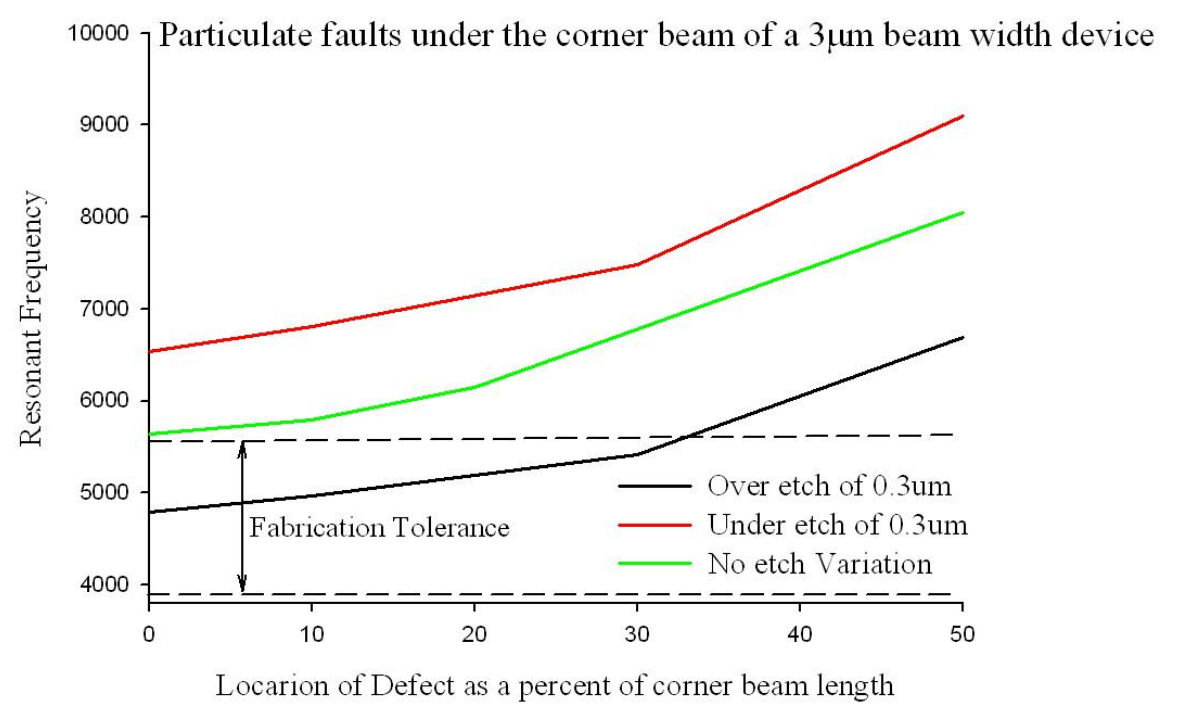

Figure 4.7: Resonant frequency variation due to multiple faults on corner beam of a $3 \mu \mathrm{m}$ beam width device

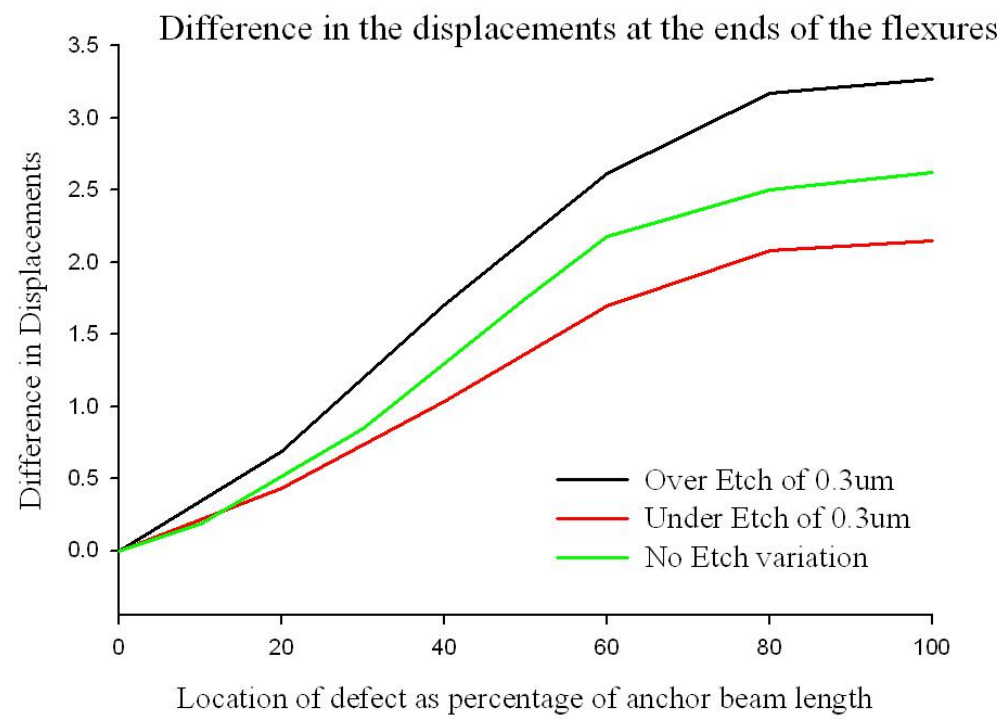

Figure 4.8: Difference in displacements at the ends of the flexure under multiple faults of a $3 \mu \mathrm{m}$ beam width device:Anchor Beam 


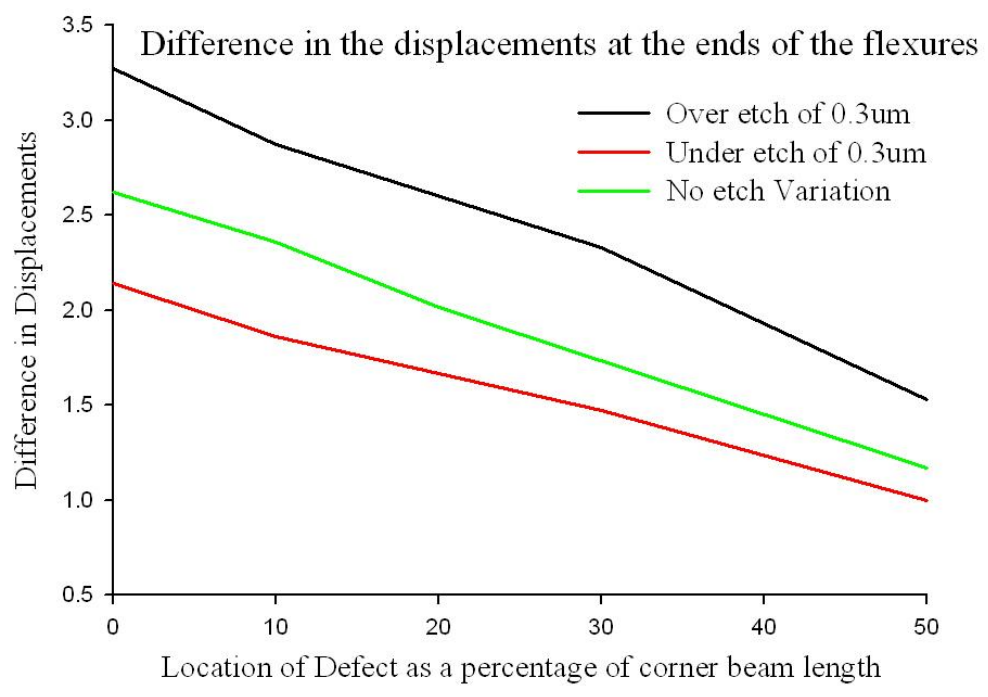

Figure 4.9: Difference in displacements at the ends of the flexure under multiple faults of a $3 \mu \mathrm{m}$ beam width device:Corner Beam

\subsection{Processing of the MEMS Die}

After the die is received it needs to be processed and tested to observe the behavior of the devices. The following subsections describe briefly the various steps involved.

\subsubsection{Polishing}

To enable through wafer optical testing, the backside of the wafer needs to be polished. This prevents scattering of the through wafer Laser and gives a signal with an improved signal to noise ratio. The die is mounted on a small stainless steel chuck with wax and polished on a polishing machine. For the initial polish a 6 micron diamond paste is used and for the finer polish a 1 micron diamond paste is used.

\subsubsection{Release}

After the polish, the die needs to be released. It is treated through a series of ultrasound agitations in acetone, to remove the coating of photoresist on the devices. It is then dipped in 


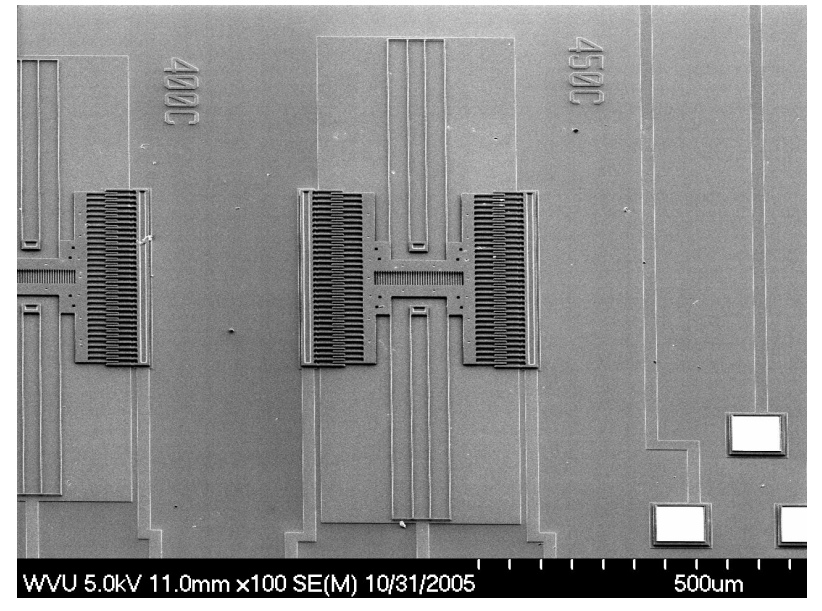

(a) Control Device

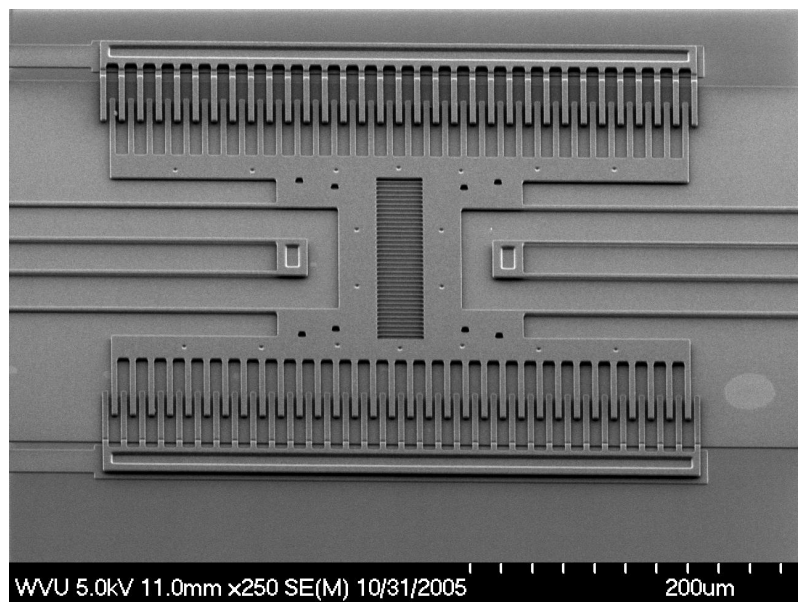

(b) Closeup:Stage

Figure 4.10: SEM Pictures

$49 \% \mathrm{HF}$, for about 2 mins and 15 seconds to etch away the silicon dioxide layer and release the microdevices.

\subsubsection{Drying}

The released die is then dried in a supercritical liquid $\mathrm{CO}_{2}$ dryer. This minimizes stiction forces acting on the die after the release. The supercritical $\mathrm{CO}_{2}$ dryer takes the die through a series of cooling and heating cycles, in the process replaces the alcohol, in which the die is submerged, with liquid $\mathrm{CO}_{2}$ and then sublimates the liquid. This gives us a dried die. It is critical that the alcohol used should be atleast $99.9 \%$ pure and the $\mathrm{CO}_{2}$ cylinder is a liquid $\mathrm{CO}_{2}$ bone dry grade cylinder with a syphon tube. The Figs.4.10,4.11,4.12,4.13 and 4.14 show the Scanning Electron Microscope pictures of a released die. They pictures show the released MEMS structures and also different defects that were designed.

\subsubsection{Fabrication of glass packages}

Before the release of the die, the glass package on which the die needs to be mounted, should be fabricated. The fabrication procedure as described in [4] was used to fabricate this package. 


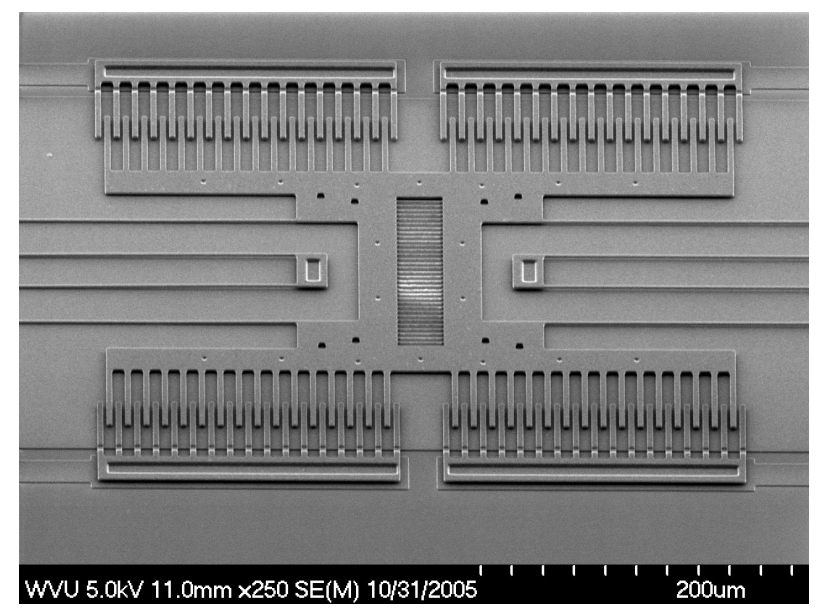

(a) 4 Stator Device for asymmetrical actuation

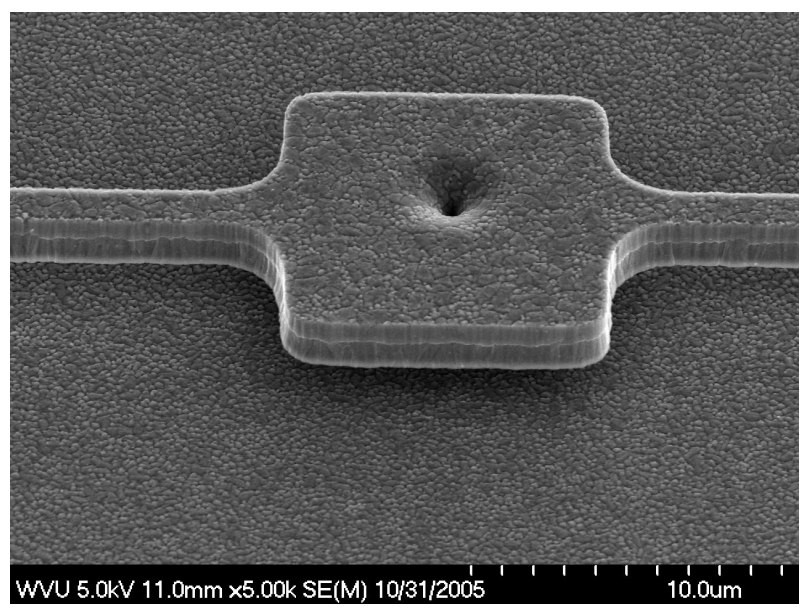

(b)Closeup:Point of adhesion

Figure 4.11: SEM Pictures

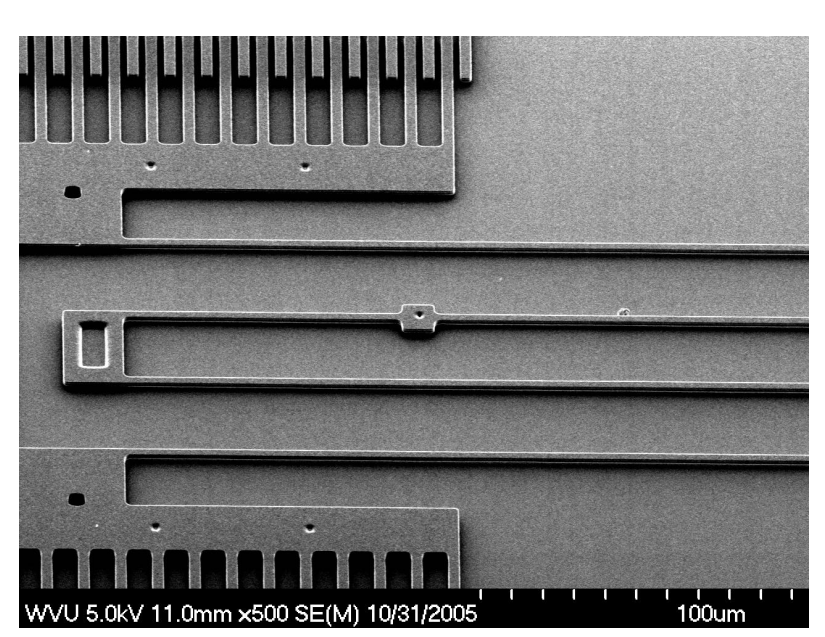

(a) Point of adhesion on anchor beam

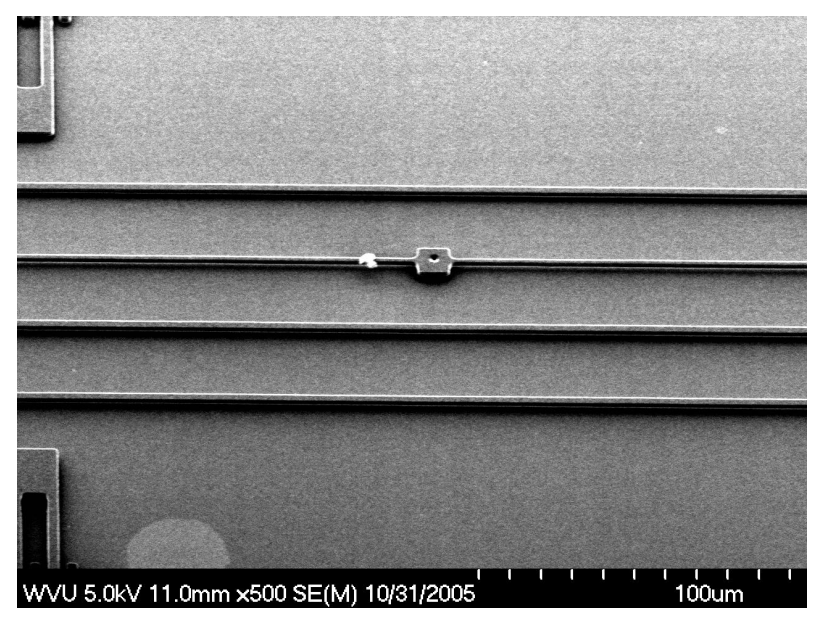

(b) Point of adhesion and over-etch on anchor beam

Figure 4.12: SEM Pictures 


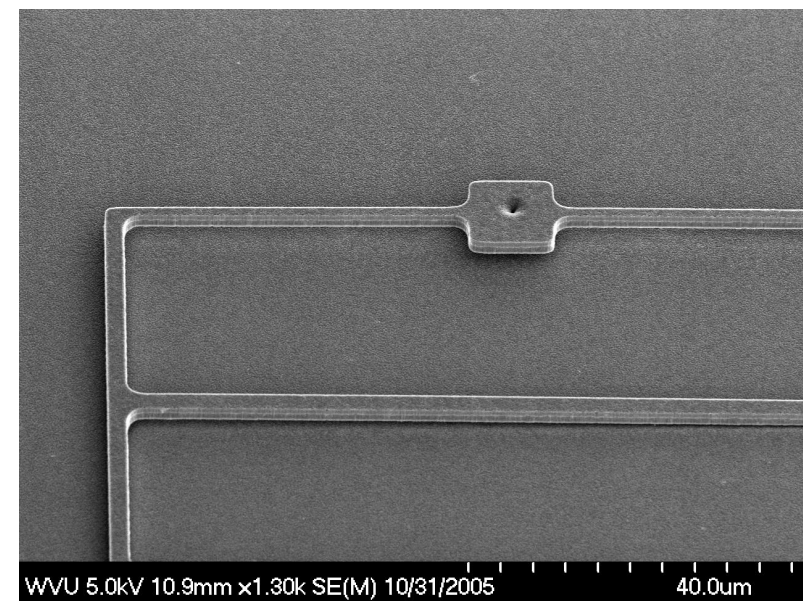

(a) Point of adhesion on corner beam

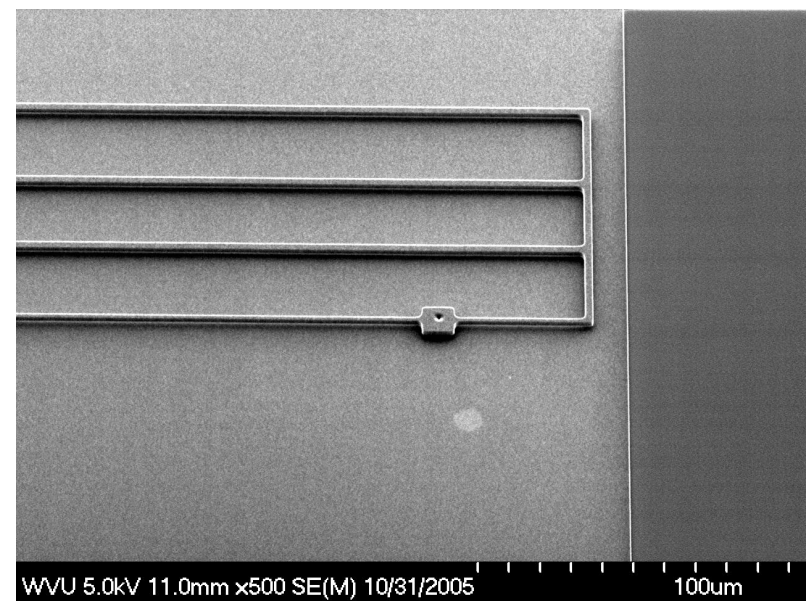

(b) Point of adhesion and over-etch on corner beam

Figure 4.13: SEM Pictures

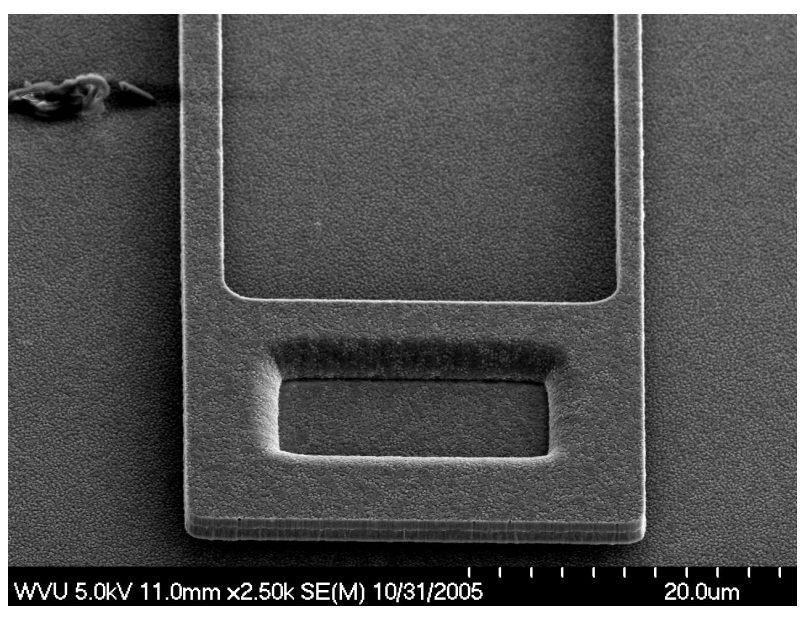

(a) The anchor beam width is over-etched by $1 \mu \mathrm{m}$

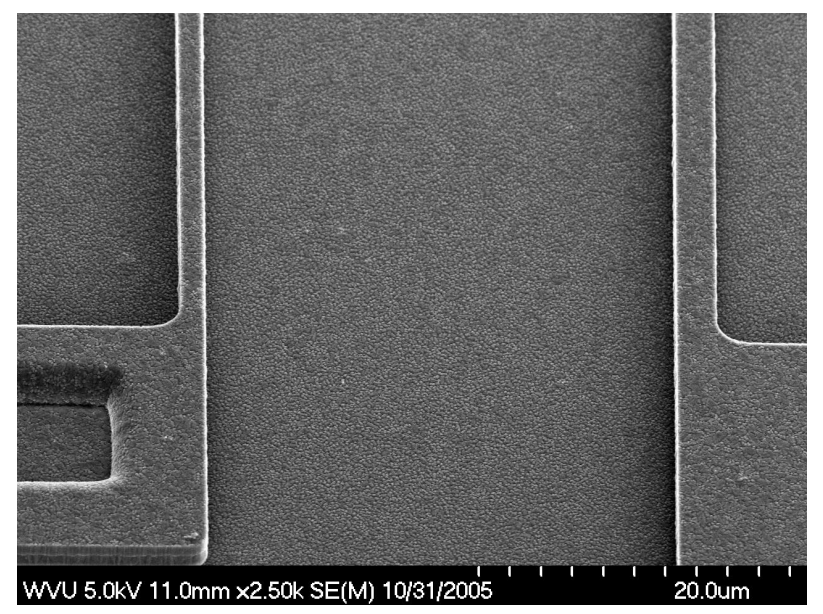

(b) The corner beam width is over-etched by $1 \mu \mathrm{m}$

Figure 4.14: SEM Pictures 
Some changes in the described process were done to optimize the package to the current need. The exposure time was varied from 100 seconds to 120 seconds(with UV power $1.9 \mathrm{~mW}$ ). But

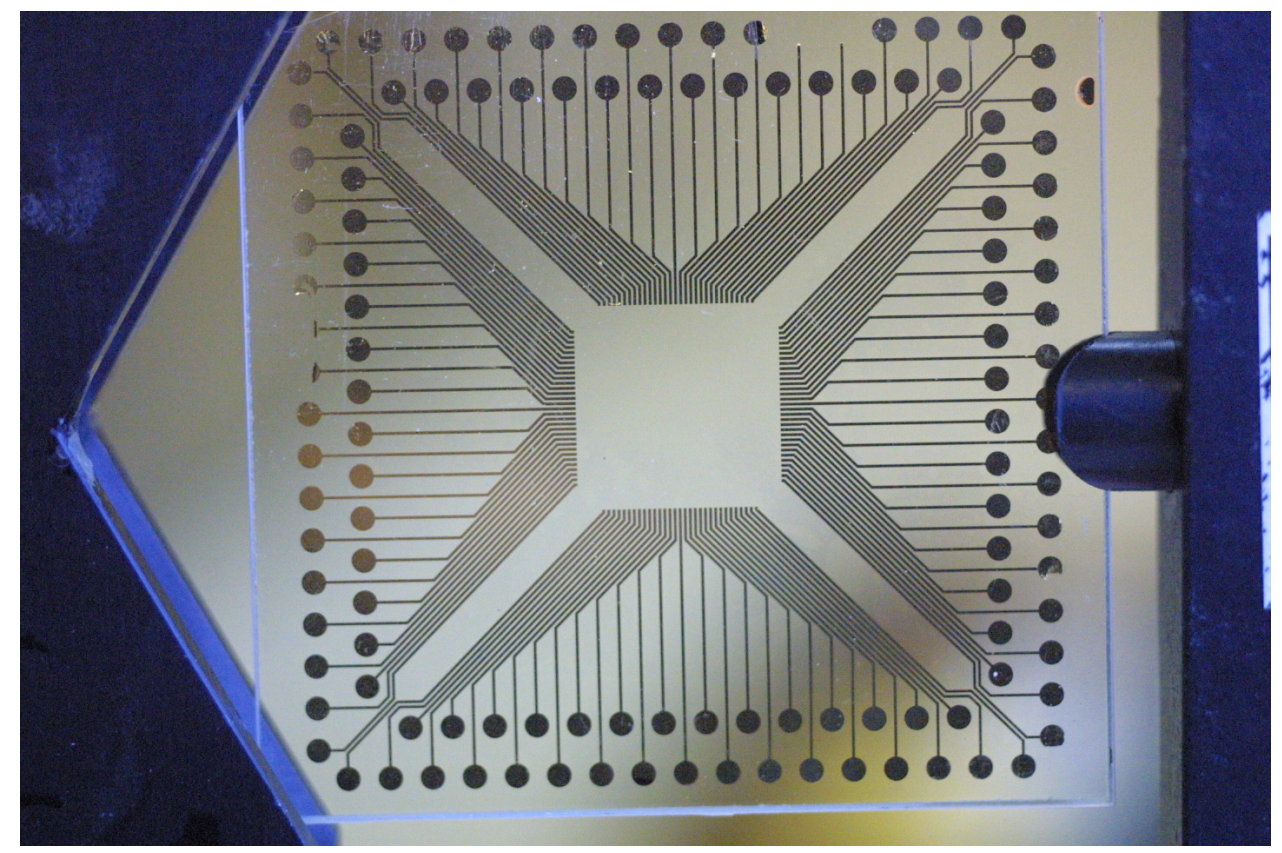

Figure 4.15: Fabricated Gold Package

this exposure time depends on the power of the UV source (here the power is taken without the mask) and hence needs to be estimated accordingly. Also instead of using the gold etchant 8148 , a lesser acidic version of the same etchant 8118 was used. This was done, because 8148 etchant was sometimes over-etching and wiping off the patterns. Though 8118 takes longer time to etch (about 30-35mins), it is safer to use. Also the photoresist was stripped by dipping the sample in acetone for 10 mins, instead of using the photoresist stripper. The pattern sizes were not effected by these changes. Fig.4.15 shows the fabricated package on the glass substrate.

\subsubsection{Bonding}

The die is mounted on the fabricated glass package with the help of epoxy and set aside for 4-5 hours. The die is then heated on the hot plate attached to the bonder to enable the bonding of the contact pads to the patterned gold package. Bonding the die this way, gives us access to the 


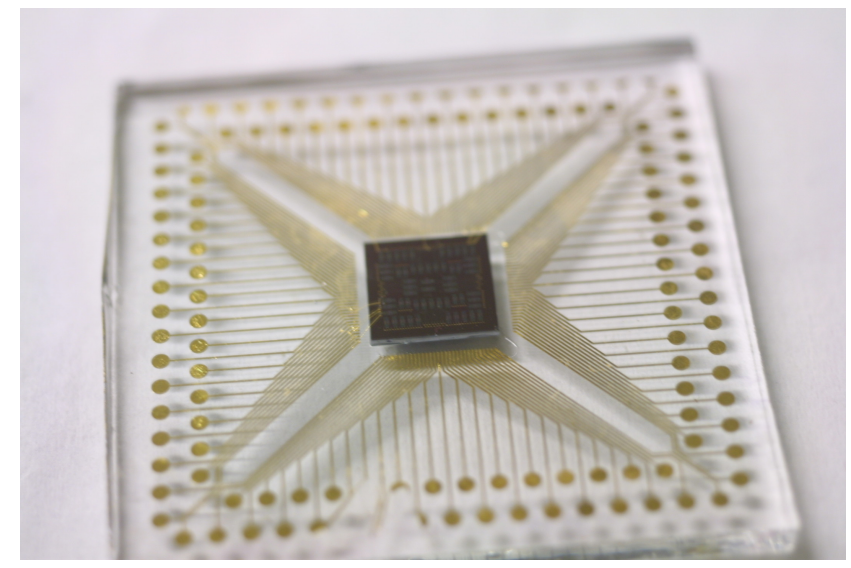

(a) Die on the fabricated glass substrate

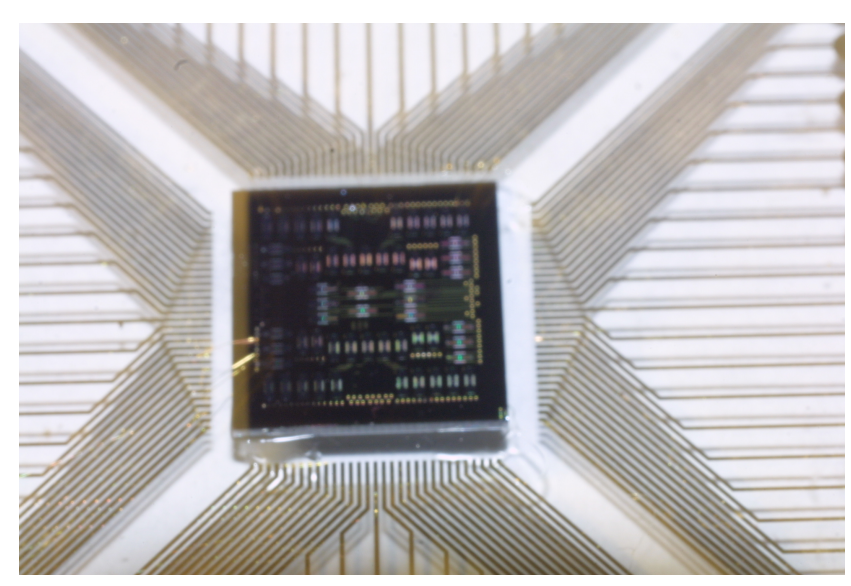

(b) Closer View

Figure 4.16: Bonded Die

devices, without direct contact with the device. This helps in integrated optical packaging of the die. Fig.4.16, shows the die bonded onto the fabricated glass substrate.

\subsubsection{Testing}

The die is then tested either under the through wafer system or under the vibrometer and the parameters of interest are collected and analyzed. This is explained in detail in Chapter 5. 


\section{Chapter 5}

\section{Experimental Results and Discussion}

In this chapter the experimental results are discussed. The optical data from the MEMS

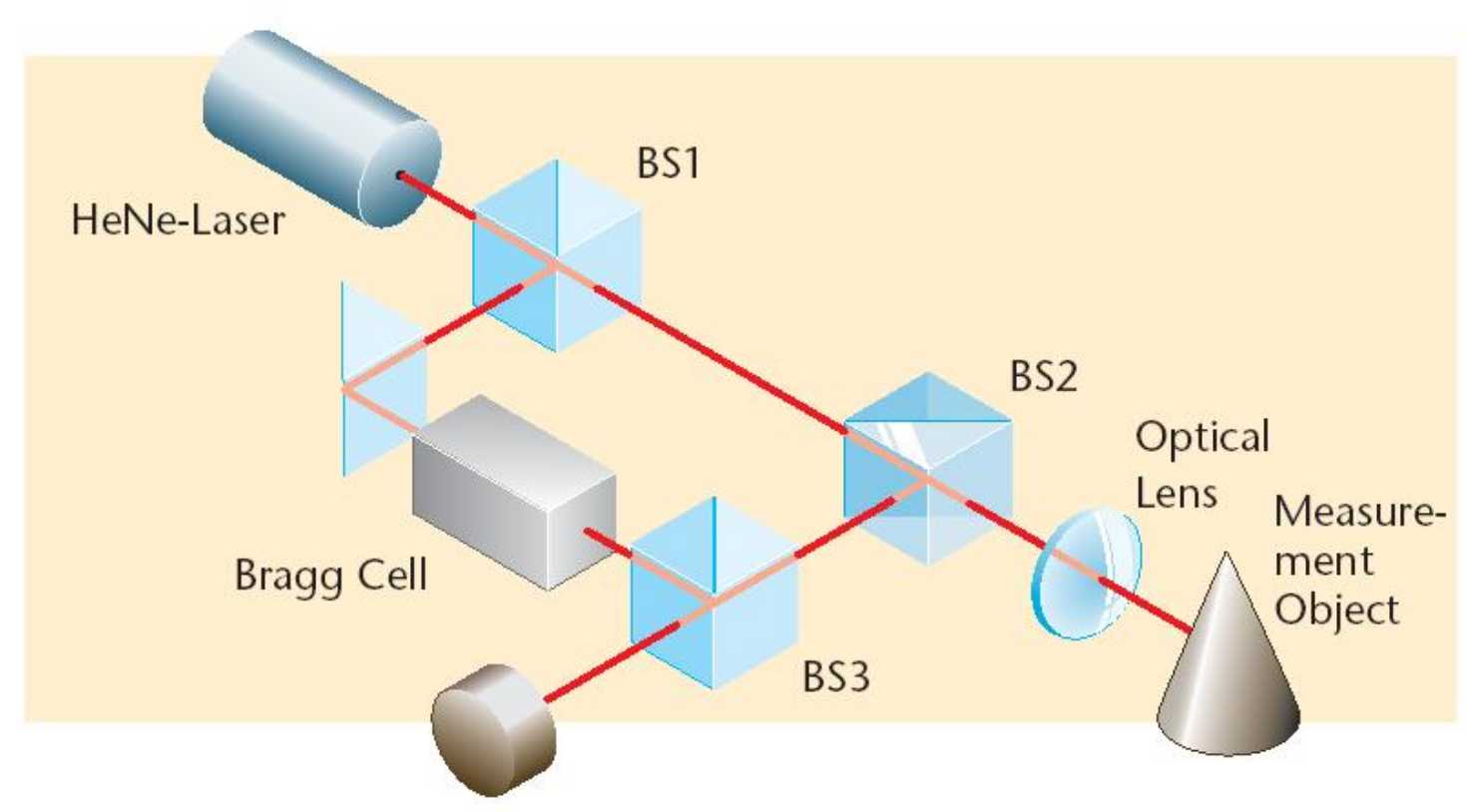

Figure 5.1: Basic Operation of the vibrometer ${ }^{1}$

devices which were released and tested, was taken and analyzed. It is shown that the fault detection of these devices is possible using the optical data. Also it is shown that the values

${ }^{1}$ Polytec User Manual 
obtained from testing are consistent with those predicted by the simulations described in Chapter 3.

\subsection{Optical Testing and Setup}

The released and bonded MEMS die is tested under the vibrometer. Fig.5.1 shows us the setup of the vibrometer. It works on the principle of doppler effect. A Laser beam is split up by the first beam splitter into two beams: measurement and reference. The measurement beam passes through the second beam splitter and is focussed through the objective of the microscope. This can be placed wherever measurement is required. A third beam splitter combines the two beams, and the sensor that captures these, gives the difference in the two beams.

The beam used here is a $633 \mathrm{~nm}$ wavelength device, with a spot size of about $2.5 \mu \mathrm{m}$. The optical signal obtained from the vibrometer alternates between a positive and negative value when the measurement beam moves between poly 0 and poly 1 layers as can be seen in Fig.5.2. The transitions give a modulation in the collected optical data, whose value is proportional to the

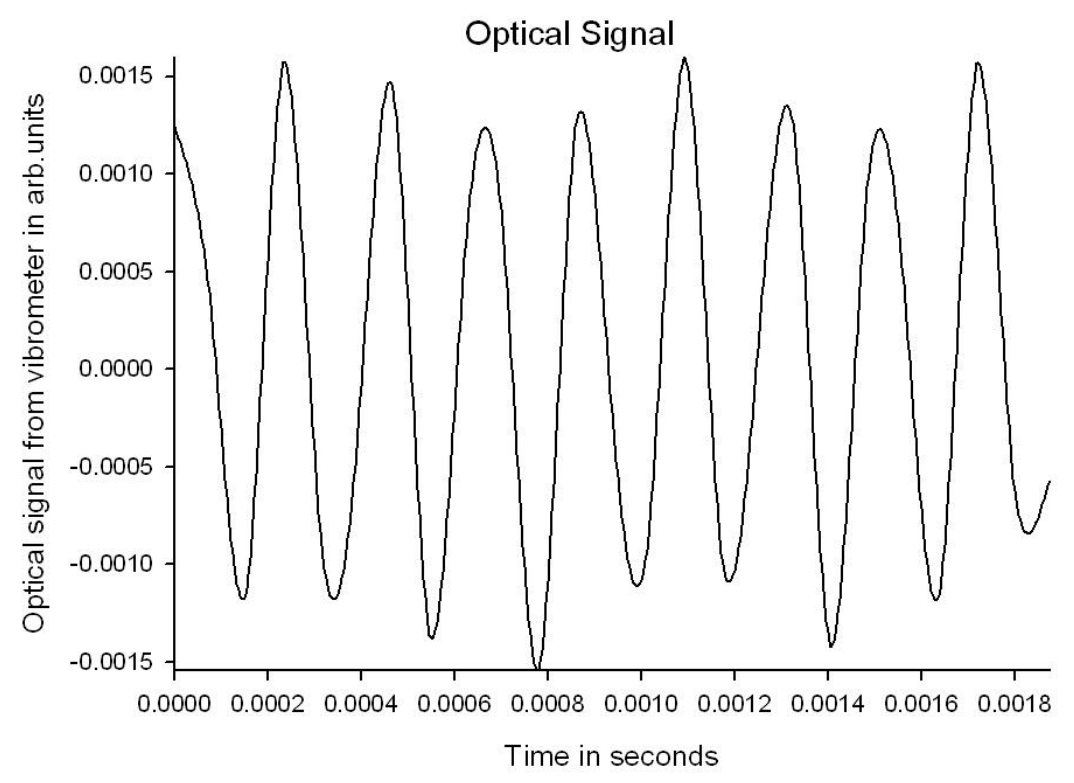

Figure 5.2: Optical signal from the vibrometer 
displacement of the system in the lateral direction. The device was probed at both the ends of the flexure separately and the difference in the signal was calculated. Also measurements were taken with the reference beam placed on one end of the flexure and the measurement beam placed on another end. This directly gave the resultant difference signal. These two methods of data

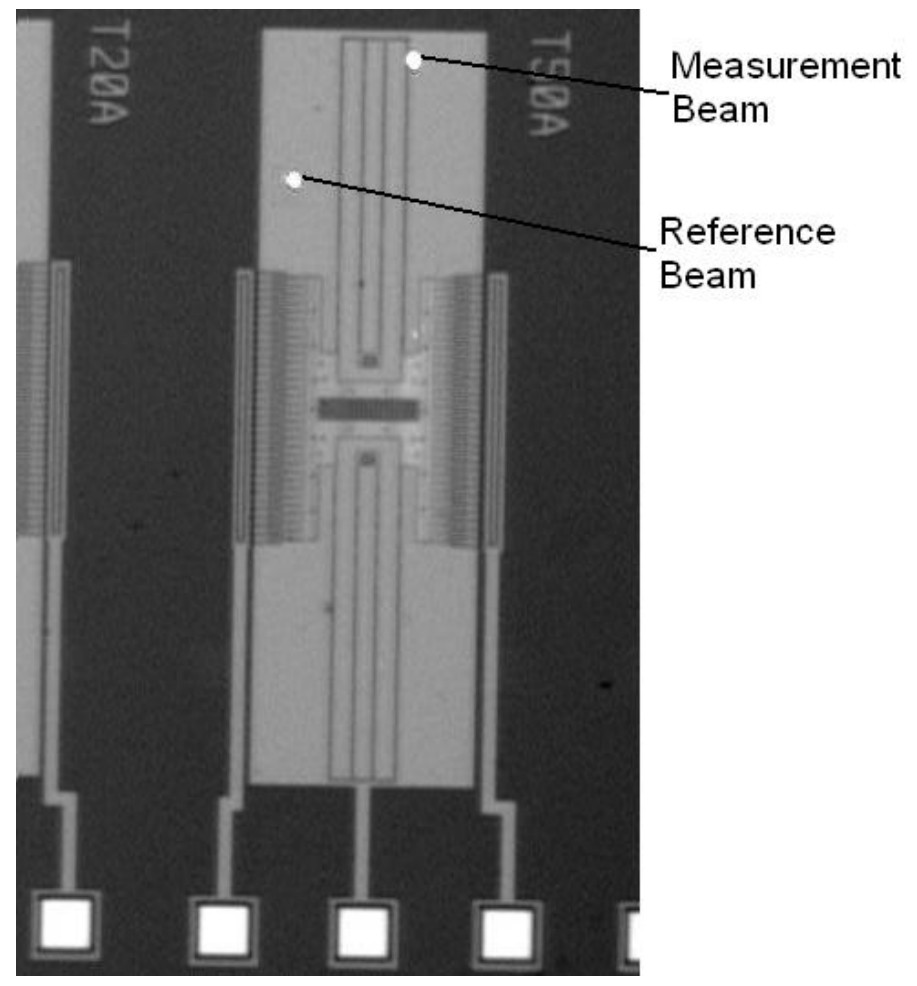

Figure 5.3: The placement of measurement and reference beams on the device

collection, were found to give similar results as expected. Fig.5.4, shows the experimental setup and the probe station used for electrical contact (for the devices which could not be bonded).

\subsection{Assumptions and recovery of optical data}

Observing the changes in the optical signal, it was assumed that it is proportional to the displacement of the system. This assumption gives a better quantitative estimate of the experimental values and the ability to compare with the simulation results. 


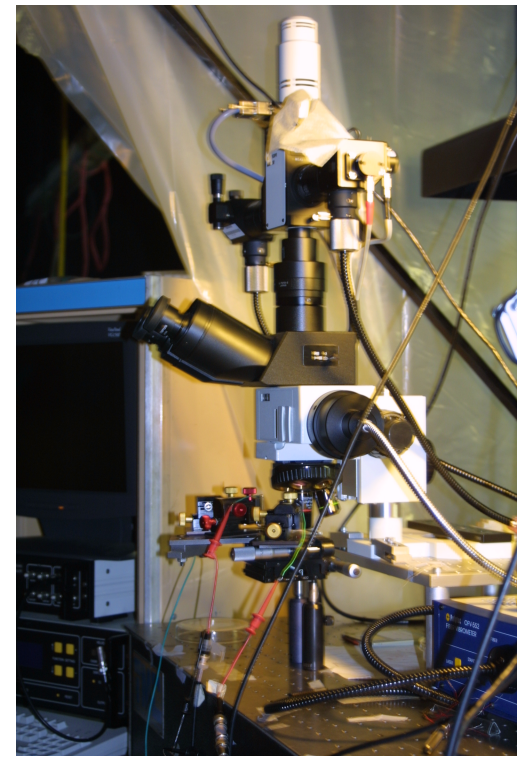

(a) Vibrometer

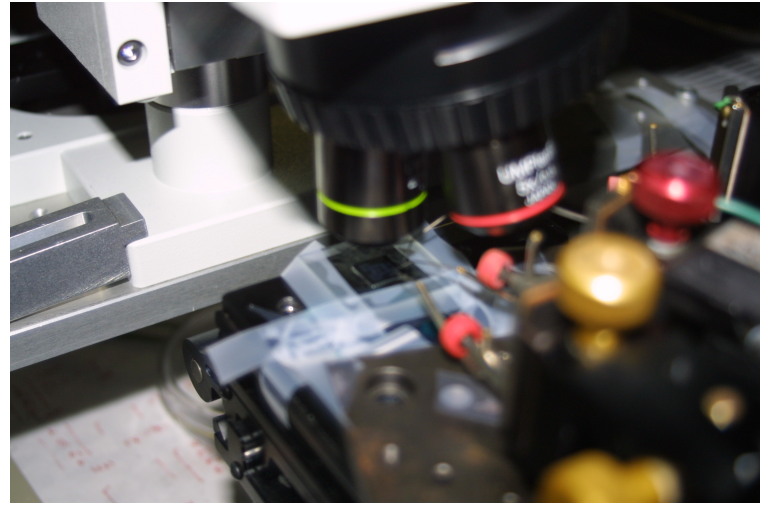

(b) Probe station

Figure 5.4: Experimental Setup

\begin{tabular}{|c|c|c|c|c|}
\hline Point of Adhesion & ANSYS difference & Optical difference & $\begin{array}{c}\text { Normalized ANSYS } \\
\text { difference }\end{array}$ & $\begin{array}{c}\text { Normalized Optical } \\
\text { difference }\end{array}$ \\
\hline 20 & $0.68 \mu \mathrm{m}$ & $8.92 \mathrm{e}-4$ & 0.658 & 0.686 \\
50 & $1.03 \mu \mathrm{m}$ & 0.0013 & 1 & 1 \\
\hline
\end{tabular}

Table 5.1: Verification

To validate the assumption made above, normalized simulated values and normalized optical values were compared. The difference in the displacement at the ends of the flexures of the devices with the point of adhesion at $20 \%$ and $50 \%$ of length of the anchor beam were taken from the simulation (at their respective resonant frequencies). The value of the optical signal taken is the mean of about 3000 points and the standard deviation of these points for various devices is given in Table 5.2. It is clear from this table that the standard deviation is about $20 \%$ of the mean value. The thus obtained optical signal difference at the ends of the flexures was taken for the same devices. These two sets of data were normalized and compared. Table 5.1 shows the 


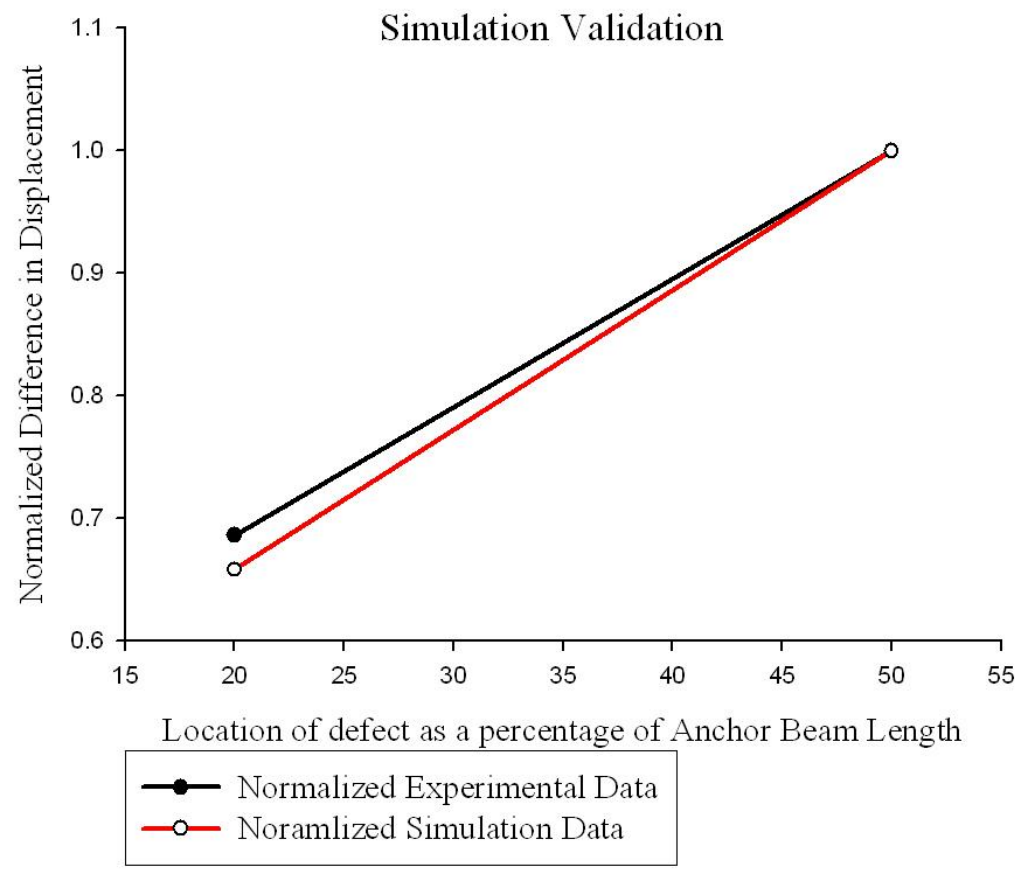

Figure 5.5: Simulation Validation

\begin{tabular}{|c|c|c|}
\hline Device & Standard Deviation & Mean \\
\hline T450 & $1.03 \mathrm{e}-5$ & $2.93 \mathrm{e}-4$ \\
T20A & $2.13 \mathrm{e}-4$ & $8.67 \mathrm{e}-4$ \\
T50A & $2.94 \mathrm{e}-4$ & $1.2 \mathrm{e}-3$ \\
T50A2 & $6.9 \mathrm{e}-4$ & $2.4 \mathrm{e}-3$ \\
T10C & $3.82 \mathrm{e}-4$ & $1.7 \mathrm{e}-3$ \\
\hline
\end{tabular}

Table 5.2: Standard Deviation

absolute values and the normalized values. Fig 5.5 shows the plot of the normalized values. 


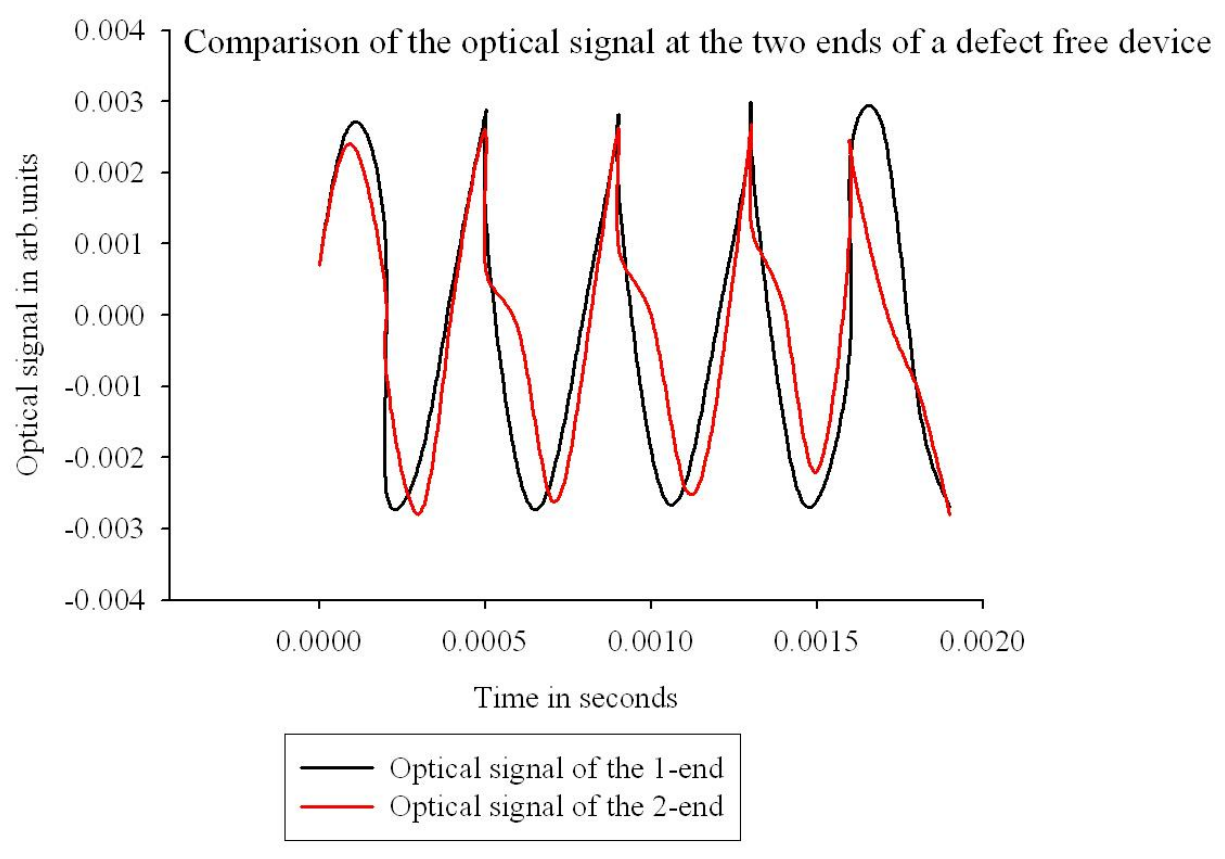

Figure 5.6: Optical signal comparison at the ends of a defect free device

From the Fig.5.6 and Fig.5.7 we can observe the optical signals when two ends of a defective and defect free device are taken. It is clear that there is a difference in the signals for the case of defective devices. Also it can be seen in Fig 5.5, the assumption that the optical signal is proportional to the displacement is reasonably valid. Therefore recovery of displacement from this optical data needs to be done. A Matlab program is written to take to the all values of local maxima of the optical signal and calculate the mean. This is done for different frequencies. This gives an estimate of the frequency response. A Gaussian curve fit of this data was done to give an estimate about the peak and the resonant frequency of the device. The values were then normalized and the peak amplitude value was changed to the expected value for that device, at that frequency. Therefore the entire frequency response was modified to give the displacement values. 


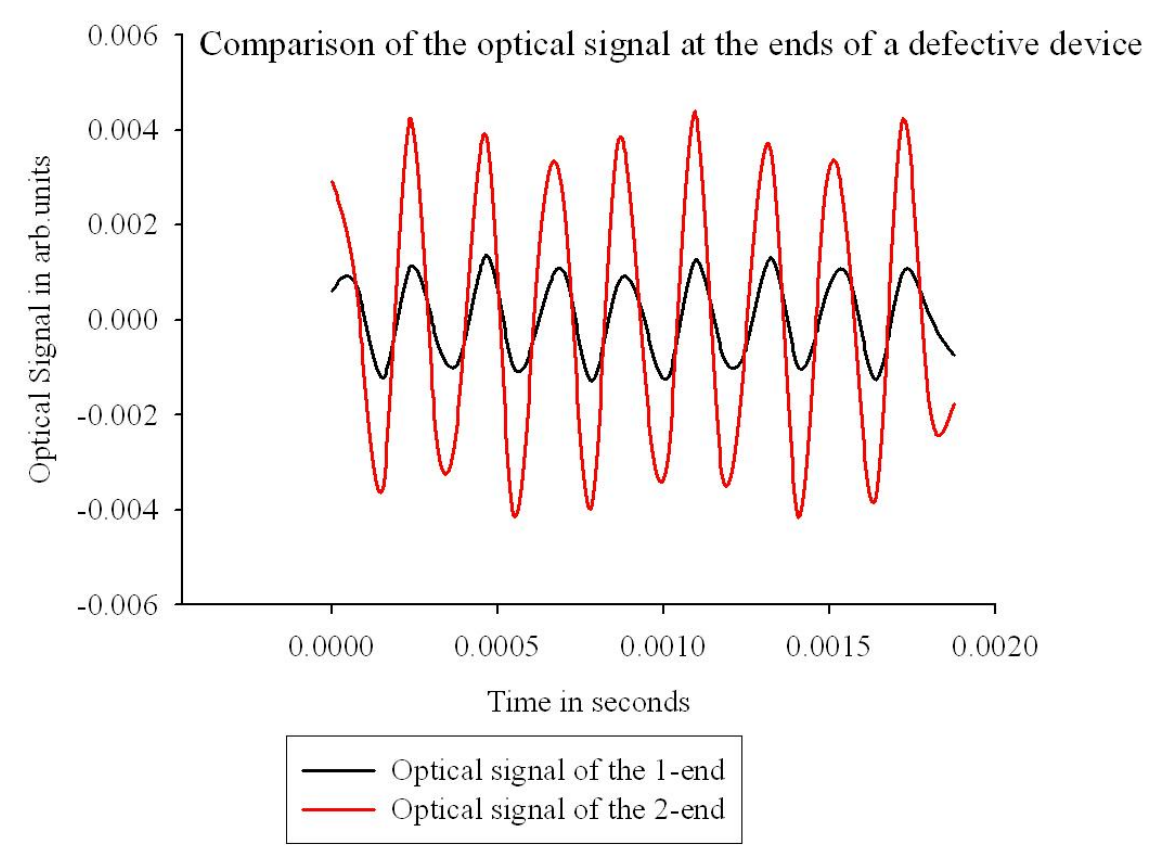

Figure 5.7: Optical signal comparison at the ends of a defective device

\subsection{Initial Results}

The Fig.5.8 shows the frequency response of the control device. This device has a flexure beam length of $450 \mu \mathrm{m}$ and beam width of $3 \mu \mathrm{m}$. The expected frequency response of the devices within the fabrication tolerances is calculated from ANSYS model. As can be observed, the experimental data lies within the envelope of the expected frequency response.

The Fig.5.9 shows the resonant frequencies of three different defective devices obtained from the experimental data. The devices taken here are, point of adhesion at $20 \%$ the length of the anchor beam, point of adhesion at $50 \%$ the length of the anchor beam and a multi defect device with point of adhesion at $50 \%$ the length of the anchor beam and a beam width of $2 \mu \mathrm{m}$ (over-etch). It is clearly observed that the resonant frequencies of these devices lie within the resonant frequency envelope of the defect free device. Hence it restates the point that the resonant frequency cannot be used to distinguish the defective devices.

The experimental frequency response and displacement of the stage of a multi defect device 


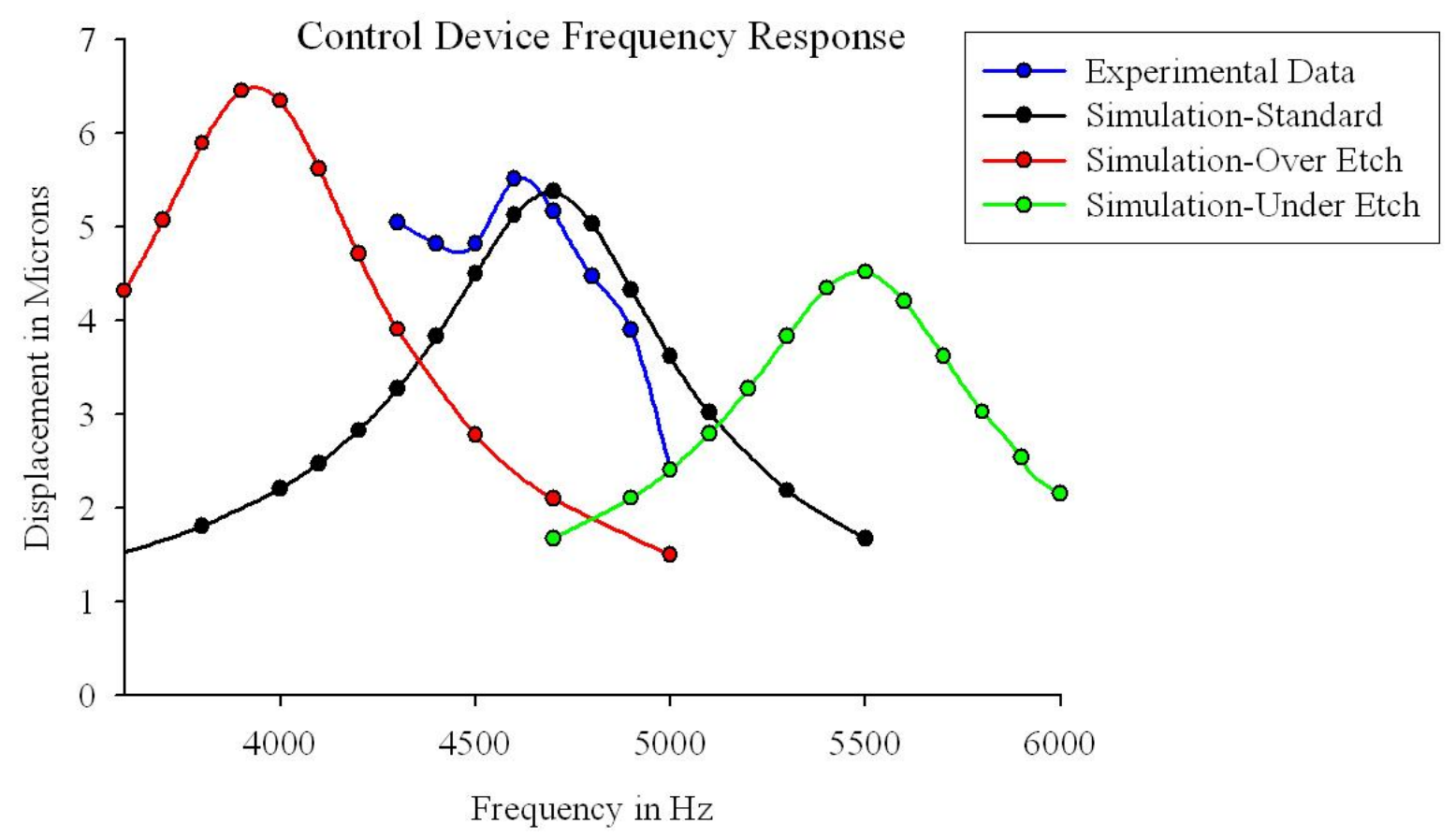

Figure 5.8: Frequency response of the T450 Device

were taken. The device considered here is a 50\% point of adhesion and $2 \mu \mathrm{m}$ anchor beam width device. The obtained frequency response was compared with that of the defect free device. Fig.5.10 shows the frequency response comparison, when the absolute values of the optical signal were taken. Fig.5.11 shows the comparison of the recovered displacement of the defect free and defective devices.

As can be clearly observed, it is not possible to distinguish between the defective and defectfree devices either by resonant frequency values or by the frequency response. This confirms with predictions made in Chapter 3. 


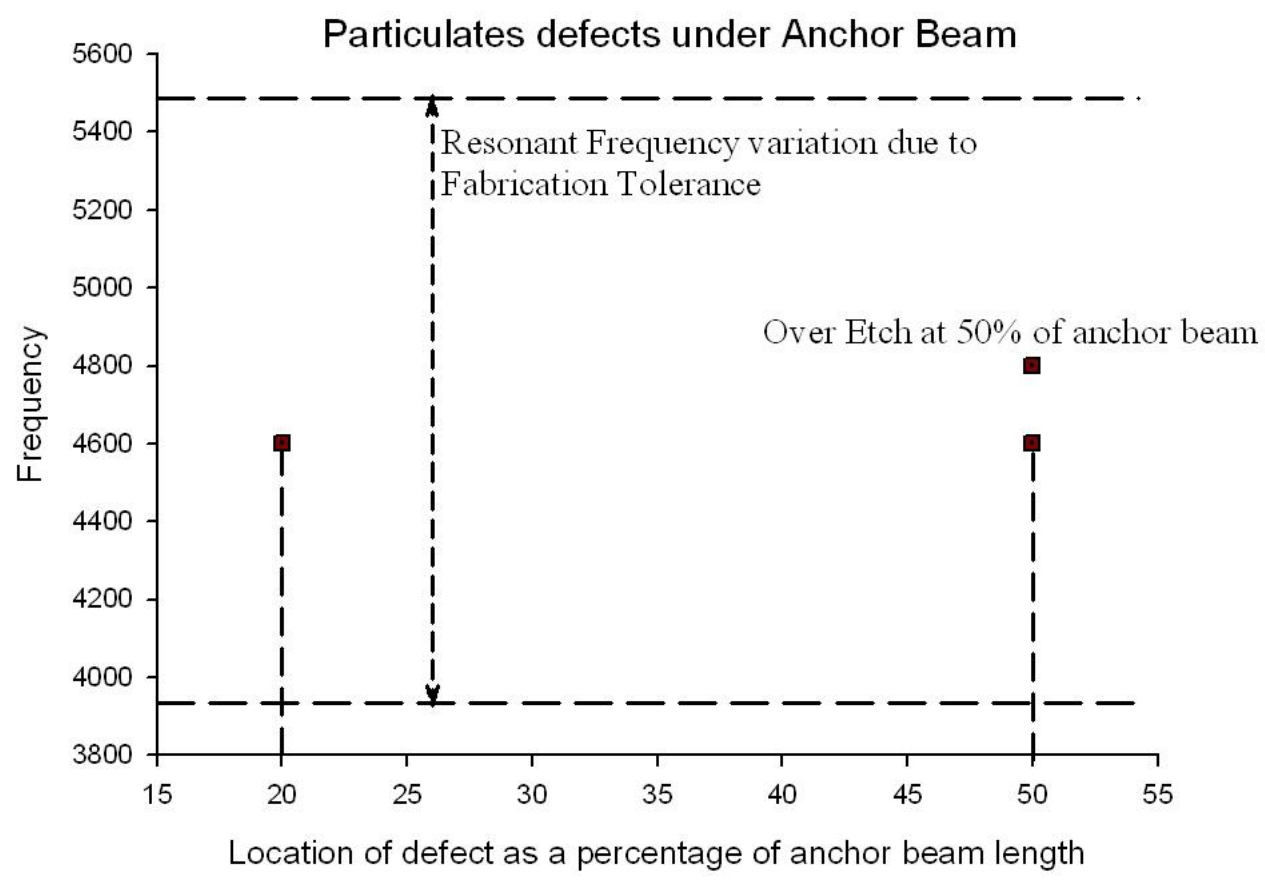

Figure 5.9: Resonant frequencies of defective devices

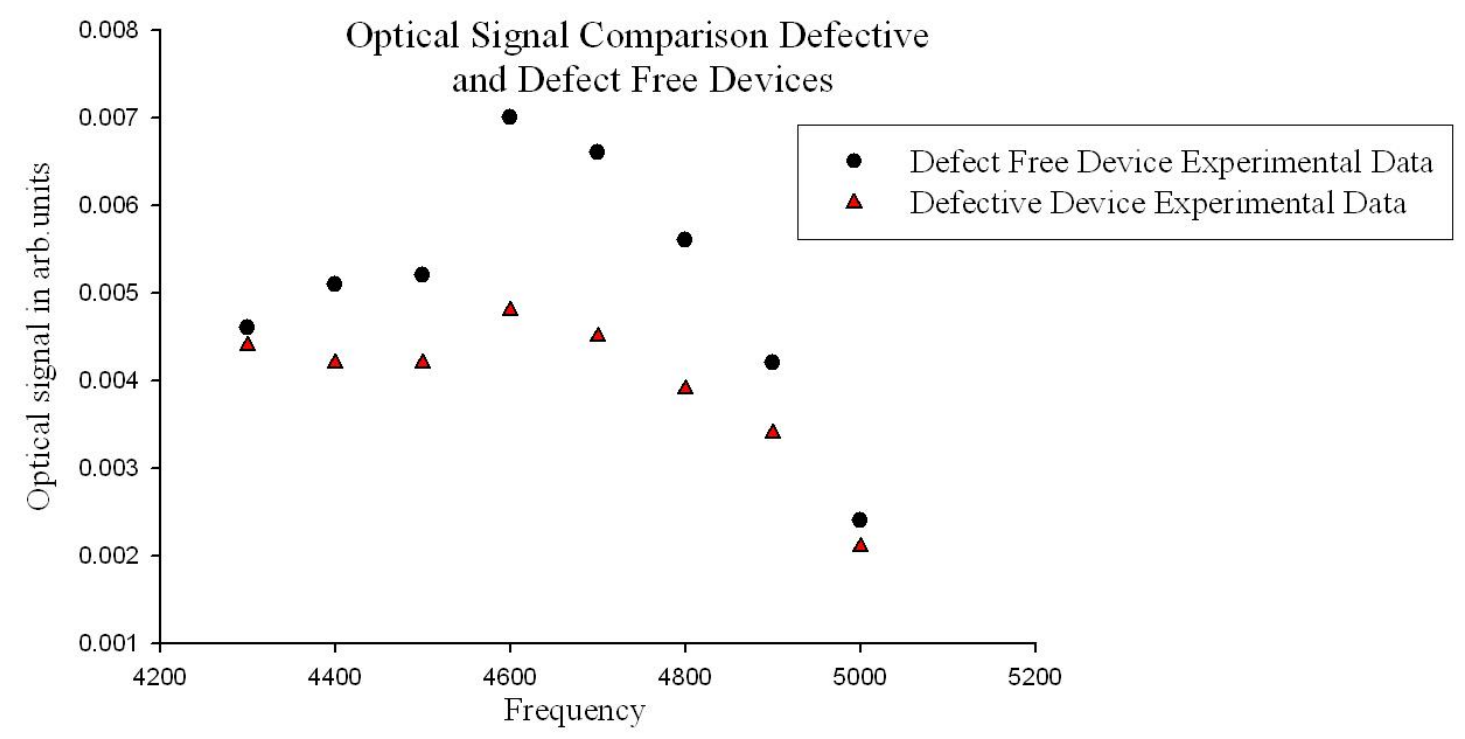

Figure 5.10: Optical signal values at the stage of a defect free and defective device 


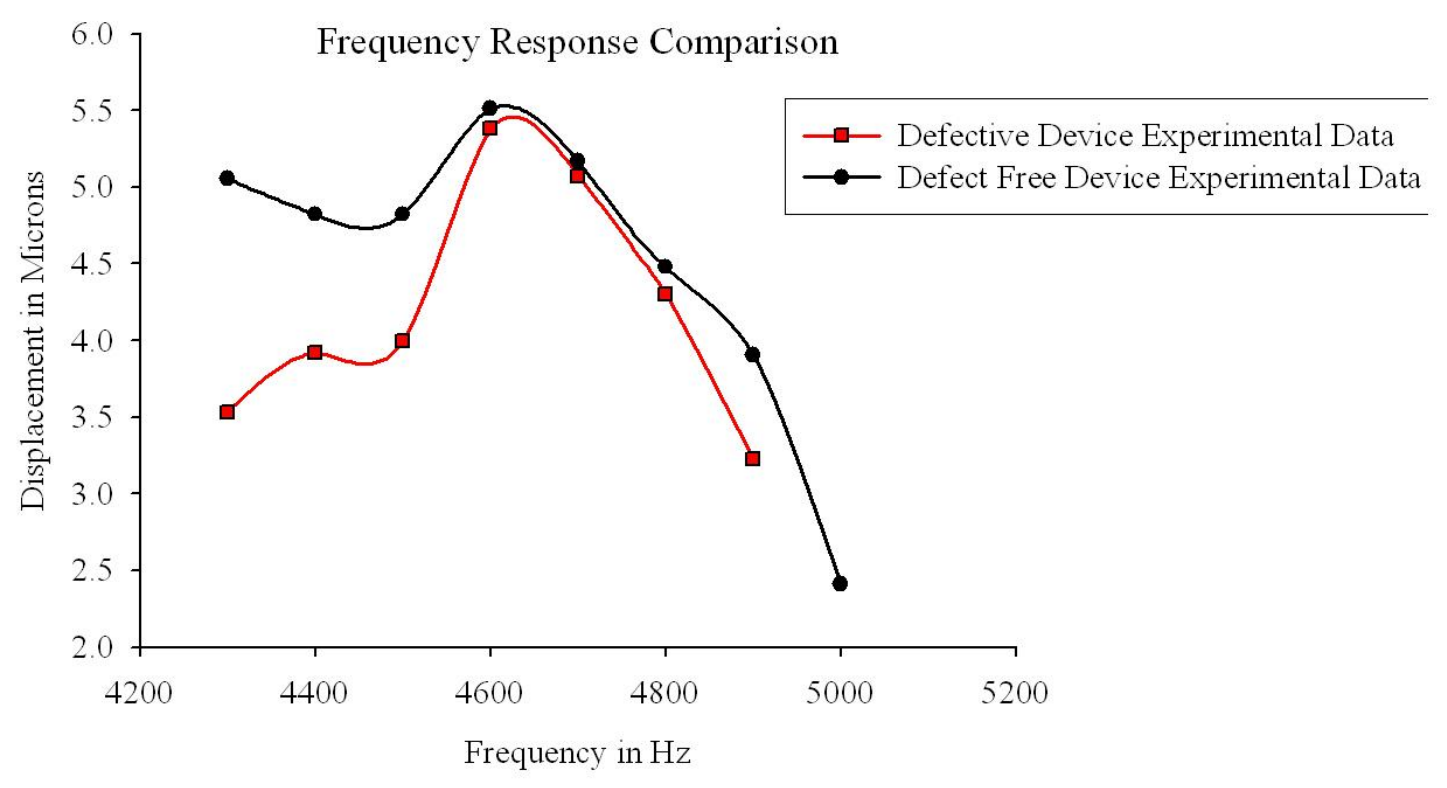

Figure 5.11: Recovered frequency response comparison of defective and defect free devices

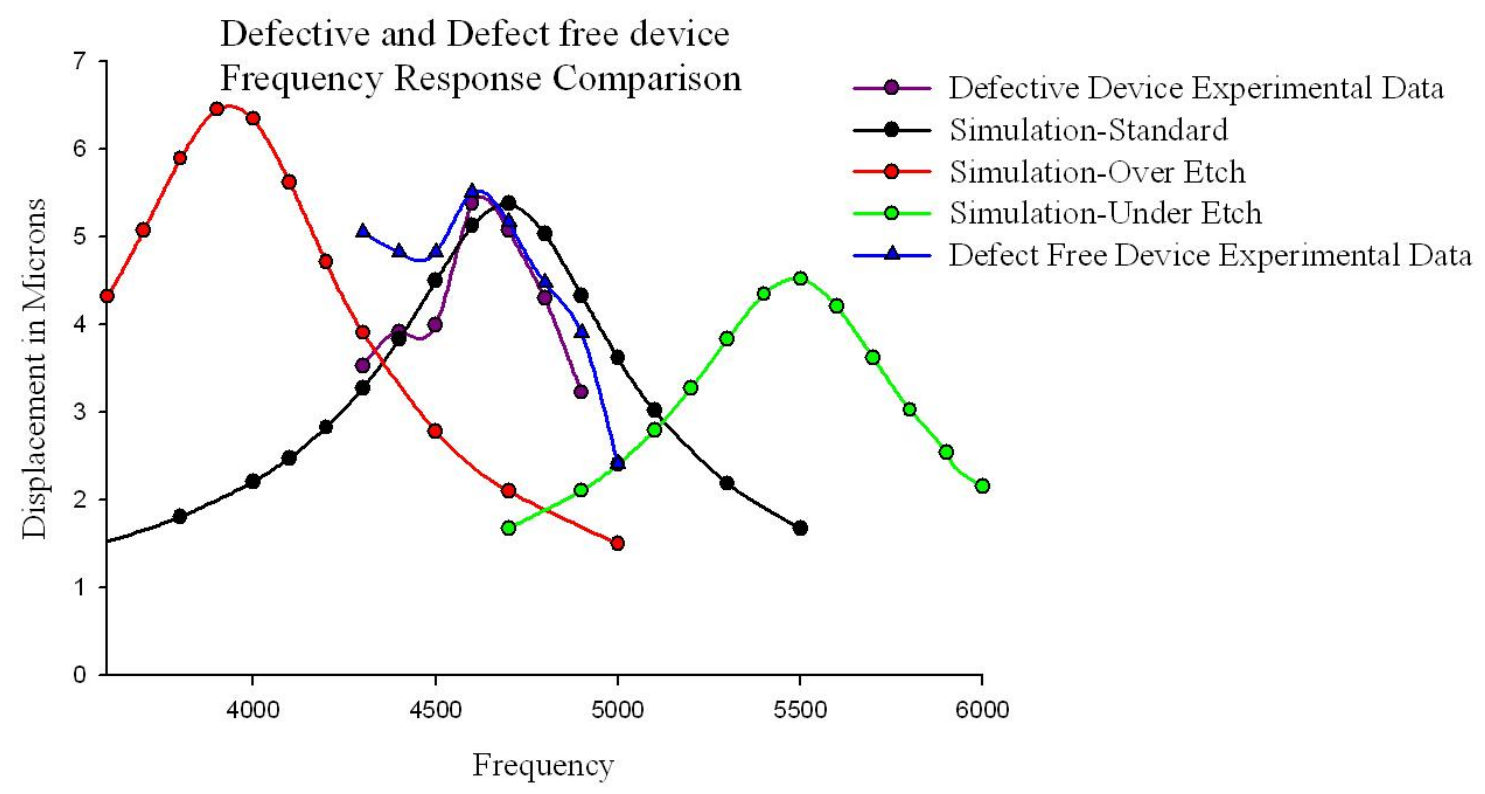

Figure 5.12: Frequency response envelope 


\subsection{Fault Detection}

In this section, it is shown that the difference in the optical signal at the ends of the flexures of the devices is sufficient for defect detection. The devices considered here are a $450 \mu \mathrm{m}$ beam length control device (defect-free device) and a $50 \%$ point of adhesion $2 \mu \mathrm{m}$ anchor beam width device(defective device). Fig.5.12, shows the frequency response of the two devices and also the expected envelope of the defect free device, due to fabrication tolerances. Fig.5.13, shows the difference in the optical signal of a defective device and a defect free device. It is clearly seen

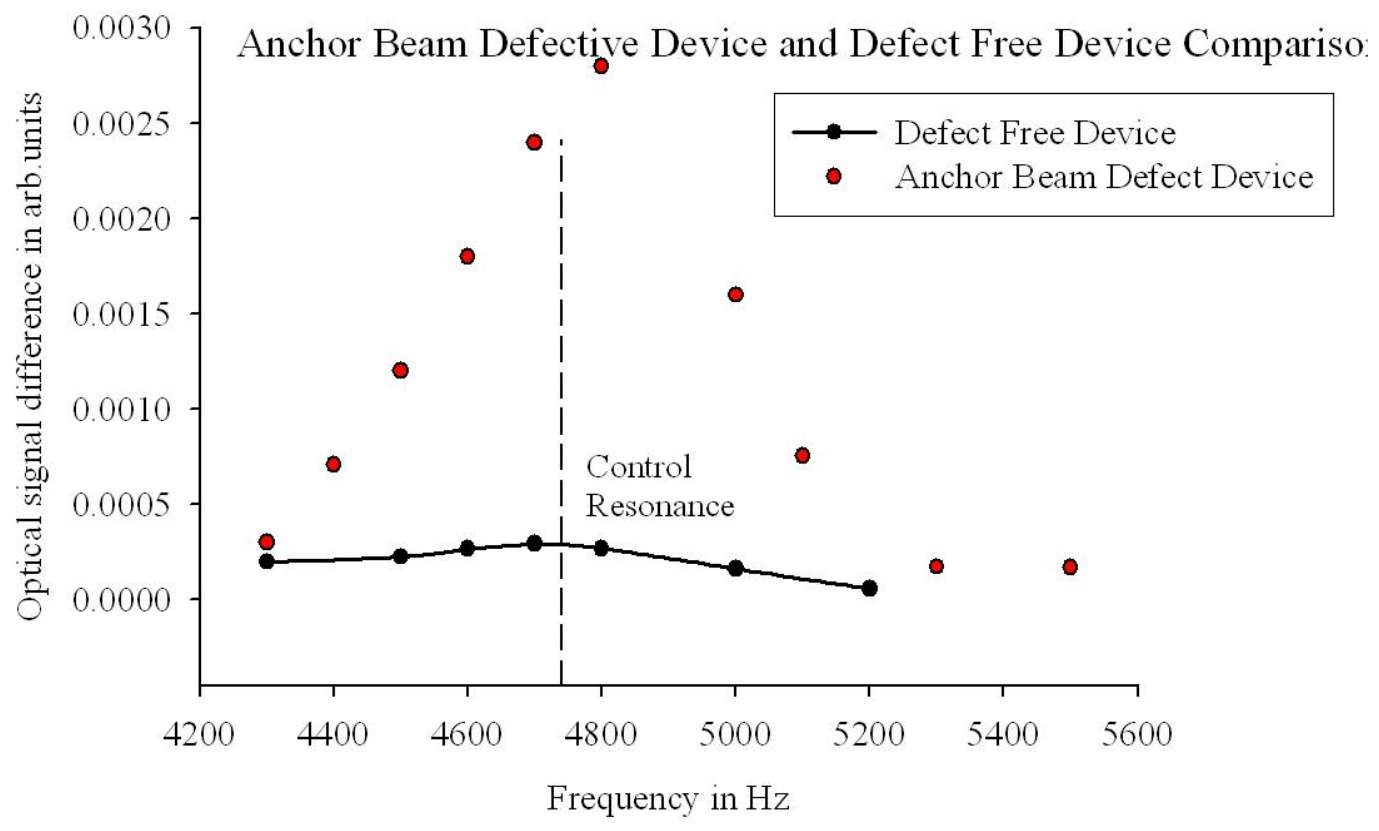

Figure 5.13: Anchor beam Multiple fault detection

that the difference optical signal of a defective device is much larger than that of a defect free device. Also, as claimed in Chapter 3, it can be observed that the signal value of the defective device at the control device's resonant frequency, is sufficient to make an optical fault detection. Fig.5.14 shows the recovered frequency response for a $20 \%$ point of adhesion device. The etch variation, that would give a device whose resonant frequency matches that of the experimental 


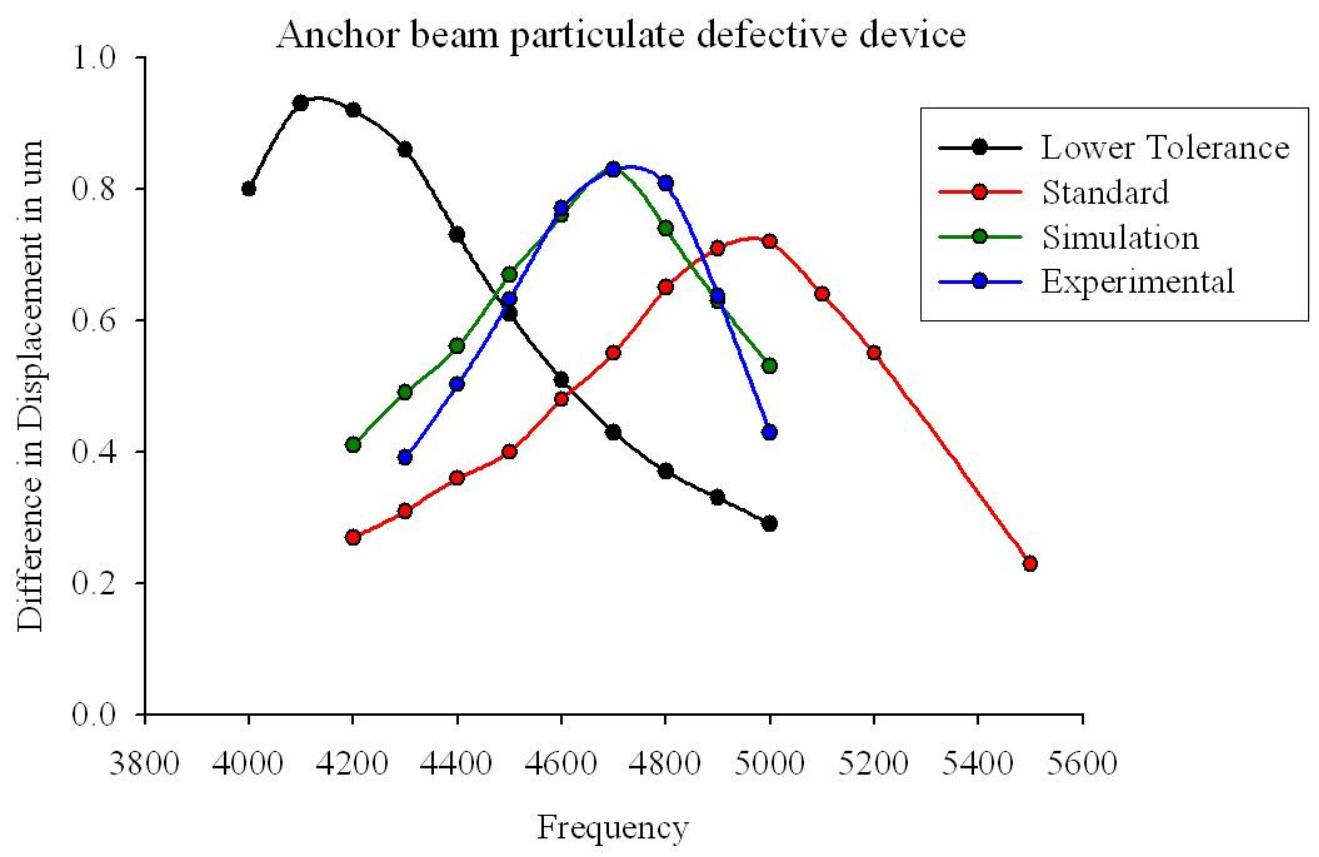

Figure 5.14: Recovered displacement of a $20 \%$ point of adhesion device

response, was computated and the simulated frequency response of such a device was calculated. As can be observed the simulation and experimental data match very closely. 


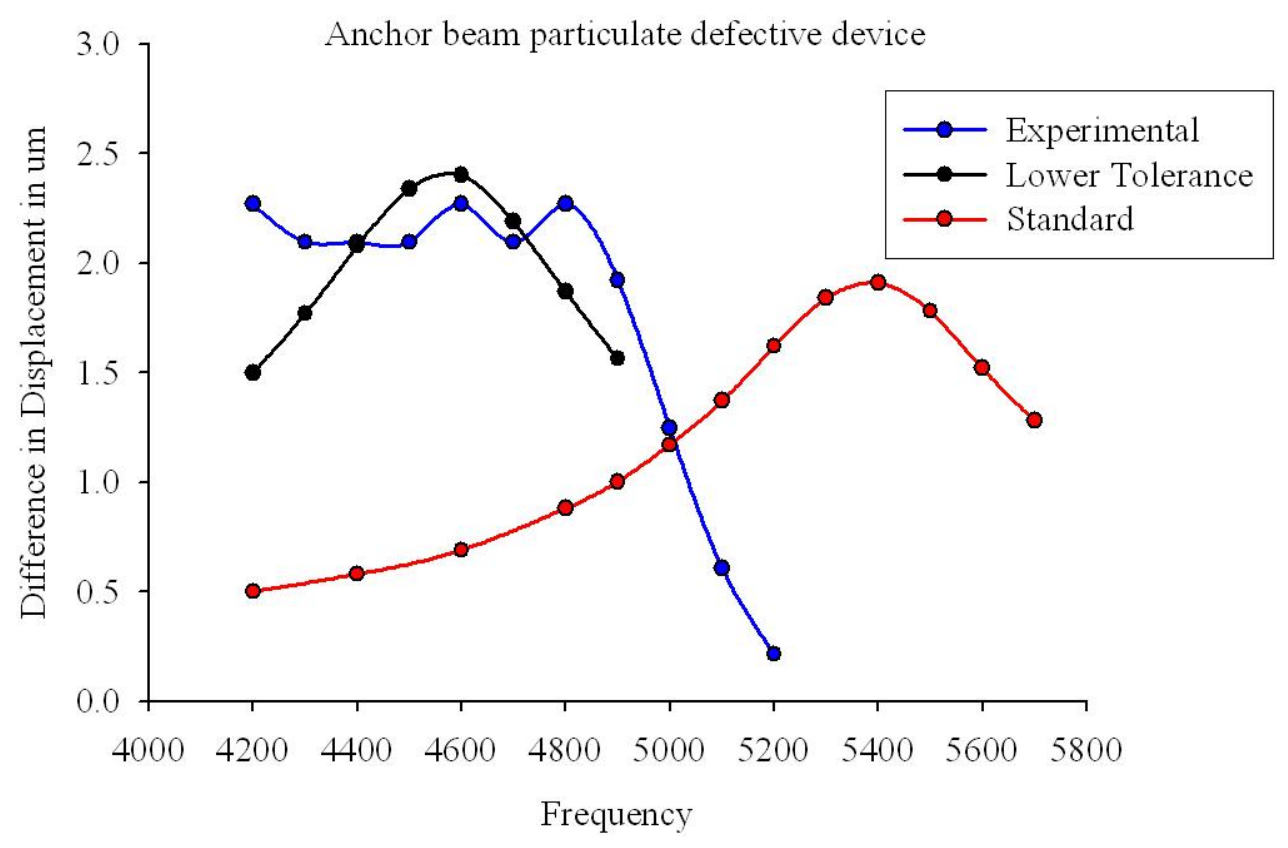

Figure 5.15: Recovered displacement of a 50\% point of adhesion device

Fig.5.15 and Fig.5.16 show similar curves for 50\% point of adhesion and 50\% point of adhesion with over-etched anchor beam device. Simulations closest to the experimental data could not be estimated in this case, because the etch variations that might lead to such a response are difficult to simulate. As can be observed the experimental data lies within the expected frequency envelope. The experimental data has a sharper curve in Fig.5.16 because variations in the environmental conditions cause variations in damping and thus cause changes in the frequency response. Fig.5.17, shows the optical signal comparison at different frequencies of four different devices. All of these devices have their point of adhesion on the anchor beam. As expected, as the point of adhesion moves away from the anchor, the difference signal amplitude increases. This occurs because the asymmetry between the devices becomes more pronounced as the points of adhesion moves towards points of greater amplitude. 


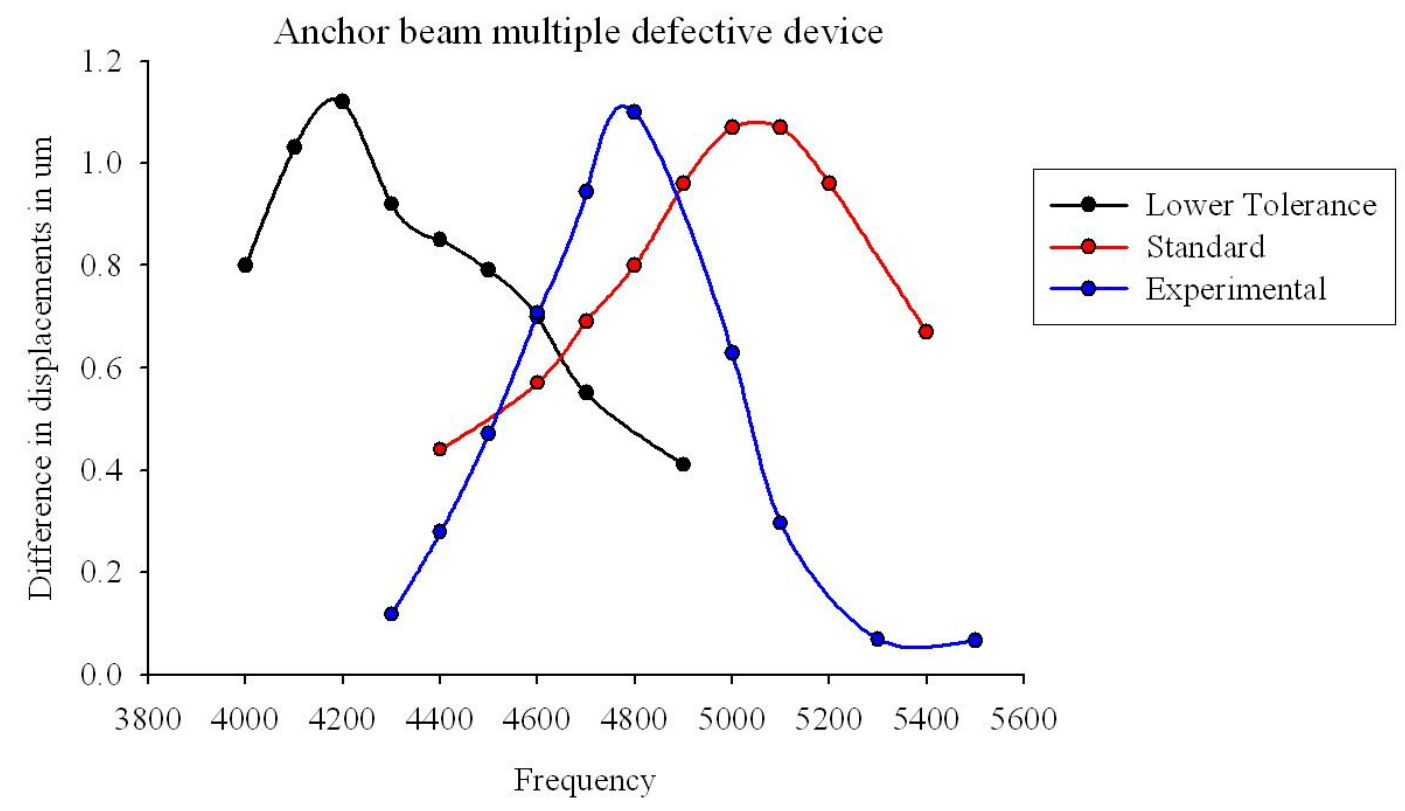

Figure 5.16: Recovered displacement of an over-etched 50\% point of adhesion device

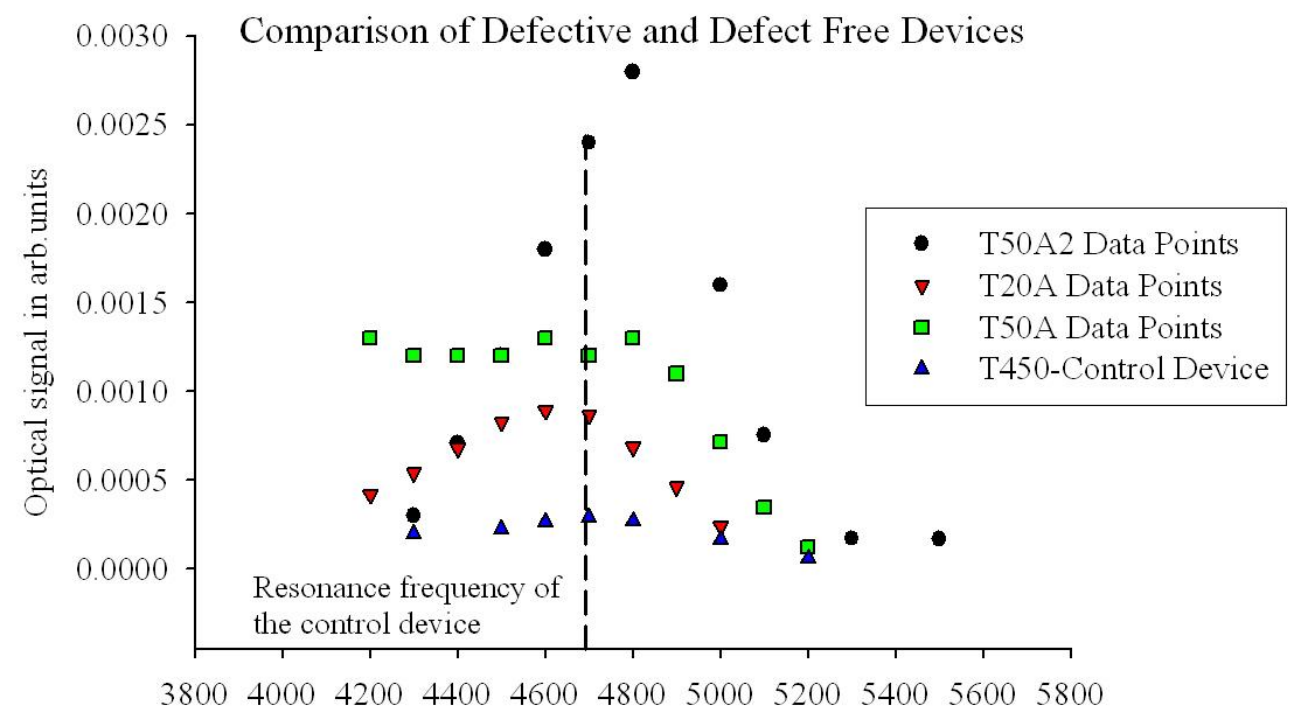

Frequency

Figure 5.17: Optical detection of Faults:Anchor Beam 
In the following figures the fault detection of a corner beam is shown. The devices considered here are devices having points of adhesion at $10 \%$ of the corner beam length and $10 \%$ point of adhesion with over-etching of a single beam. Fig. 5.18 shows the optical signal comparison with

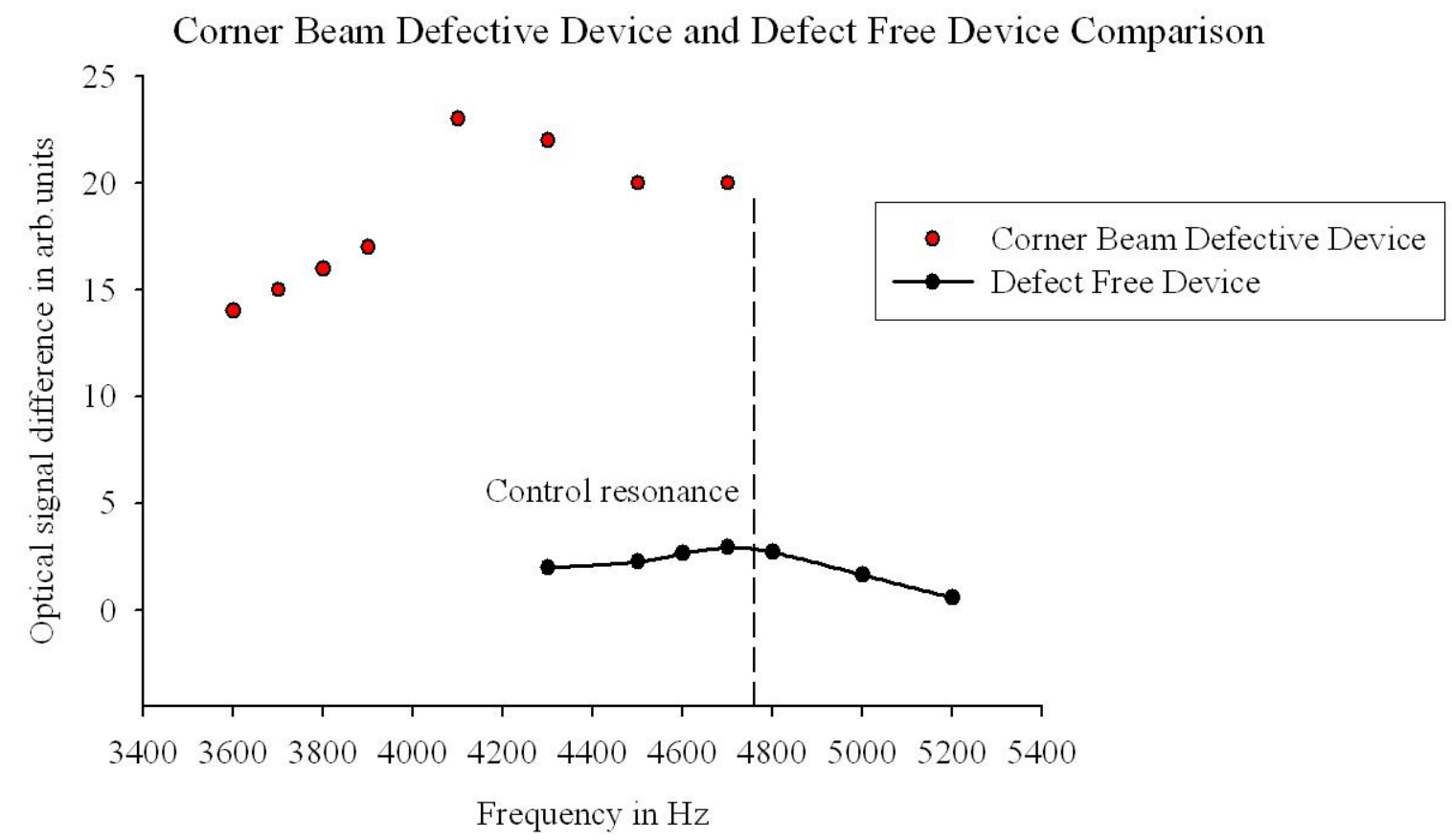

Figure 5.18: Corner beam multi fault detection

a defect free device. It can be seen that, though the resonant frequency of the defective device is closer to the lower tolerance resonant frequency of the defect free device, its defective nature can be detected by the optical signal at the resonant frequency of the defect free device. Fig.5.19 shows the recovered displacement and envelope created by the fabrication tolerances. The device behavior can be observed to be closest to the lower tolerance frequency response.

Fig. 5.20 shows the $10 \%$ corner beam device with over-etching and no etch variation. As expected the value of the optical signal for the over-etch case is much higher than that of the no-etch variation case. Fig.5.21, shows the comparison of the simulation and the experimental data. Here the difference in the response of the two curves can be attributed to the non-ideal environmental conditions and also to the errors in the experiments. 


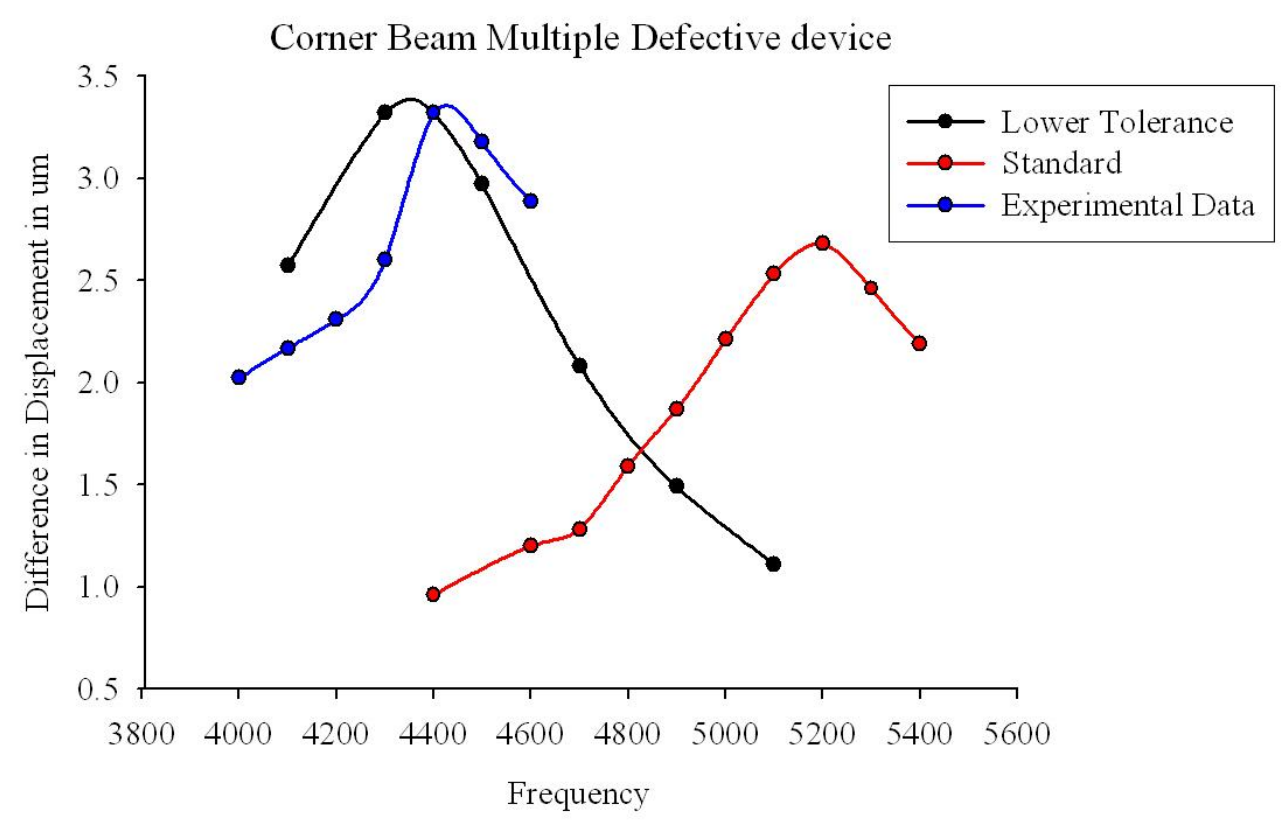

Figure 5.19: Recovered displacement of an over-etched 10\% point of adhesion device:Corner Beam

Comparison of Defective Corner Beam and Defect Free Devices

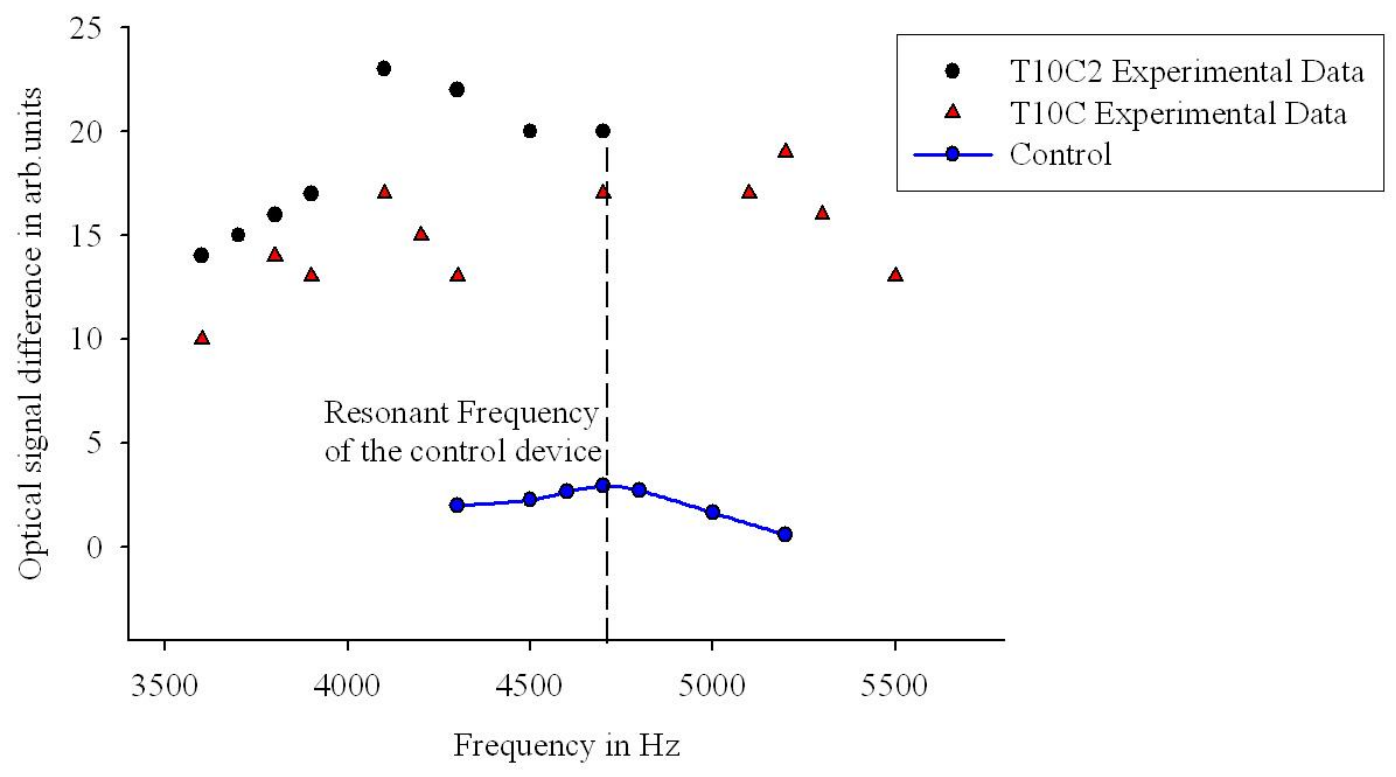

Figure 5.20: Optical detection of Faults:Corner Beam 


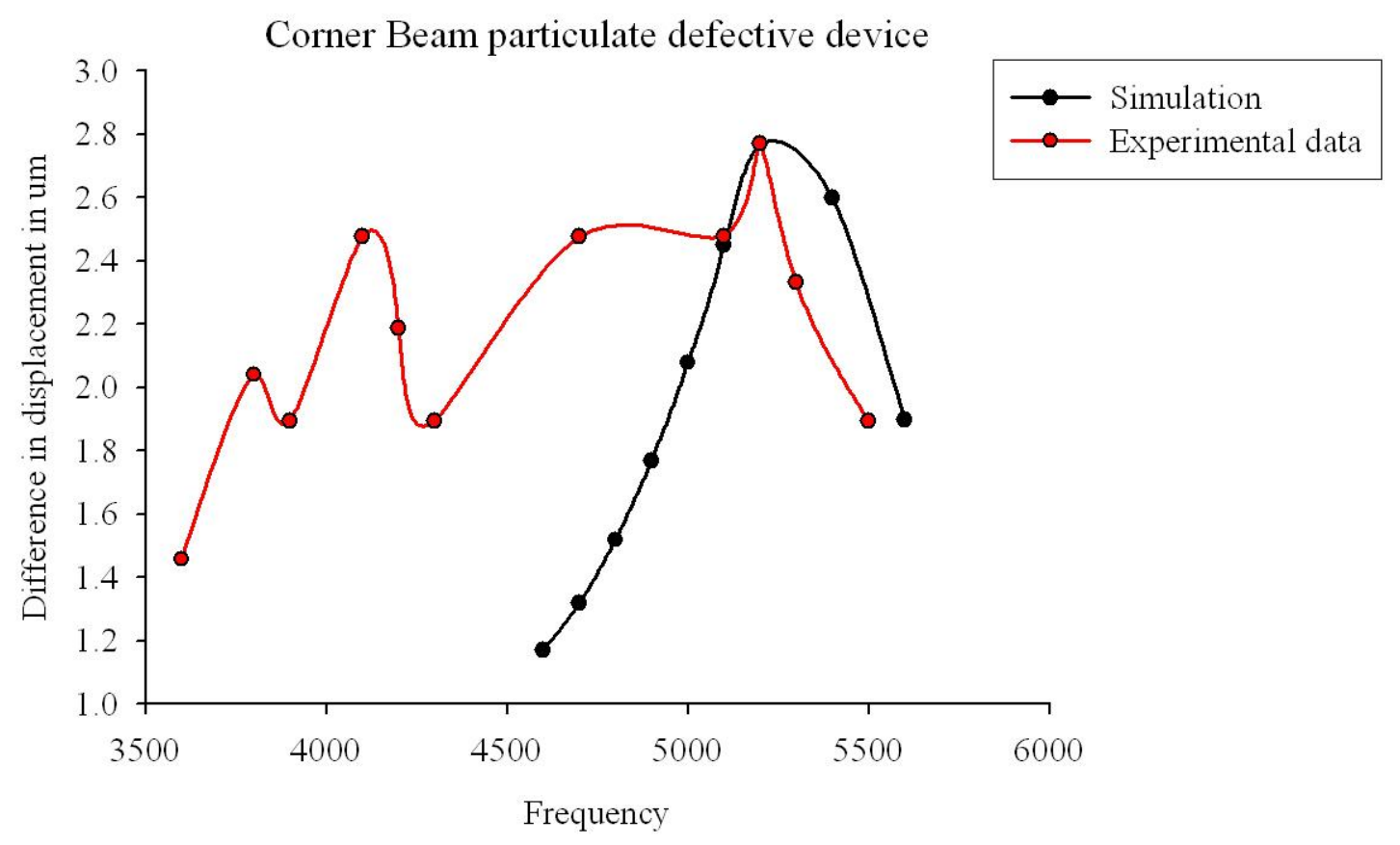

Figure 5.21: Recovered displacement of a 10\% point of adhesion device:Corner Beam 


\section{Chapter 6}

\section{Conclusions and Future Work}

The increasing use of MEMS in safety critical systems has made the reliability of these devices a very important issue. A decoupled measurement system enables us to conduct lifetime in situ monitoring of these devices, without interrupting their normal operation. It is shown here that multiple faults on the devices which cause behavior masking, can be detected by optical probing, thus improving the reliability of the systems and enabling life-time control.

Simulations in Chapter 3 indicate that the solid model that is developed, can be used for the determination of the stress and defect analysis of the system effectively. It is also shown that general parameters such as resonant frequency cannot be used to distinguish between defective and defect free devices. It is also illustrated that these devices can be distinguished by measuring the displacements at the ends of the flexures.

In Chapter 5 it is experimentally shown that the multi-defective and defect free devices can be identified by optically probing the ends of the flexures. The reflected optical signal, is shown to be proportional to the displacement of the point at which the signal is collected. It is shown that the difference in the optical signals of a defective device is about 10 times the difference in the optical signal of defect free device. Hence such a difference gives us a clear indication of the defective and defect free devices. These differences are consistent with those predicted in chapter 3. 


\subsection{Future Work}

In this thesis, multiple defects causing asymmetry have been discussed. Faults occurring on both sides of the flexure need to be detected using a stress analysis, as they do not create an asymmetry. Also, the results obtained here, need to be verified using through wafer optical system for integration with the optical package. Lenses giving multiple probe beams, for measurement at both ends of the flexures need to be designed. 


\section{Appendix A}

\section{ANSYS Codes}

Code for the preprocessing of ANSYS model This shows the development of the solid model and the material input given. Rest of the model can be done by graphic selection.

/prep7 blc4,-530e-6,45e-6,450e-6,2e-6 blc4,-530e-6,15e-6,2e-6,30e-6 blc4,-530e-6,13e-6,450e-6,2e-6 blc4,-530e-6,-13e-6,2e-6,26e-6 blc4,-80e-6,-15e-6,20e-6,30e-6 blc4,-80e-6,45e-6,80e-6,25e-6 blc4,-188e-6,70e-6,188e-6,20e-6 blc4,-40e-6,0,40e-6,45e-6

blc4,-15e-6,69e-6,15e-6,2e-6 blc4,-15e-6,65e-6,15e-6,2e-6 blc4,-15e-6,61e-6,15e-6,2e-6 blc4,-15e-6,57e-6,15e-6,2e-6 blc4,-15e-6,53e-6,15e-6,2e-6 blc4,-15e-6,49e-6,15e-6,2e-6 blc4,-15e-6,45e-6,15e-6,2e-6 blc4,-15e-6,41e-6,15e-6,2e-6 blc4,-15e-6,37e-6,15e-6,2e-6 blc4,-15e-6,33e-6,15e-6,2e-6 blc4,-15e-6,29e-6,15e-6,2e-6 blc $4,-15 e-6,25 e-6,15 e-6,2 e-6$ blc4,-15e-6,21e-6,15e-6,2e-6 blc4,-15e-6,17e-6,15e-6,2e-6 blc4,-15e-6,13e-6,15e-6,2e-6 blc4,-15e-6,9e-6,15e-6,2e-6 blc4,-15e-6,5e-6,15e-6,2e-6 blc4,-15e-6,1e-6,15e-6,2e-6

blc4,-188e-6,90e-6,4e-6,50e-6 blc4,-176e-6,90e-6,4e-6,50e-6 
blc4,-164e-6,90e-6,4e-6,50e-6 blc4,-152e-6,90e-6,4e-6,50e-6 blc4,-140e-6,90e-6,4e-6,50e-6 blc4,-128e-6,90e-6,4e-6,50e-6 blc4,-116e-6,90e-6,4e-6,50e-6 blc4,-104e-6,90e-6,4e-6,50e-6 blc4,-92e-6,90e-6,4e-6,50e-6 blc4,-80e-6,90e-6,4e-6,50e-6 blc4,-68e-6,90e-6,4e-6,50e-6 blc4,-56e-6,90e-6,4e-6,50e-6 blc4,-44e-6,90e-6,4e-6,50e-6 blc4,-32e-6,90e-6,4e-6,50e-6 blc4,-20e-6,90e-6,4e-6,50e-6 blc4,-8e-6,90e-6,4e-6,50e-6

blc4,80e-6,45e-6,450e-6,2e-6 blc4,528e-6,15e-6,2e-6,30e-6 blc4,80e-6,13e-6,450e-6,2e-6 blc4,528e-6,-13e-6,2e-6,26e-6 blc4,60e-6,-15e-6,20e-6,30e-6 blc4,0,45e-6,80e-6,25e-6 blc4,0,70e-6,188e-6,20e-6 blc4,0,0,40e-6,45e-6 blc4,0,69e-6,15e-6,2e-6 blc4,0,65e-6,15e-6,2e-6 blc4,0,61e-6,15e-6,2e-6 blc4,0,57e-6,15e-6,2e-6 blc4,0,53e-6,15e-6,2e-6 blc4,0,49e-6,15e-6,2e-6 blc4,0,45e-6,15e-6,2e-6 blc4,0,41e-6,15e-6,2e-6 blc4,0,37e-6,15e-6,2e-6 blc4,0,33e-6,15e-6,2e-6 blc4,0,29e-6,15e-6,2e-6 blc4,0,25e-6,15e-6,2e-6 blc4,0,21e-6,15e-6,2e-6 blc4,0,17e-6,15e-6,2e-6 blc4,0,13e-6,15e-6,2e-6 blc4,0,9e-6,15e-6,2e-6 blc4,0,5e-6,15e-6,2e-6 blc4,0,1e-6,15e-6,2e-6 blc4,184e-6,90e-6,4e-6,50e-6 blc4,172e-6,90e-6,4e-6,50e-6 blc4,160e-6,90e-6,4e-6,50e-6 blc4,148e-6,90e-6,4e-6,50e-6 
blc4,136e-6,90e-6,4e-6,50e-6 blc4,124e-6,90e-6,4e-6,50e-6 blc4,112e-6,90e-6,4e-6,50e-6 blc4,100e-6,90e-6,4e-6,50e-6 blc4,88e-6,90e-6,4e-6,50e-6 blc4,76e-6,90e-6,4e-6,50e-6 blc4,64e-6,90e-6,4e-6,50e-6 blc4,52e-6,90e-6,4e-6,50e-6 blc4,40e-6,90e-6,4e-6,50e-6 blc4,28e-6,90e-6,4e-6,50e-6 blc4,16e-6,90e-6,4e-6,50e-6 blc4,4e-6,90e-6,4e-6,50e-6

blc4,-530e-6,-47e-6,450e-6,2e-6 blc4,-530e-6,-45e-6,2e-6,30e-6 blc4,-530e-6,-15e-6,450e-6,2e-6 blc4,-80e-6,-70e-6,80e-6,25e-6 blc4,-188e-6,-90e-6,188e-6,20e-6 blc4,-40e-6,-45e-6,40e-6,45e-6 blc4,-15e-6,-71e-6,15e-6,2e-6 blc4,-15e-6,-67e-6,15e-6,2e-6 blc4,-15e-6,-63e-6,15e-6,2e-6 blc4,-15e-6,-59e-6,15e-6,2e-6 blc4,-15e-6,-55e-6,15e-6,2e-6 blc4,-15e-6,-51e-6,15e-6,2e-6 blc4,-15e-6,-47e-6,15e-6,2e-6 blc4,-15e-6,-43e-6,15e-6,2e-6 blc4,-15e-6,-39e-6,15e-6,2e-6 blc4,-15e-6,-35e-6,15e-6,2e-6 blc4,-15e-6,-31e-6,15e-6,2e-6 blc4,-15e-6,-27e-6,15e-6,2e-6 blc4,-15e-6,-23e-6,15e-6,2e-6 blc4,-15e-6,-19e-6,15e-6,2e-6 blc4,-15e-6,-15e-6,15e-6,2e-6 blc4,-15e-6,-11e-6,15e-6,2e-6 blc4,-15e-6,-7e-6,15e-6,2e-6 blc4,-15e-6,-3e-6,15e-6,2e-6

blc4,-188e-6,-140e-6,4e-6,50e-6 blc4,-176e-6,-140e-6,4e-6,50e-6 blc4,-164e-6,-140e-6,4e-6,50e-6 blc4,-152e-6,-140e-6,4e-6,50e-6 blc4,-140e-6,-140e-6,4e-6,50e-6 blc4,-128e-6,-140e-6,4e-6,50e-6 blc4,-116e-6,-140e-6,4e-6,50e-6 blc4,-104e-6,-140e-6,4e-6,50e-6 blc4,-92e-6,-140e-6,4e-6,50e-6 blc4,-80e-6,-140e-6,4e-6,50e-6 blc4,-68e-6,-140e-6,4e-6,50e-6 blc4,-56e-6,-140e-6,4e-6,50e-6 blc4,-44e-6,-140e-6,4e-6,50e-6 blc4,-32e-6,-140e-6,4e-6,50e-6 
blc4,-20e-6,-140e-6,4e-6,50e-6 blc4,-8e-6,-140e-6,4e-6,50e-6

blc4,80e-6,-47e-6,450e-6,2e-6 blc4,528e-6,-45e-6,2e-6,30e-6 blc4,80e-6,-15e-6,450e-6,2e-6

blc4,0,-70e-6,80e-6,25e-6 blc4,0,-90e-6,188e-6,20e-6 blc4,0,-45e-6,40e-6,45e-6

blc4,0,-71e-6,15e-6,2e-6 blc4,0,-67e-6,15e-6,2e-6 blc4,0,-63e-6,15e-6,2e-6 blc4,0,-59e-6,15e-6,2e-6 blc4,0,-55e-6,15e-6,2e-6 blc4,0,-51e-6,15e-6,2e-6 blc4,0,-47e-6,15e-6,2e-6 blc4,0,-43e-6,15e-6,2e-6 blc4,0,-39e-6,15e-6,2e-6 blc4,0,-35e-6,15e-6,2e-6 blc4,0,-31e-6,15e-6,2e-6 blc4,0,-27e-6,15e-6,2e-6 blc4,0,-23e-6,15e-6,2e-6 blc4,0,-19e-6,15e-6,2e-6 blc4,0,-15e-6,15e-6,2e-6 blc4,0,-11e-6,15e-6,2e-6 blc4,0,-7e-6,15e-6,2e-6 blc4,0,-3e-6,15e-6,2e-6

blc4,184e-6,-140e-6,4e-6,50e-6 blc4,172e-6,-140e-6,4e-6,50e-6 blc4,160e-6,-140e-6,4e-6,50e-6 blc4,148e-6,-140e-6,4e-6,50e-6 blc4,136e-6,-140e-6,4e-6,50e-6 blc4,124e-6,-140e-6,4e-6,50e-6 blc4,112e-6,-140e-6,4e-6,50e-6 blc4,100e-6,-140e-6,4e-6,50e-6 blc4,88e-6,-140e-6,4e-6,50e-6 blc4,76e-6,-140e-6,4e-6,50e-6 blc4,64e-6,-140e-6,4e-6,50e-6 blc4,52e-6,-140e-6,4e-6,50e-6 blc4,40e-6,-140e-6,4e-6,50e-6 blc4,28e-6,-140e-6,4e-6,50e-6 blc4,16e-6,-140e-6,4e-6,50e-6 blc4,4e-6,-140e-6,4e-6,50e-6 ET,1,PLANE82

KEYOPT,1,3,3 
KEYOPT,1,5,0

KEYOPT,1,6,0

R, 1,2e-6,

$\mathrm{R}, 2,4 \mathrm{e}-6$,

MPTEMP,,,,,,,

MPTEMP, 1,0

MPDATA,EX,1,,160E9

MPDATA,PRXY,1,0.22

MPTEMP,,,,,,,

MPTEMP, 1,0

MPDATA,DENS,1,,2330 


\section{Appendix B}

\section{MATLAB Codes}

\%damping.m used for the Calculation of Quality factor, damping ratio(dr) and c clear all;

$\mathrm{mu}=1.83 \mathrm{e}-5$; \%air visocity @ 18c

rho=1.22; \%density of air @ 18c

dens $=2330$;

$d=2 e-6$;

$\mathrm{dc}=2 \mathrm{e}-6$;

$\mathrm{t}=2 \mathrm{e}-6$;

$\mathrm{Ac}=20 \mathrm{e}-6 * \mathrm{t} ; \%$ area of comb overlap

$f=2591.7$;

$\mathrm{w}=2 * \mathrm{pi}^{*} \mathrm{f}$

$w b=2 e-6$;

$\mathrm{wt}=2 \mathrm{e}-6$;

$\mathrm{lb}=450 \mathrm{e}-6$;

lt $1=30 \mathrm{e}-6$;

lt2=26e-6;

$\mathrm{Ap}=((40 * 45 * 4)+(188 * 20 * 4)+(80 * 25 * 4)+(4 * 50 * 64)-(30 * 2 * 36)) * 1 \mathrm{e}-12$

$A t=(l t 1 * w t * 4)+(l t 2 * w t * 2) ; A b=(l b * w b * 7)+(l b 1 * w b) ;$ 
$\operatorname{delta}=\operatorname{sqrt}\left((2 * \mathrm{mu}) /\left(\mathrm{rho}^{*} \mathrm{w}\right)\right) ; \mathrm{Aq}=\mathrm{Ap}+(0.5 *(\mathrm{At}+\mathrm{Ab}))$;

$\mathrm{mb}=\mathrm{Ab} * \mathrm{t}^{*} \mathrm{dens} ; \%$ mass of the beam

$\mathrm{mt}=\mathrm{At} * \mathrm{t}^{*} \mathrm{dens} ; \%$ mass of the truss

$\mathrm{ms}=\mathrm{Ap} * \mathrm{t} *$ dens; $\%$ mass of the stage

$\mathrm{m}=\mathrm{ms}+\left(0.25^{*} \mathrm{mt}\right)+((12 / 35) * \mathrm{mb})$;

$k=m * w^{2}$

alpha $=1.67 ; \%$ fudge factor

qin $=(\mathrm{mu} / \mathrm{sqrt}(\mathrm{m} * \mathrm{k})) *(((\mathrm{alpha} * \mathrm{Aq} / \mathrm{d}) *(1+(\mathrm{d} / \mathrm{delta})))+(\mathrm{Ac} / \mathrm{dc})) ; \mathrm{Q}=1 /$ qin;

$\mathrm{dr}=\mathrm{qin} / 2 \%$ Ansys input

$\mathrm{an}=\mathrm{dr} /\left(\mathrm{pi}^{*} \mathrm{f}\right)$;

$\mathrm{c}=\mathrm{dr} * 2 * \operatorname{sqrt}(\mathrm{m} * \mathrm{k}) ; \%$ verification

$\mathrm{nu}=\mathrm{mu} / \mathrm{w}$ 
$\%$ average.m used to extract the maximum value of the optical signal $\% \mathrm{n}$ is the defect free end and a is the defective end optical signal \%Input both these values from the experimental excel sheet

$[p]=\operatorname{peakdetect}(n)^{1}$;

$[q]=\operatorname{peakdetect}(a)$;

$\mathrm{b}=\operatorname{size}(\mathrm{p})$;

$\mathrm{c}=\operatorname{size}(\mathrm{q})$; for $\mathrm{i}=1: \mathrm{b}(1) \operatorname{maxn}(\mathrm{i})=\mathrm{n}(\mathrm{p}(\mathrm{i}))$;

end for $\mathrm{i}=1: \mathrm{c}(1) \operatorname{maxa}(\mathrm{i})=\mathrm{a}(\mathrm{q}(\mathrm{i}))$;

end

$\operatorname{valn}=\operatorname{mean}(\operatorname{maxn})$

vala $=\operatorname{mean}(\operatorname{maxa})$

$\operatorname{diff}=$ valn-vala

\footnotetext{
${ }^{1}$ mathworks.com
} 


\section{Bibliography}

[1] Concept of viscosity, Internet.

[2] Introduction to mems, mems processing, Tech. report, Class notes published online.

[3] R. D. Blanton A. Kolpekwar and C.Kellen, Mems fault model generation using caramel, Proceedings International Test Conference (1998), 557-66.

[4] Scott A.Rittenhouse, Diagnosis of operational changes in microelectromechanical systems via fault detection, Master's thesis, West Virginia University, 2004.

[5] Klaus-Jurgen Bathe, Finite element procedures, Prentice-Hall Inc, 1996.

[6] R.D Blanton and N.Deb, Multi-modal built-in self-test for symmetric microsystems, Proceedings. 22nd IEEE VLSI Test Symposium (2004), 139-147.

[7] Arthur P Boresi and Richard J Schmidt, Advanced mechanics of materials, sixth ed., Wiley, WSC.

[8] B.Stark, Material properties.

[9] Aris Christou, Materials and reliability issues in mems and microsystems, 1993.

[10] A.Kolpekwar C.Kellen and R.D.Blanton, Caramel: Contamination and reliability analysis of microelectromechanical layout, Journal of MicroElectroMechanical Systems 8 (1999), no. 3, 309-317.

[11] Ramaswamy Mahavedevan Mark Stondfield David Koester, Allen Cowen and Buzz Hardy. 
[12] Jeremy Dawson, Integrated through-wafer optical monitoring of mems for closed-loop control, Ph.D. thesis, West Virginia University, 2002.

[13] Deb.N and R. D.Blanton, Analysis of failure sources in surface-micromachined mems, Proceedings of International Test Conference (2000), 739-49.

[14] Gary K Fedder, Simulation of microelectromechanical systems, Ph.D. thesis, University of California at Berkeley, 1994.

[15] Mohammed Gad-El-Hak (ed.), The mems handbook, CRC Press, 2002.

[16] Kazunori Hoshino and Isao Shimoyama, An elastic thin-film microlens array with a pneumatic actuator, 14th IEEE Intl Conference on MEMS (2001), 21-25.

[17] ANSYS Incorporation, Ansys theory reference, Reference Manual.

[18] D. Katzer J. Bagdahn and Halle (Saale) Germany M. Petzold, Fraunhofer Institute for Mechanics of Materials, Strength and reliability of silicon based mems for automotive and medical applications, (2000), 1-8.

[19] J. Chen P. F. Famouri J. M. Dawson, L. Wang and L. A. Hornak, Mems feedback control using through-wafer optical device monitoring, Proceedings of SPIE 4178 (2000), 221-231.

[20] Tao Jiang and R. D.Blanton, Particulate failures for surface-micromachined mems, Proceedings of IEEE Computer Society International Test Conference (1999), 329-337.

[21] Swetha Katipalli, Analysis and optical monitoring of lateral comb resonator under variation of mass and spring geometry, Master's thesis, West Virginia University, 2004.

[22] A. Kolpekwar and R. D. Blanton, Development of mems testing methodology, IEEE International Test Conference (1997), 923-931.

[23] B.; Collard D.; Buchaillot L Millet.O; Agache, V.; Legrand, Modeling offailure mechanisms for optimized mems cad: Design, fabrication and characterization of in-situ test benches, IEEE International Solid-State Sensors and Actuators Conference (2003), 1578-81. 
[24] M.M.Teymoori and E.Abbaspour-Sani, A novel electrostatic micromachined pump for drug delivery systems, Semiconductor Electronics Proceedings, IEEE (2002), 105-109.

[25] H.; Badawy W.; Moussa M. Moussa, W.A; Ahmed, Investigating the reliability of electrostatic comb-drive actuators used in microfluidic and space systems using finite element analysis, Canadian Journal of Electrical and Computer Engineering 27 (2002), no. 4, 195-200.

[26] Senol Mutlu and Brian Jensen, Design and simulation of shaped comb fingers for compensation of mechanical restoring force, Proceedings of ASME International Mechanical Engineering Congress and Exposition (2001), 923-931.

[27] T. Mukherjee N. Deb, S.V.Iyer and R. D. Blanton, Mems resonator synthesis for defect reduction, Journal of Modeling and Simulation of Microsystems 2 (2001), no. 1, 11-20.

[28] N.Deb and R. D. Blanton, Built-in self test of cmos-mems accelerometers, International Test Conference (2002), 7-10.

[29] Zhijian Li Quanbo Zou and Litian Liu, Study of methods for measuring mechanical properties of thin films in microelectromechanical systems (mems), Proceedings of 4th International Conference on Solid-State and IC Technology (1995), 488-490.

[30] A.M. Richardson R. Rosing and A.P. Dorey, A fault simulation methodology for mems, Proceedings Design, Automation and Test in Europe Conference and Exhibition 2000 (2000), 476-83.

[31] A.P. Dorey R. Rosing, A.M. Richardson and A.Peyton, Test support strategies for mems, IEE Colloquium Intelligent and Self-Validating Sensors (1999), 7/1-7/6.

[32] C.L. Muhlstein R.K. Nalla R.O. Ritchie, J.J. Kruzic and E.A. Stach, Characteristic dimensions and the micro-mechanisms of fracture and fatigue in nano and bio materials, International Journal of Fracture (2004), 1-15. 
[33] B. Charlot S. Mir and B. Courtois, Extending fault-based testing to microelectromechanical systems, Journal of Electronic testing: Theory and Applications 16 (2000), no. 3, 277-288.

[34] Stephen D Senturia, Microsystem design.

[35] J.; Reber J.; Lott J.; Cowan W.and Vandelli N Starman Jr., L.; Busbee, Stress measurement in mems devices, International Conference on Modeling and Simulation of Microsystems MSM 2001 (2001), 398-401.

[36] M. Selim nl Bennett B. Goldberg V. T. Srikar, Anna K. Swan and S. Mark Spearing, Micro-raman measurement of bending stresses in micromachined silicon flexures, Journal of MicroElectroMechanical Systems 12 (2003), no. 6, 779-787.

[37] L. K. Warne W. A. Johnson, Electrophysics of micromechanical comb actuators, Journal of Microelectromechanical Systems 4 (1995), no. 1, 49-59.

[38] Limin Wang, Modeling and real-time feedback control of mems device, Ph.D. thesis, West Virginia University, 2004.

[39] Bin Yuan William N. Sharpe, Jr. and Ranji Vaidyanathan, Measurements of young's modulus, poisson's ratio and tensile strength of polysilicon, Proceedings of the Tenth IEEE International Workshop on Microelectromechanical Systems (1997), 424-429.

[40] M. T. Saif X. T. Huang and N. C. MacDonald, A micromotion amplifier, Proceedings of Ninth International Workshop on Micro Electromechanical Systems (1996), 424-428.

[41] Robert Yates and Ben Salem, Microsystems - an enabling technology for virtual reality, IEE Colloquium on Virtual Reality Personal Mobile and Practical Applications (1998), 2/1-2/4.

[42] Xia Zhang and William C Tang, Viscous air damping in laterally driven microsystems, IEEE Micro electro mechanical Systems workshop (1994), 199-204. 